\title{
Interlaboratory Analytical Comparison Study of Fatty Acid Concentrations in Human Serum- Results for Exercise 03: QA16FASER03
}

\author{
Michele M. Schantz \\ This publication is available free of charge from: \\ http://dx.doi.org/10.6028/NIST.IR.8146
}




\title{
Interlaboratory Analytical Comparison Study of Fatty Acid Concentrations in Human Serum- Results for Exercise 03: QA16FASER03
}

\author{
Michele M. Schantz \\ Chemical Sciences Division \\ Material Measurement Laboratory
}

This publication is available free of charge from:

http://dx.doi.org/10.6028/NIST.IR.8146

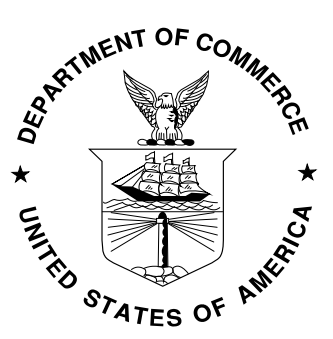

U.S. Department of Commerce Penny Pritzker, Secretary

National Institute of Standards and Technology Willie May, Under Secretary of Commerce for Standards and Technology and Director 


\section{ABSTRACT}

The National Institute of Standards and Technology (NIST), at the request of the National Institute of Health's (NIH's) Office of Dietary Supplements (ODS) and in conjunction with the Centers for Disease Control and Prevention (CDC), initiated a third interlaboratory analytical comparison study of fatty acid concentrations in human serum in 2016. Three unknown serum samples were distributed along with Standard Reference Material (SRM) 1950 Metabolites in Frozen Human Plasma as the control sample. This is a performance-based program so participating laboratories were requested to use the analytical procedures that they typically use in their laboratories for these analyses and report data for those fatty acids that they typically quantify. The results from the third exercise are reported along with a summary of the analytical methods used by each laboratory. 


\section{Background}

The National Institute of Standards and Technology (NIST) currently offers three quality assurance programs (QAPs) for the determination of micronutrients, vitamin D metabolites, and fatty acids in serum and plasma matrices, the Micronutrients Measurement QAP (MMQAP), the Vitamin D QAP (VitDQAP), and the Fatty Acid QAP (FAQAP), respectively. Given the similarity in the operations of these programs, NIST has consolidated the three programs into one larger program, the NIST Clinical Quality Assurance Program, or ClinQAP (http://www.nist.gov/mml/csd/clinqap.cfm). The primary goals of the ClinQAP are to support the comparability of clinical measurements through the MMQAP, VitDQAP, and the FAQAP and to monitor and support the emerging measurement needs of the clinical community.

The first exercise of the FAQAP was conducted in 2012 with 11 laboratories returning data for the content of individual fatty acids in Standard Reference Material (SRM) 2378 Fatty Acids in Frozen Human Serum (a candidate material at that time) along with SRM 1950 Metabolites in Frozen Human Plasma which was distributed as the control sample. Candidate SRM 2378 consists of three serum materials collected from: donors who have not taken fish or flaxseed oil supplements for one month prior to collection; donors who have taken flaxseed oil supplements for a minimum of one month prior to collection; and donors who have taken fish oil supplements for a minimum of one month prior to collection. SRM 1950 is designed to represent "normal" human plasma. Plasma was obtained from 100 individuals (equal number of men and women in a narrow age range (40 to 50 years) who had undergone an overnight fast prior to blood draw. The results from the first exercise were published in NIST Internal Report (NISTIR) 7953. [1]

The second exercise of the FAQAP was conducted in 2015 with 14 laboratories returning data for the content of individual fatty acids in three serum samples, that had been originally collected for use in the MMQAP, and in each of the three levels of SRM 2378 (as control samples). The results from the second exercise were published in NISTIR 8086 [2].

\section{Overview of Exercise 03: QA16FASER03}

For the third FAQAP exercise, the intercomparison materials were three serum samples previously used in the VitDQAP studies. [3] The samples used were:

$$
\begin{array}{ll}
\text { SRM 972a Level } 2 \text { relabeled as } & \text { FAQAP Unk 004 (black label) } \\
\text { VitDQAP Sample I relabeled as } & \text { FAQAP Unk 005 (red label) } \\
\text { VitDQAP Sample II relabeled as } & \text { FAQAP Unk 006 (green label) }
\end{array}
$$

In addition, participants were requested to run SRM 1950 as the control material. The laboratories were provided with three vials of each unknown material, each vial contained approximately $1 \mathrm{~mL}$ of serum, and were requested to do triplicate measurements of each unknown sample using their laboratory's and/or program's analytical protocols for the concentrations of the fatty acids currently being determined in their laboratory. In addition, they 
were requested to analyze one subsample of the control material, SRM 1950. A target list of fatty acids was provided; however, participants did not need to quantify all of these compounds and could add additional compounds when reporting data. They were requested to report results, using three significant figures, in units of either $\mu \mathrm{g} / \mathrm{g}$ or $\mu \mathrm{mol} / \mathrm{L}(\mu \mathrm{M})$ and to provide brief descriptions of their cleanup and analytical procedures.

\section{Reported Results}

Thirteen laboratories returned data and were assigned numerical identification codes in order of receipt of data with the exception of the NIST laboratory, which is Lab 1 in this exercise. The fatty acids included in the study are listed in Table 1 with the suggested list of fatty acids at the top of the table and the additional fatty acids reported listed below. For the total (esterified and free) fatty acids, the laboratory mean values, standard deviations, and relative standard deviations are shown in Tables 2, 4, and 6 in $\mu \mathrm{g} / \mathrm{g}$ for unknowns 004, 005, and 006, respectively, and in Tables 3, 5, and 7 in $\mu \mathrm{M}$ for unknowns 004, 005, and 006, respectively. The laboratory values reported for the control sample, SRM 1950, are summarized in Tables 8 and 9 in $\mu \mathrm{g} / \mathrm{g}$ and in $\mu \mathrm{M}$, respectively. The summary statistics (median, number of laboratories reporting, and lowest and highest value reported) are given in Table 10 for the total fatty acids.

This is the first study in which the laboratories were also given the option of reporting data for the free fatty acids in the serum samples. Only one laboratory (Lab 12) did so, however. Their data for the free fatty acids are shown in Table 11, and the \% of free to total fatty acid based only on that laboratory's data are shown in Table 12.

Summaries of the methods used and notes submitted by each laboratory are in Appendix A, and charts of the mean numerical results reported by each laboratory for each analyte in the exercise materials are in Appendix B.

\section{Discussion}

NIST contacted over 40 laboratories in early 2016 regarding participation in the third interlaboratory analytical comparison study of fatty acid concentrations in human serum. Of the laboratories contacted, 15, including NIST, replied with interest in participating in this study. As stated above, laboratories were also given the option of reporting free fatty acids along with total fatty acids for the samples received. Of the 15 laboratories requesting samples, four (4) said that they were interested in quantifying the free fatty acids in addition to the total fatty acids and two

(2) were interested in quantifying the free fatty acids. Only one of those laboratories, designated as Lab 12 in this study, reported data for the free fatty acids.

Samples were sent on dry ice between March 14 and 21, 2016. The deadline for submission of data to NIST was June 1, 2016. All but two laboratories returned data by June 30, 2016. One of the remaining laboratories reported that they would not be sending in data because they had 
problems with the hydrolysis step resulting in low results on the control material. The last laboratory did not respond to requests for a status report on their analyses.

After submitting data, one of the laboratories (designated as Lab 11 in this study) asked that their data be removed from the data summary. Their results were 5 to 10 times higher than expected based on the SRM 1950 values. They stated that their method is semi-quantitative, reported as normalized area percents. They have had success with the method for foods but speculate that the residue mass from the serum and plasma samples is composed of more than fatty acids thus overestimating the concentrations of each fatty acid.

Another laboratory (designated as Lab 13 in this study) provided a recalculated set of data on July 12. The recalculated data set has been incorporated into this report. The laboratory did not report what the recalculation involved.

An alphabetical list of laboratories that returned data is given in Appendix C. Note that the numerical codes in the following tables and figures are based on the receipt of the data and therefore do not follow the order in Appendix C.

Laboratories were requested to submit data for the fatty acids that they typically monitor in similar samples (Table 1) for three subsamples of each unknown sample received and one subsample of SRM 1950 in units of either $\mu \mathrm{g} / \mathrm{g}$ or $\mu \mathrm{M}$ along with the density of each sample. Summaries of the results submitted by each laboratory are presented in Tables 2 through 7 as the means, standard deviations, and relative standard deviations for the three values submitted for each unknown sample in both $\mu \mathrm{g} / \mathrm{g}$ and $\mu \mathrm{M}$. Three (3) laboratories reported the data in $\mu \mathrm{g} / \mathrm{g}$, and nine (9) laboratories reported the data in $\mu \mathrm{M}$. The conversions between the units reported and the alternate units were calculated using the densities provided by each laboratory except for labs 1, 2, 5, 7, and 13. These laboratories did not report a density so the density was assumed to be $1 \mathrm{~g} / \mathrm{mL}$ for the conversion. The densities reported by the remaining laboratories are shown in the tables and range from $0.954 \mathrm{~g} / \mathrm{mL}$ to $1.09 \mathrm{~g} / \mathrm{mL}$. Summaries of the results from each participant for the analysis of one subsample of SRM 1950 are presented in tables 8 and 9 in $\mu \mathrm{g} / \mathrm{g}$ and $\mu \mathrm{M}$, respectively, along with the certified and reference values for the fatty acids.

As shown in Tables 2 through 7, the relative standard deviations (RSDs) for each participating laboratory for the analyses of three subsamples for each unknown serum sample are generally < $10 \%$. Lab 13 had a few analytes, particularly in unknown 004, with higher RSDs as well as high RSDs (> 90 \%) for C22:1n9 in unknown 005 and unknown 006. The higher RSDs for C22:1n9 came from reporting one or more 0 values for the subsamples.

Several laboratories reported data for additional fatty acids. These fatty acids are included at the bottom of Table 1 and summarized at the bottom of Tables 2 through 9.

The data in terms of $\mu \mathrm{M}$ are plotted by analyte in each material in Appendix B. Each data point is the mean and associated standard deviation of the data reported by the laboratory of the indicated analyte in the indicated sample. The medians calculated from the data received for the fatty acid in each unknown sample are summarized in Table 10 and included on each chart for 
the unknown samples. The charts for SRM 1950 include the certified or reference values for comparison purposes.

The relative expanded uncertainty for the medians ranged from $9 \%$ to $66 \%$ for unknown 004, from $7 \%$ to $>300 \%$ for unknown 005 , and $<1 \%$ to $>200 \%$ for unknown 006 . The high relative uncertainties for C22:1n9 in unknown 005 and 006 are due to a limited number of laboratories reporting data $(\mathrm{n}=4)$, and one of those laboratories ( $\mathrm{Lab} 13)$ appearing to be an outlier. As mentioned above, this is also the compound for which Lab 13 reported several 0 values resulting in high RSDs for their data. In general, the agreement among the laboratories was best in all of the samples for those fatty acids at highest concentrations, particularly C16:0, C18:0, C18:1n9, and C18:2n6.

Examples of the comparison of the median values and associated uncertainties are shown below for 10 of the fatty acids quantified in this study.

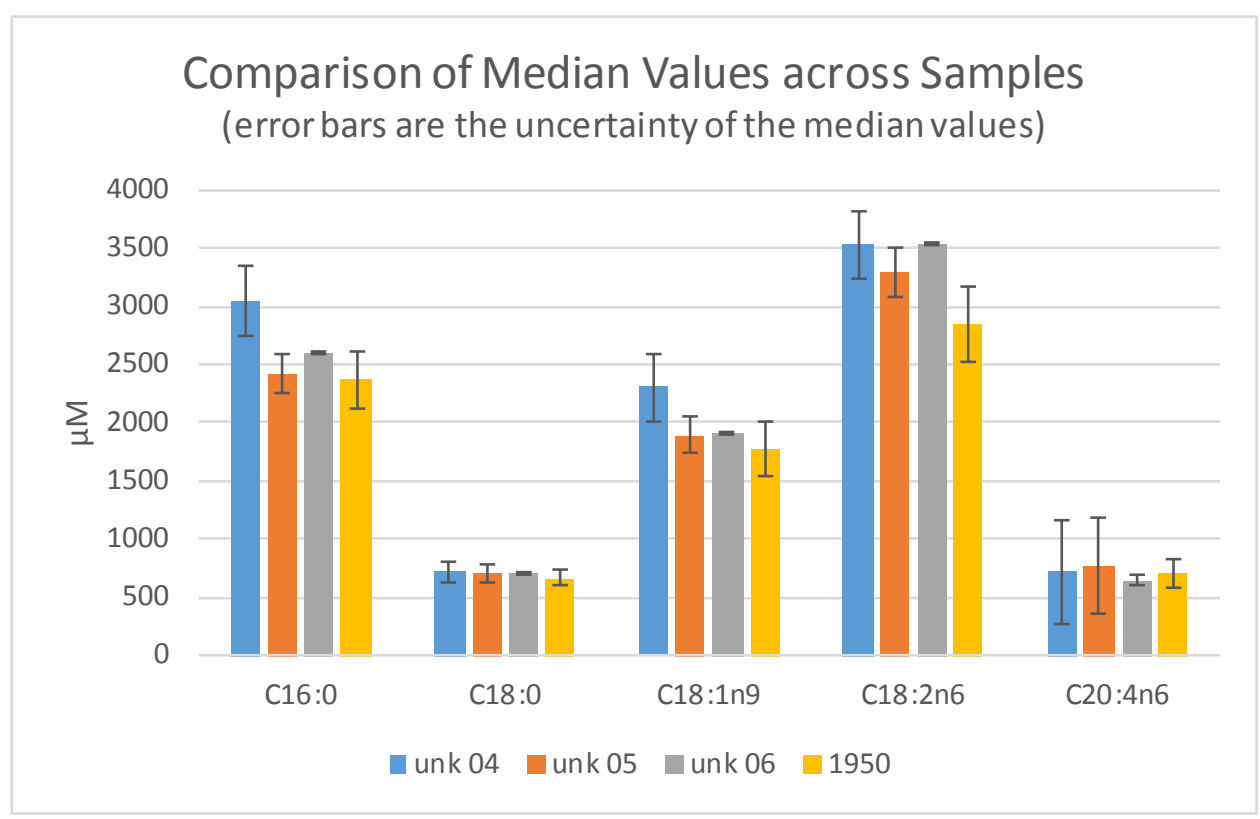




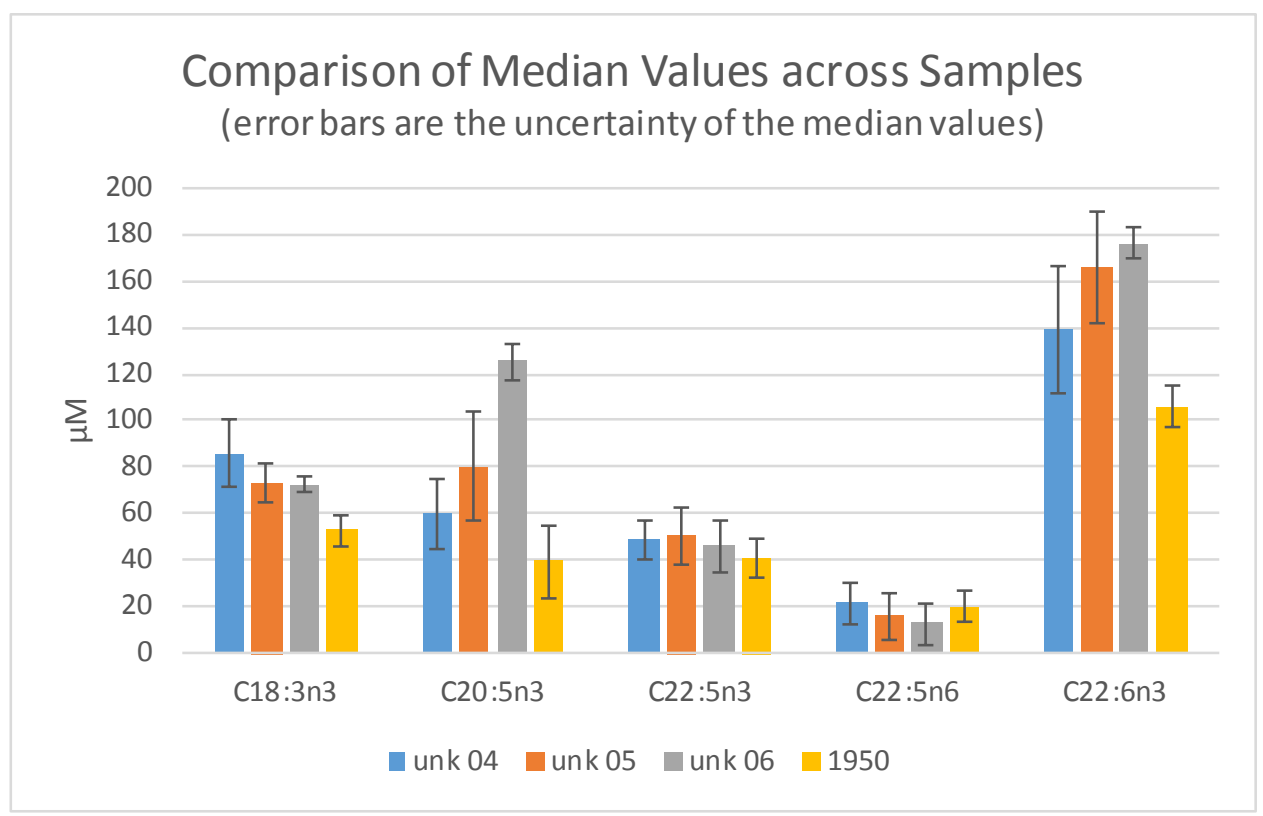

The concentrations of the individual fatty acids in the four (4) samples quantified in this study were similar with the uncertainty of the median values overlapping for most of the fatty acids with the exception of C20:5n3 being highest in unknown 006. It is interesting to note that the agreement among the laboratories was best for the majority of the fatty acids in unknown 006 .

In the past exercises [1,2], there were some trends across all fatty acids in the samples with a particular laboratory reporting high data or low data. In this exercise, that does not appear to be the case. The laboratories reporting the highest or lowest data tend to vary from fatty acid to fatty acid within the samples, however, tend to be consistent across samples. Using C20:4n6 as an example, Lab 2 reported the lowest values for unknown 004, unknown 005, and SRM 1950 and the second lowest value for unknown 006; whereas Lab 7 reported the highest value for all of the samples. These results could be obtained by incomplete extraction (low), coelution with other fatty acids or matrix components on the analytical column used (high), misidentification of the chromatographic peaks (low or high), or from some other method variations such as inaccurate calibration.

The methods used by each laboratory are summarized in Appendix A with the procedures for the total fatty acids in Table A-1, Lab 12's procedures for the free fatty acids in Table A-2, the calibration information in Table A-3, and notes submitted by laboratories in Table A-4. For the laboratories reporting an hydrolysis method, they used both acid and base, and the extraction methods were typically liquid-liquid. Most laboratories used gas chromatography (GC) with either flame ionization detection (FID) or mass spectrometry (MS) following a derivatization. The derivatizing reagants used by each laboratory are summarized in Appendix A. The exception being lab 7, which used a liquid chromatography (LC)/ MS/MS method. They, however, reported data for only three fatty acids, C20:4n6, C20:5n3, and C22:6n3. 
For the GC analyses, the columns used ranged in polarity from relatively non-polar (5\% phenyl methylpolysiloxane phase) to relatively polar (cyanopropyl phase). For the calibration information (Table A-3), labs 1, 2, 7, 8, 9, 10 used a linear regression, labs 3, 4, and 12 used a quadratic regression, and lab 6 used a mix of linear and quadratic regression tailored by analyte. Lab 5 only reported the limits of their calibration range, and labs 11 and 13 did not report calibration information. Combining the method information with the trends in the data reported does not lead to obvious explanations of the variations noted in the data submitted by individual laboratories.

Lab 12 reported data for the free fatty acids (Table 11) in addition to the total fatty acids. Using only lab 12's data, the relative amount of free to total for each fatty acid in the four samples has been calculated and is summarized in Table 12 . The free fatty acids are from $<1 \%$ to $34 \%$ of the total fatty acids with most in the $2 \%$ to $10 \%$ range. Hopefully, more laboratories will report data for free fatty acids in future intercomparison exercises.

The data from this exercise can be used to assess the comparability across a limited number of laboratories (see Appendix C). The data do not indicate specific method biases but do indicate the need for such intercomparisons and for the use of control materials to increase the comparability of data across laboratories.

\section{Acknowledgments}

The financial support for this intercomparison exercise was provided by the National Institutes of Health (NIH), Office of Dietary Supplements (ODS). Technical coordination was provided by Joseph M. Betz of NIH-ODS.

The time and effort of the analysts and management of the participating laboratories are gratefully acknowledged.

\section{Disclaimer}

Certain commercial equipment, instruments, or materials are identified in this report to specify adequately the experimental procedure. Such identification does not imply recommendation or endorsement by the National Institute of Standards and Technology, nor does it imply that the materials or equipment identified are the best available for the purpose.

\section{References}

1. Schantz M.M. Powers C,D., and Schleicher, R Interlaboratory Analytical Comparison Study of Total Fatty Acid Concentrations in Human Serum: Results for Exercise 01: QA12FASER01, NISTIR 7953, Gaithersburg, MD (2013). 
2. Schantz, M.M. Interlaboratory Analytical Comparison Study of Fatty Acid Concentrations in Human Serum: Results for Exercise 02: QA15FASER02, NISTIR 8086, Gaithersburg, MD (2015).

3. Bedner, M., Lippa, K.A., Tai, S. S.-C. An assessment of 25-hydroxyvitamin D measurements in comparability studies conducted by the Viatamin D Metabolites Quality Assurance Program, Clinica Chimica Acta 426 (2013) 6-11. 


\begin{tabular}{|c|c|c|c|}
\hline \multicolumn{2}{|c|}{ Table 1. Fatty Acids Included in Study } & \multirow[b]{2}{*}{$\mathrm{mol} \mathrm{wt}(\mathrm{g} / \mathrm{mol})$} & \\
\hline Code & Common Name & & \\
\hline C14:0 & Myristic acid & 228.38 & \\
\hline C14:1n5 & Myristoleic acid & 226.38 & \\
\hline C16:0 & Palmitic acid & 256.43 & \\
\hline C16:1n7 & Palmitoleic acid & 254.43 & \\
\hline C18:0 & Stearic acid & 284.48 & \\
\hline C18:1n7 & cis-Vaccenic acid & 282.48 & \\
\hline C18:1n9 & Oleic acid & 282.48 & \\
\hline C18:2n6 & Linoleic acid & 280.48 & \\
\hline C18:3n3 & alpha-Linolenic acid & 278.48 & \\
\hline C18:3n6 & gamma-Linolenic acid & 278.48 & \\
\hline C20:0 & Arachidic acid & 312.54 & \\
\hline C20:1n9 & 11-Eicosenoic acid & 310.54 & \\
\hline C20:2n6 & 11,14-Eicosadienoic acid & 308.53 & \\
\hline C20:3n6 & homo-gamma-Linolenic acid & 306.53 & \\
\hline C20:4n6 & Arachidonic acid & 304.52 & \\
\hline$C 20: 5 n 3$ & Eicosapentaenoic acid & 302.52 & \\
\hline $\mathrm{C} 22: 0$ & Docosanoic acid & 340.59 & \\
\hline C22:1n9 & Docosenoic acid & 338.59 & \\
\hline $\mathrm{C} 22: 4 \mathrm{n} 6$ & Docosatetraenoic acid & 332.57 & \\
\hline$C 22: 5 n 3$ & Docosapentaenoic acid & 330.57 & \\
\hline C22:5n6 & Docosapentaenoic acid & 330.57 & \\
\hline C22:6n3 & Docosahexaenoic acid & 328.57 & \\
\hline C24:0 & Lignoceric acid & 368.64 & \\
\hline C24:1n9 & Nervonic acid & 366.63 & \\
\hline \multicolumn{4}{|c|}{ List any additional information such as known coelutions or results for additional fatty acids below: } \\
\hline C8:0 & Caprylic acid & 144.26 & \\
\hline C10:0 & Decanoic acid & 172.3 & \\
\hline C10:1n1 & 9-Decenoic acid & 170.3 & \\
\hline C12:0 & Lauric acid & 200.34 & \\
\hline C12:1n1 & 11-Dodecenoic acid & 198.34 & \\
\hline C12:1n7 & cis-5-dodecenoic & 198.34 & \\
\hline C14:2 & & 224.38 & \\
\hline C15:0 & Pentadecanoic acid & 242.42 & \\
\hline C16:1n7t & Palmitelaidic acid & 254.43 & \\
\hline C16:1n9 & & 254.43 & \\
\hline C16:2 & & 252.43 & \\
\hline C17:0 & Margaric acid & 270.46 & \\
\hline C17:1n9 & 10-Heptadecenoic acid & 268.46 & \\
\hline $9 c, 11 t-C 18: 2 n 6$ & Rumenic acid & 280.48 & \\
\hline C18:4n3 & Stearidonic acid & 276.48 & \\
\hline C19:0 & Nonadecanoic acid & 298.54 & \\
\hline C20:3n3 & 11,14,17-Eicosatrienoic acid & 306.53 & \\
\hline C20:3n9 & 5,8,11-Eicosatrienoic acid (Mead acid) & 306.53 & \\
\hline C20:4n3 & Arachidonic acid (Omega 3) & 304.53 & \\
\hline C21:0 & Heneicosanoic acid & 326.58 & \\
\hline $\mathrm{C} 22: 2 \mathrm{n} 6$ & Docosadienoic acid & 336.59 & \\
\hline C23:0 & Tricosanoic acid & 354.62 & \\
\hline $\mathrm{C} 26: 0$ & Hexacosanoic acid & 396.68 & \\
\hline $\mathrm{C} 26: 1$ & & 394.68 & \\
\hline $\mathrm{PhA}$ & Phytanic acid & 312.54 & \\
\hline PrA & Pristanic acid & 298.5 & \\
\hline
\end{tabular}




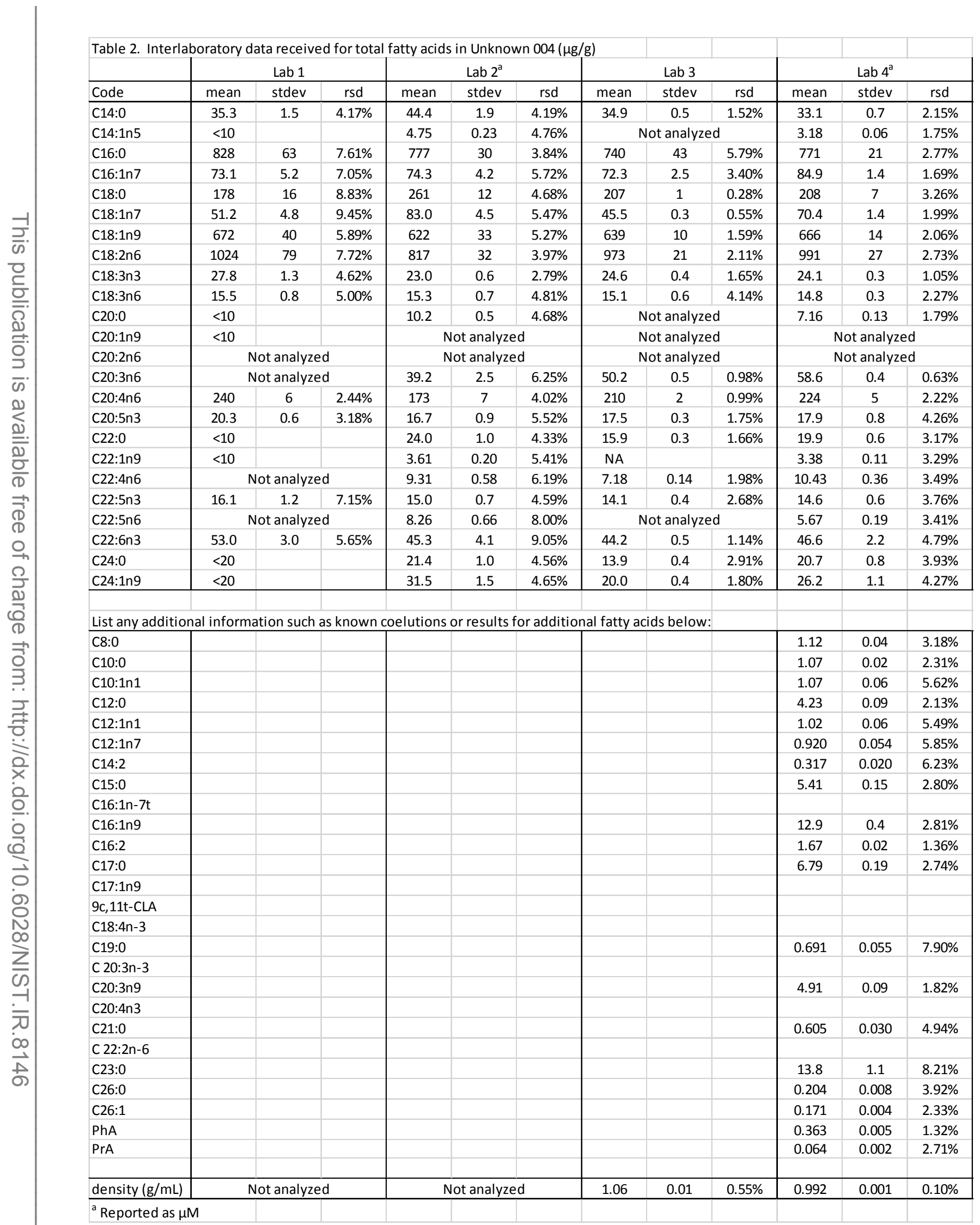




\begin{tabular}{|c|c|c|c|c|c|c|c|c|c|c|c|c|}
\hline \multicolumn{10}{|c|}{ Table 2 (cont.). Interlaboratory data received for total fatty acids in Unknown $004(\mu \mathrm{g} / \mathrm{g}$ ) } & \multirow{2}{*}{\multicolumn{3}{|c|}{ Lab 8}} \\
\hline & \multicolumn{3}{|c|}{ Lab $5^{a}$} & \multicolumn{3}{|c|}{$\operatorname{Lab} 6^{\mathrm{a}}$} & \multicolumn{3}{|c|}{$\operatorname{Lab} 7^{\mathrm{a}}$} & & & \\
\hline C14:0 & 38.7 & 1.1 & $2.91 \%$ & 38.6 & 1.0 & $2.54 \%$ & \multicolumn{3}{|c|}{ Not analyzed } & 46.2 & 3.9 & $8.33 \%$ \\
\hline C14:1n5 & & & & 2.52 & 0.04 & $1.67 \%$ & \multicolumn{3}{|c|}{ Not analyzed } & \multicolumn{3}{|c|}{ Not analyzed } \\
\hline C16:0 & 780 & 17 & $2.15 \%$ & 845 & 19 & $2.23 \%$ & \multicolumn{3}{|c|}{ Not analyzed } & 1050 & 73 & $6.93 \%$ \\
\hline C18:1n7 & & & & 44.2 & 2.3 & $5.29 \%$ & \multicolumn{3}{|c|}{ Not analyzed } & \multicolumn{3}{|c|}{ Not analyzed } \\
\hline C18:1n9 & 625 & 13 & $2.02 \%$ & 677 & 28 & $4.10 \%$ & \multicolumn{3}{|c|}{ Not analyzed } & 861 & 59 & $6.85 \%$ \\
\hline C18:2n6 & 977 & 19 & $1.94 \%$ & 1029 & 39 & $3.78 \%$ & & t analy & & 1084 & 78 & $7.21 \%$ \\
\hline C18:3n3 & 23.2 & 0.5 & $1.98 \%$ & 28.8 & 1.2 & $4.09 \%$ & & t analy & & 35.9 & 3.0 & $8.31 \%$ \\
\hline C18:3n6 & & & & 16.3 & 0.8 & $4.98 \%$ & & t analy & & & t analy & \\
\hline C2O:0 & & & & 6.60 & 0.18 & $2.79 \%$ & & t analy & & & $t$ analy & \\
\hline$C 20: 5 n 3$ & 15.4 & 0.5 & $3.07 \%$ & 19.4 & 0.1 & $0.36 \%$ & 27.4 & 1.7 & $6.07 \%$ & 28.7 & 3.6 & $12.44 \%$ \\
\hline C22:0 & & & & 22.6 & 0.1 & $0.37 \%$ & & t analy & & & $t$ analy & \\
\hline C22:1n9 & & & & & & & & $t$ analy & & & $t$ analy & \\
\hline C22:4n6 & & & & 8.93 & 0.21 & $2.36 \%$ & & t analy & & 11.2 & 1.2 & $10.43 \%$ \\
\hline$C 22: 5 n 3$ & 18.5 & 1.2 & $6.28 \%$ & 16.8 & 0.8 & $4.81 \%$ & & t analy & & 18.5 & 1.2 & $6.61 \%$ \\
\hline C22:5n6 & & & & 7.32 & 0.08 & $1.05 \%$ & & t analy & & & $t$ analy & \\
\hline$C 22: 6 n 3$ & 37.6 & 1.8 & $4.84 \%$ & 53.4 & 2.7 & $5.10 \%$ & 70.2 & 0.8 & $1.18 \%$ & 18.5 & 1.2 & $6.61 \%$ \\
\hline C24:0 & & & & 20.3 & 0.5 & $2.51 \%$ & & $t$ analy & & & $t$ analy & \\
\hline C24:1n9 & & & & 31.4 & 0.8 & $2.50 \%$ & & $t$ analy & & & $t$ analy & \\
\hline & & & & & & & & & & & & \\
\hline List any additio & al inform & on such & s known & elution & results & r additic & al fatty a & s belo & & & & \\
\hline C16:1n-7t & & & & & & & & & & & & \\
\hline C16:1n9 & & & & & & & & & & & & \\
\hline C16:2 & & & & & & & & & & & & \\
\hline C17:0 & & & & 8.22 & 0.16 & $1.89 \%$ & & & & & & \\
\hline C17:1n9 & & & & & & & & & & & & \\
\hline $9 c, 11 \mathrm{t}-\mathrm{CLA}$ & & & & & & & & & & & & \\
\hline C18:4n-3 & & & & 1.10 & 0.05 & $4.30 \%$ & & & & & & \\
\hline C19:0 & & & & & & & & & & & & \\
\hline C $20: 3 n-3$ & & & & & & & & & & & & \\
\hline$C 20: 3 n 9$ & & & & 2.34 & 0.09 & $4.02 \%$ & & & & & & \\
\hline$C 20: 4 n 3$ & & & & & & & & & & & & \\
\hline C21:0 & & & & & & & & & & & & \\
\hline C $22: 2 n-6$ & & & & & & & & & & & & \\
\hline C23:0 & & & & 10.3 & 0.2 & $1.89 \%$ & & & & & & \\
\hline C26:0 & & & & & & & & & & & & \\
\hline $\mathrm{C} 26: 1$ & & & & & & & & & & & & \\
\hline PhA & & & & & & & & & & & & \\
\hline PrA & & & & & & & & & & & & \\
\hline & & & & & & & & & & & & \\
\hline density $(\mathrm{g} / \mathrm{mL})$ & & t analyz & & 0.963 & 0.003 & $0.32 \%$ & & $t$ analy & & 1.03 & 0.01 & $1.12 \%$ \\
\hline${ }^{\mathrm{a}}$ Reported as $\mu$ & & & & & & & & & & & & \\
\hline
\end{tabular}




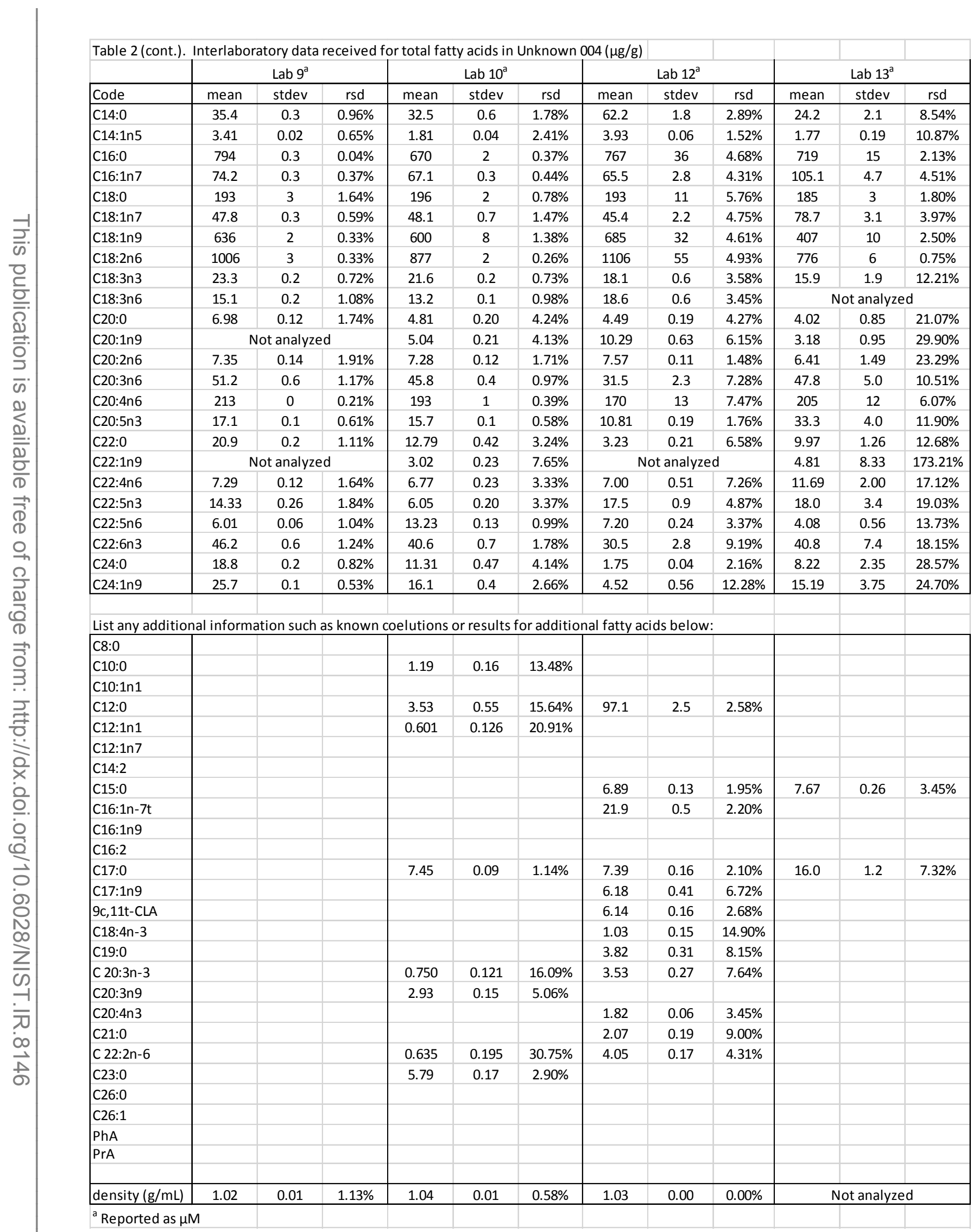




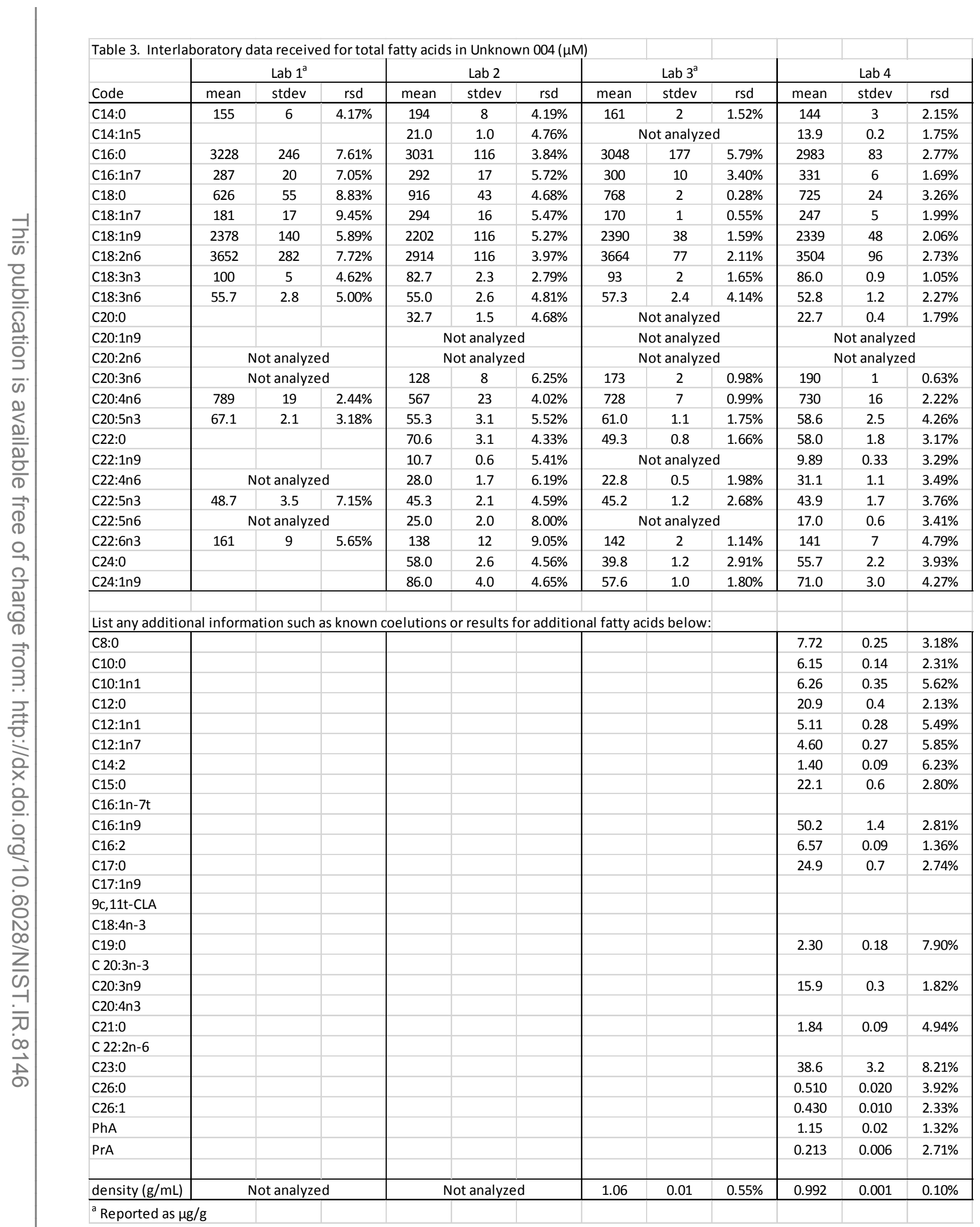




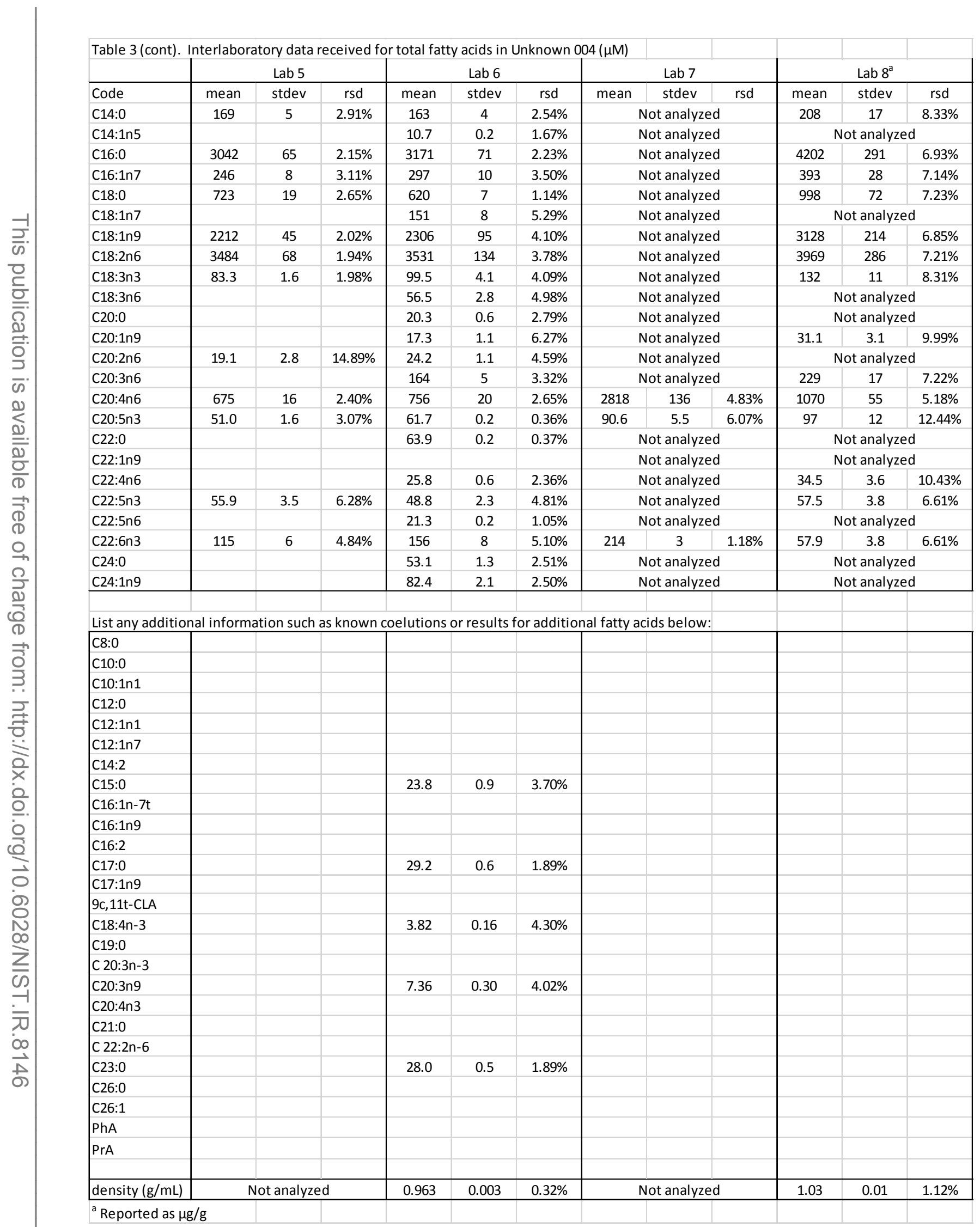




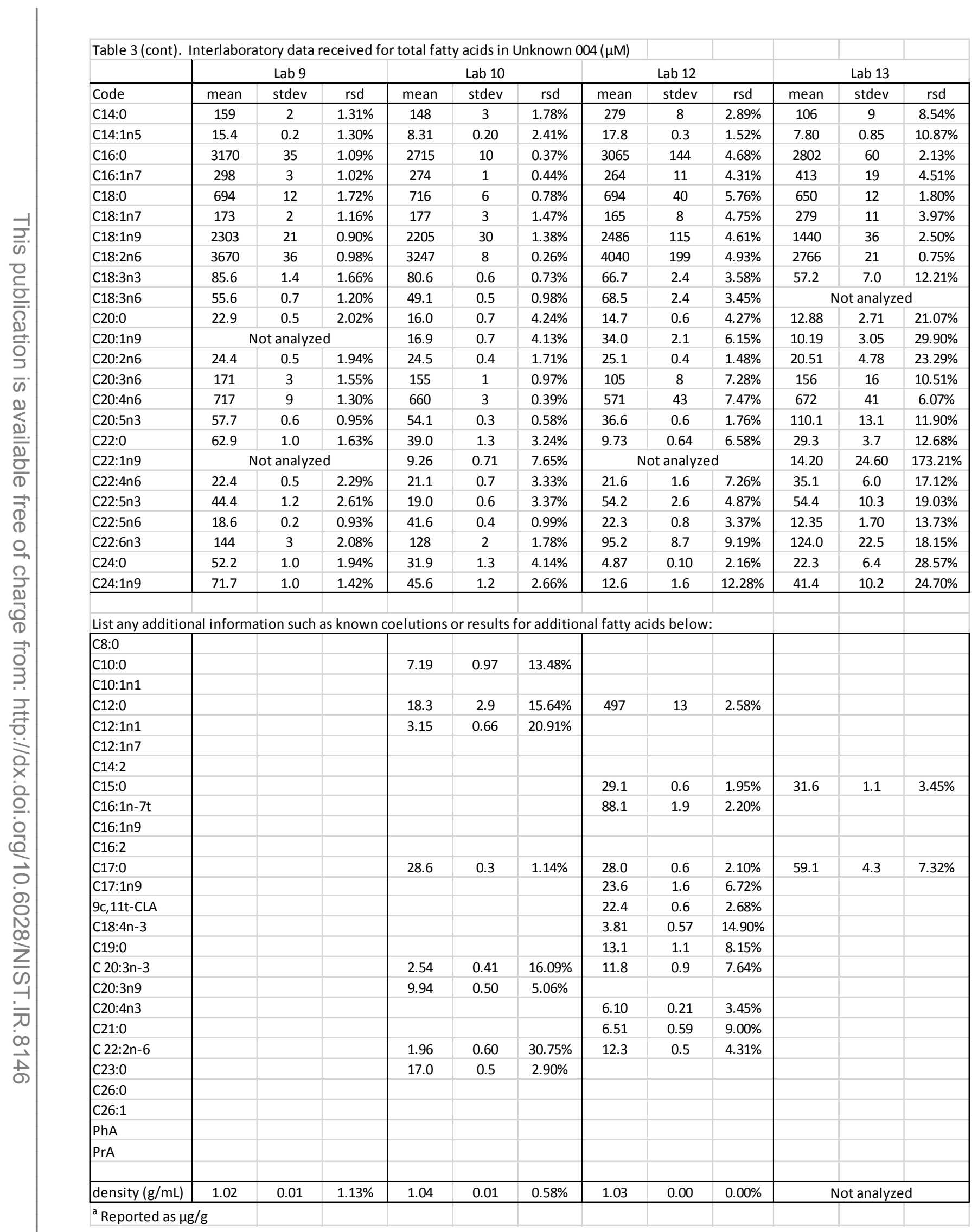




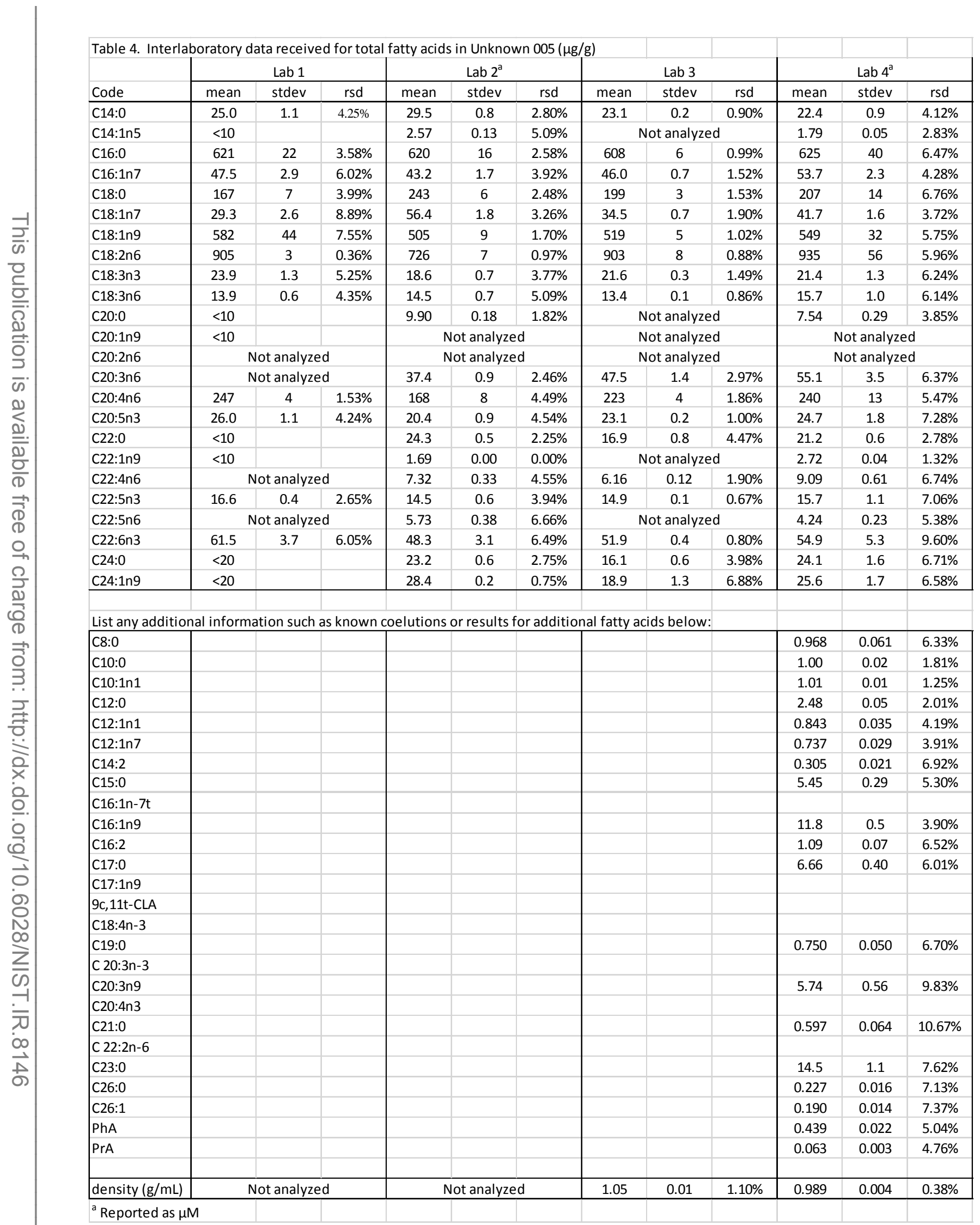




\begin{tabular}{|c|c|c|c|c|c|c|c|c|c|c|c|c|}
\hline \multicolumn{10}{|c|}{ Table 4 (cont). Interlaboratory data received for total fatty acids in Unknown $005(\mu \mathrm{g} / \mathrm{g})$} & \multirow{2}{*}{\multicolumn{3}{|c|}{ Lab 8}} \\
\hline & \multicolumn{3}{|c|}{$\operatorname{Lab} 5^{\mathrm{a}}$} & \multicolumn{3}{|c|}{$\operatorname{Lab} 6^{\mathrm{a}}$} & \multicolumn{3}{|c|}{$\operatorname{Lab} 7^{\mathrm{a}}$} & & & \\
\hline C14:0 & 25.4 & 0.3 & $1.01 \%$ & 25.3 & 0.4 & $1.45 \%$ & \multicolumn{3}{|c|}{ Not analyzed } & 24.6 & 0.3 & $1.28 \%$ \\
\hline C14:1n5 & & & & 1.38 & 0.04 & $3.25 \%$ & \multicolumn{3}{|c|}{ Not analyzed } & \multicolumn{3}{|c|}{ Not analyzed } \\
\hline C16:0 & 615 & 2 & $0.32 \%$ & 679 & 7 & $1.07 \%$ & \multicolumn{3}{|c|}{ Not analyzed } & 768 & 10 & $1.31 \%$ \\
\hline C18:1n7 & & & & 34.6 & 0.4 & $1.28 \%$ & \multicolumn{3}{|c|}{ Not analyzed } & \multicolumn{3}{|c|}{ Not analyzed } \\
\hline C18:1n9 & 509 & 2 & $0.34 \%$ & 572 & 0 & $0.08 \%$ & \multicolumn{3}{|c|}{ Not analyzed } & 586 & 4 & $0.70 \%$ \\
\hline C18:2n6 & 912 & 2 & $0.25 \%$ & 992 & 6 & $0.61 \%$ & & t analy & & 918 & 16 & $1.71 \%$ \\
\hline C18:3n3 & 20.2 & 0.1 & $0.54 \%$ & 25.2 & 0.1 & $0.41 \%$ & & t analy & & 26.8 & 0.3 & $1.21 \%$ \\
\hline C18:3n6 & & & & 14.69 & 0.23 & $1.59 \%$ & & t analy & & & t analyz & \\
\hline C20:0 & & & & 6.52 & 0.18 & $2.74 \%$ & & t analy & & & $t$ analyz & \\
\hline$C 20: 5 n 3$ & 21.6 & 0.2 & $0.86 \%$ & 26.09 & 0.36 & $1.40 \%$ & 25.0 & 0.9 & $3.58 \%$ & 32.3 & 0.5 & $1.54 \%$ \\
\hline C22:0 & & & & 23.9 & 0.2 & $0.87 \%$ & & t analy & & & $t$ analyz & \\
\hline C22:1n9 & & & & & & & & t analy & & & $t$ analyz & \\
\hline C22:4n6 & & & & 7.26 & 0.26 & $3.64 \%$ & & t analy & & 8.68 & 0.20 & $2.28 \%$ \\
\hline$C 22: 5 n 3$ & 18.2 & 0.4 & $2.42 \%$ & 17.40 & 0.70 & $4.02 \%$ & & t analy & & 18.1 & 0.5 & $2.74 \%$ \\
\hline C22:5n6 & & & & 5.41 & 0.12 & $2.19 \%$ & & t analy & & & $t$ analyz & \\
\hline$C 22: 6 n 3$ & 46.1 & 1.1 & $2.37 \%$ & 66.2 & 2.9 & $4.43 \%$ & 72.9 & 2.0 & $2.70 \%$ & 62.6 & 1.7 & $2.64 \%$ \\
\hline $\mathrm{C} 24: 0$ & & & & 22.6 & 0.9 & $4.08 \%$ & & $t$ analy & & & $t$ analyz & \\
\hline C24:1n9 & & & & 28.9 & 0.3 & $0.99 \%$ & & $t$ analy & & & $t$ analyz & \\
\hline & & & & & & & & & & & & \\
\hline List any additio & al inform & ion such & s known & elutions & r results & r additic & al fatty a & s belo & & & & \\
\hline C16:1n-7t & & & & & & & & & & & & \\
\hline C16:1n9 & & & & & & & & & & & & \\
\hline C16:2 & & & & & & & & & & & & \\
\hline C17:0 & & & & 8.19 & 0.21 & $2.62 \%$ & & & & & & \\
\hline C17:1n9 & & & & & & & & & & & & \\
\hline 9c,11t-CLA & & & & & & & & & & & & \\
\hline C18:4n-3 & & & & 0.963 & 0.047 & $4.86 \%$ & & & & & & \\
\hline C19:0 & & & & & & & & & & & & \\
\hline C $20: 3 n-3$ & & & & & & & & & & & & \\
\hline$C 20: 3 n 9$ & & & & 2.49 & 0.11 & $4.28 \%$ & & & & & & \\
\hline C2O:4n3 & & & & & & & & & & & & \\
\hline C21:0 & & & & & & & & & & & & \\
\hline C $22: 2 n-6$ & & & & & & & & & & & & \\
\hline C23:0 & & & & 10.9 & 0.2 & $1.46 \%$ & & & & & & \\
\hline C26:0 & & & & & & & & & & & & \\
\hline C26:1 & & & & & & & & & & & & \\
\hline PhA & & & & & & & & & & & & \\
\hline PrA & & & & & & & & & & & & \\
\hline & & & & & & & & & & & & \\
\hline density $(\mathrm{g} / \mathrm{mL})$ & & t analyz & & 0.967 & 0.002 & $0.18 \%$ & & tanaly & & 1.04 & 0.01 & $0.56 \%$ \\
\hline${ }^{\mathrm{a}}$ Reported as $\mu$ & & & & & & & & & & & & \\
\hline
\end{tabular}




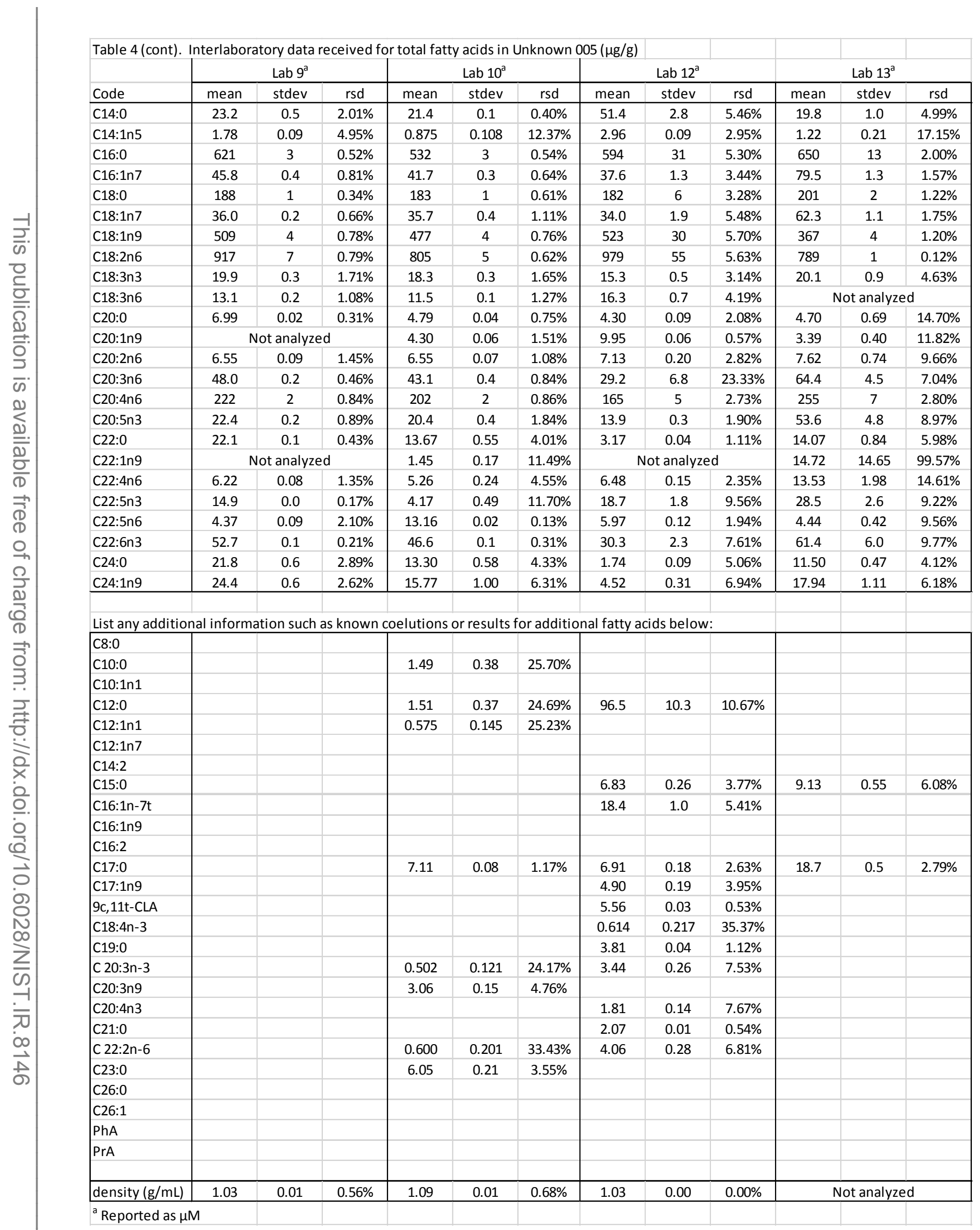




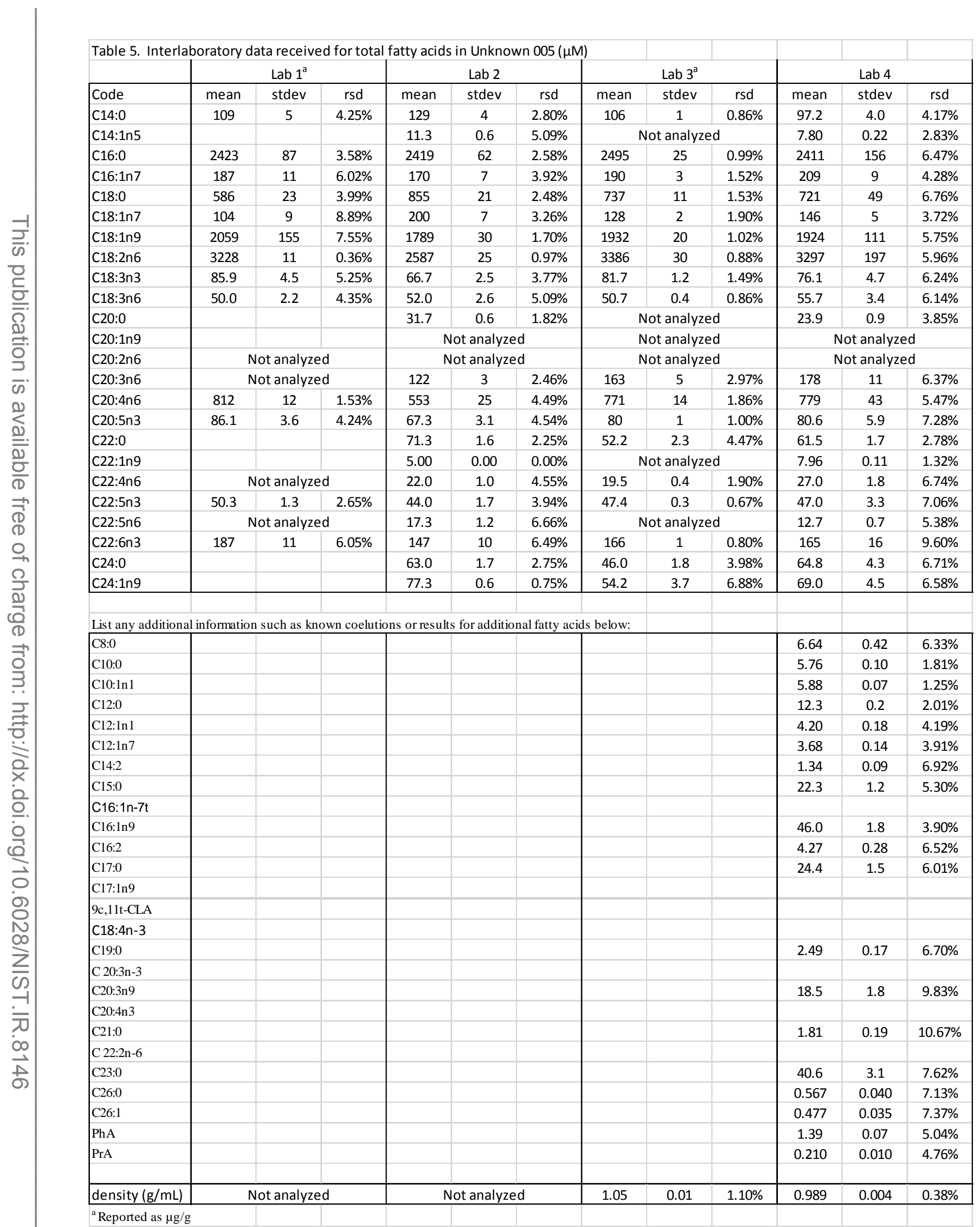




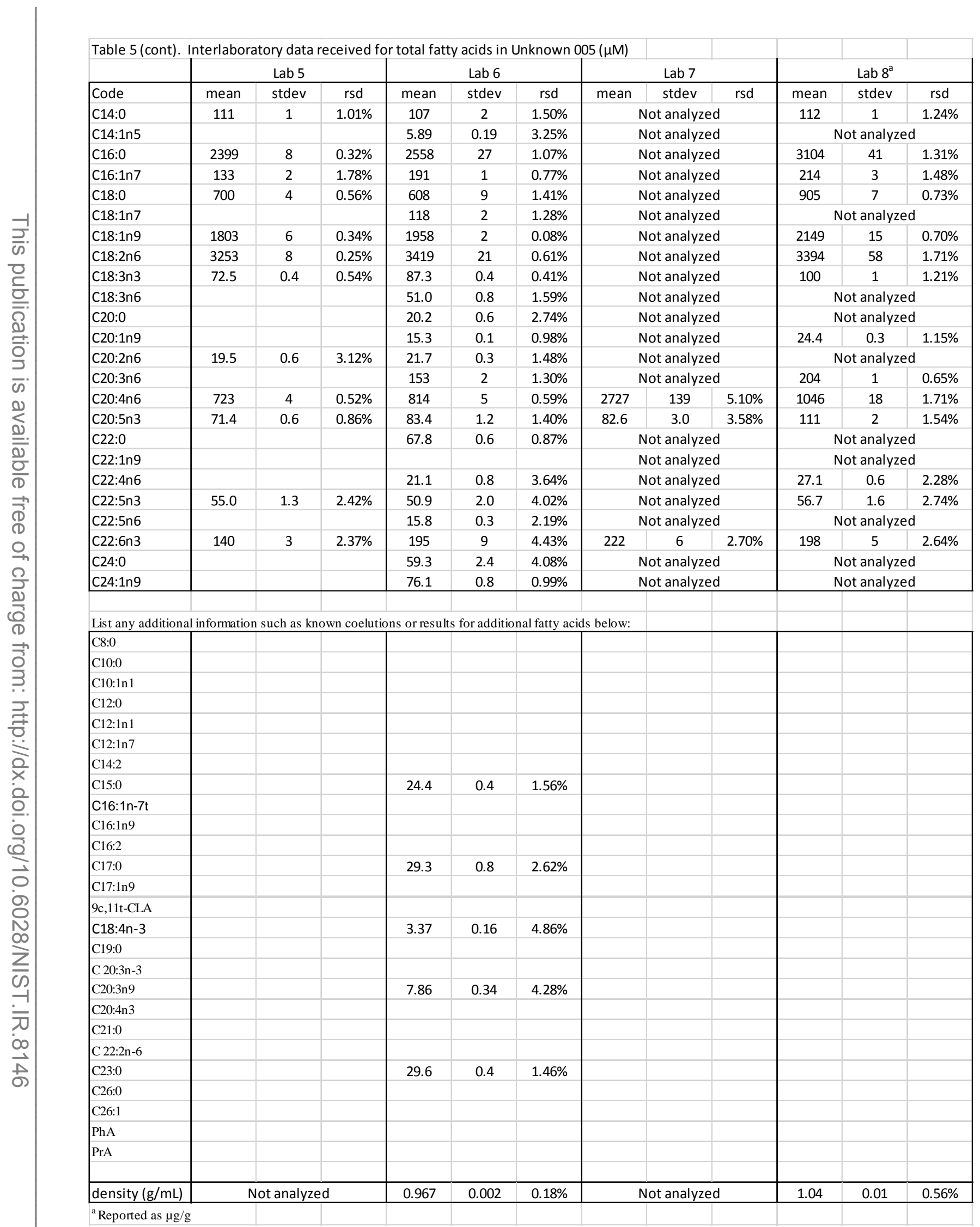




\begin{tabular}{|c|c|c|c|c|c|c|c|c|c|c|c|c|}
\hline & \multicolumn{3}{|c|}{ Lab 9} & \multicolumn{3}{|c|}{$\operatorname{Lab} 10$} & \multicolumn{3}{|c|}{ Lab 12} & \multicolumn{3}{|c|}{ Lab 13} \\
\hline C14:0 & 104 & 2 & $1.46 \%$ & 103 & 0 & $0.37 \%$ & 231 & 12 & $5.33 \%$ & 86.9 & 4.3 & $4.99 \%$ \\
\hline C14:1n5 & 8.09 & 0.36 & $4.39 \%$ & 4.22 & 0.52 & $12.37 \%$ & 13.4 & 0.4 & $2.95 \%$ & 5.39 & 0.92 & $17.15 \%$ \\
\hline C16:0 & 2487 & 12 & $0.46 \%$ & 2264 & 12 & $0.54 \%$ & 2375 & 126 & $5.30 \%$ & 2535 & 51 & $2.00 \%$ \\
\hline C18:1n7 & 131 & 2 & $1.17 \%$ & 138 & 2 & $1.11 \%$ & 123 & 7 & $5.48 \%$ & 220 & 4 & $1.75 \%$ \\
\hline C18:1n9 & 1850 & 10 & $0.54 \%$ & 1843 & 14 & $0.76 \%$ & 1896 & 108 & $5.70 \%$ & 1297 & 16 & $1.20 \%$ \\
\hline C18:2n6 & 3357 & 15 & $0.46 \%$ & 3132 & 19 & $0.62 \%$ & 3577 & 201 & $5.63 \%$ & 2813 & 3 & $0.12 \%$ \\
\hline C18:3n3 & 73.3 & 1.2 & $1.57 \%$ & 71.8 & 1.2 & $1.65 \%$ & 56.3 & 1.8 & $3.14 \%$ & 72.0 & 3.3 & $4.63 \%$ \\
\hline C18:3n6 & 48.4 & 0.9 & $1.84 \%$ & 45.0 & 0.6 & $1.27 \%$ & 59.9 & 2.5 & $4.19 \%$ & \multicolumn{3}{|c|}{ Not analyzed } \\
\hline C2O:0 & 23.0 & 0.1 & $0.25 \%$ & 16.7 & 0.1 & $0.75 \%$ & 14.1 & 0.3 & $2.08 \%$ & 15.0 & 2.2 & $14.70 \%$ \\
\hline $\mathrm{C} 20: 5 \mathrm{n} 3$ & 76.1 & 1.0 & $1.26 \%$ & 73.7 & 1.4 & $1.84 \%$ & 47.2 & 0.9 & $1.90 \%$ & 177 & 16 & $8.97 \%$ \\
\hline C22:0 & 66.5 & 0.3 & $0.40 \%$ & 43.8 & 1.8 & $4.01 \%$ & 9.54 & 0.11 & $1.11 \%$ & 41.3 & 2.5 & $5.98 \%$ \\
\hline C22:1n9 & \multicolumn{3}{|c|}{ Not analyzed } & 4.67 & 0.54 & $11.49 \%$ & \multicolumn{3}{|c|}{ Not analyzed } & 43.5 & 43.3 & $99.57 \%$ \\
\hline C22:4n6 & 19.2 & 0.4 & $1.88 \%$ & 17.3 & 0.8 & $4.55 \%$ & 20.0 & 0.5 & $2.35 \%$ & 40.7 & 5.9 & $14.61 \%$ \\
\hline C22:5n3 & 46.1 & 0.2 & $0.45 \%$ & 13.8 & 1.6 & $11.70 \%$ & 57.9 & 5.5 & $9.56 \%$ & 86.2 & 8.0 & $9.22 \%$ \\
\hline C22:5n6 & 13.6 & 0.4 & $2.59 \%$ & 43.5 & 0.1 & $0.13 \%$ & 18.5 & 0.4 & $1.94 \%$ & 13.4 & 1.3 & $9.56 \%$ \\
\hline $\mathrm{C} 22: 6 \mathrm{n} 3$ & 165 & 1 & $0.35 \%$ & 155 & 0 & $0.31 \%$ & 94.7 & 7.2 & $7.61 \%$ & 187 & 18 & $9.77 \%$ \\
\hline C24:0 & 60.7 & 2.1 & $3.44 \%$ & 39.4 & 1.7 & $4.33 \%$ & 4.84 & 0.24 & $5.06 \%$ & 31.2 & 1.3 & $4.12 \%$ \\
\hline C24:1n9 & 68.3 & 2.2 & $3.17 \%$ & 47.0 & 3.0 & $6.31 \%$ & 12.6 & 0.9 & $6.94 \%$ & 48.9 & 3.0 & $6.18 \%$ \\
\hline & & & & & & & & & & & & \\
\hline \multicolumn{13}{|c|}{ List any additional information such as known coelutions or results for additional fatty acids below: } \\
\hline \multicolumn{13}{|c|}{\begin{tabular}{|l|l|l|l|l|l|} 
C8:0 & & & & \\
\end{tabular}} \\
\hline C16:1n-7t & & & & & & & 73.9 & 4.0 & $5.41 \%$ & & & \\
\hline C16:1n9 & & & & & & & & & & & & \\
\hline C16:2 & & & & & & & & & & & & \\
\hline C17:0 & & & & 28.7 & 0.3 & $1.17 \%$ & 26.2 & 0.7 & $2.63 \%$ & 69.3 & 1.9 & $2.79 \%$ \\
\hline C17:1n9 & & & & & & & 18.7 & 0.7 & $3.95 \%$ & & & \\
\hline 9c,11t-CLA & & & & & & & 20.3 & 0.1 & $0.53 \%$ & & & \\
\hline C18:4n-3 & & & & & & & 2.28 & 0.81 & $35.37 \%$ & & & \\
\hline C19:0 & & & & & & & 13.1 & 0.1 & $1.12 \%$ & & & \\
\hline C $20: 3 n-3$ & & & & 1.79 & 0.43 & $24.17 \%$ & 11.5 & 0.9 & $7.53 \%$ & & & \\
\hline C20:3n9 & & & & 10.9 & 0.5 & $4.76 \%$ & & & & & & \\
\hline C20:4n3 & & & & & & & 6.10 & 0.47 & $7.67 \%$ & & & \\
\hline C21:0 & & & & & & & 6.48 & 0.03 & $0.54 \%$ & & & \\
\hline C $22: 2 n-6$ & & & & 1.95 & 0.65 & $33.43 \%$ & 12.4 & 0.8 & $6.81 \%$ & & & \\
\hline C23:0 & & & & 18.6 & 0.7 & $3.55 \%$ & & & & & & \\
\hline C26:0 & & & & & & & & & & & & \\
\hline C26:1 & & & & & & & & & & & & \\
\hline $\mathrm{PhA}$ & & & & & & & & & & & & \\
\hline PrA & & & & & & & & & & & & \\
\hline & & & & & & & & & & & & \\
\hline density $(\mathrm{g} / \mathrm{mL})$ & 1.03 & 0.01 & $0.56 \%$ & 1.09 & 0.01 & $0.68 \%$ & 1.03 & 0.00 & $0.00 \%$ & & $t$ analyz & \\
\hline${ }^{\mathrm{a}}$ Reported as $\mu \mathrm{g} / \mathrm{g}$ & & & & & & & & & & & & \\
\hline
\end{tabular}




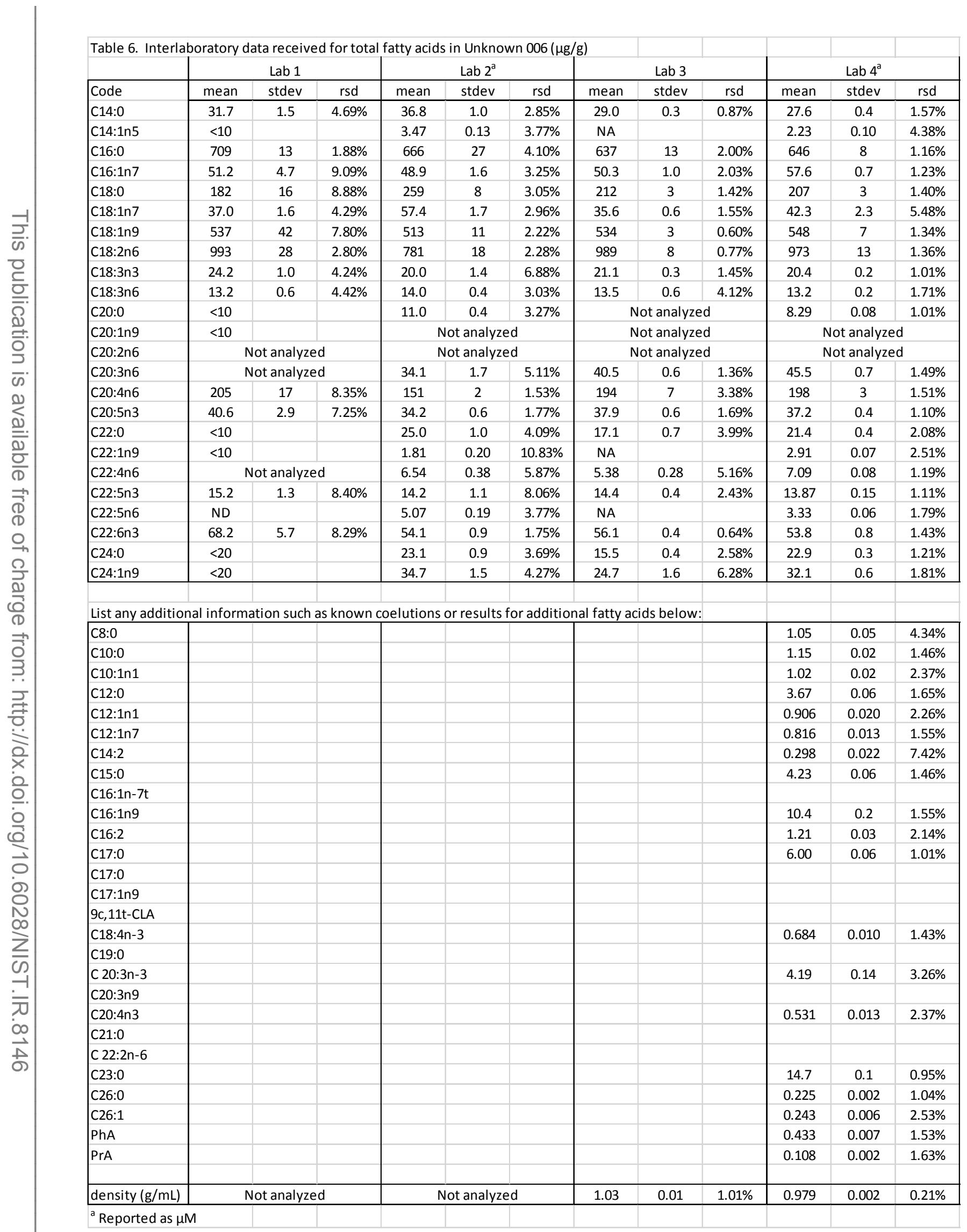




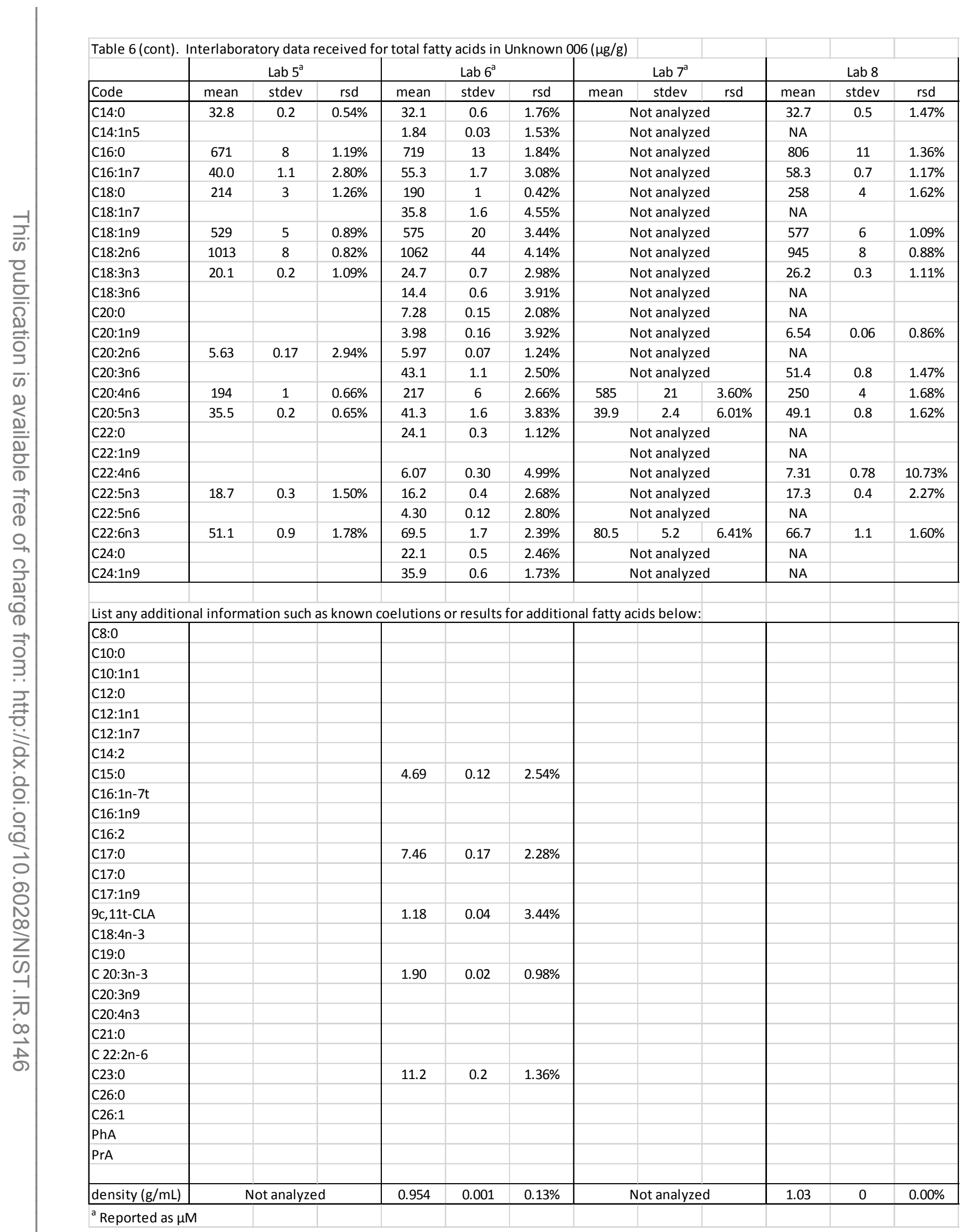




\begin{tabular}{|c|c|c|c|c|c|c|c|c|c|c|c|c|}
\hline \multicolumn{8}{|c|}{ Table 6 (cont). Interlaboratory data received for total fatty acids in Unknown $006(\mu \mathrm{g} / \mathrm{g})$} & & & & & \\
\hline & \multicolumn{3}{|c|}{$\operatorname{Lab} 9^{a}$} & \multicolumn{3}{|c|}{$\operatorname{Lab} 10^{\mathrm{a}}$} & \multicolumn{3}{|c|}{$\operatorname{Lab} 12^{\mathrm{a}}$} & \multicolumn{3}{|c|}{ Lab $13^{\mathrm{a}}$} \\
\hline C14:0 & 29.2 & 0.1 & $0.40 \%$ & 26.7 & 0.2 & $0.65 \%$ & 58.7 & 4.5 & $7.69 \%$ & 24.4 & 1.1 & $4.57 \%$ \\
\hline C14:1n5 & 2.38 & 0.05 & $1.90 \%$ & 1.35 & 0.14 & $10.13 \%$ & 3.65 & 0.62 & $17.02 \%$ & 1.71 & 0.21 & $12.52 \%$ \\
\hline C16:0 & 661 & 4 & $0.57 \%$ & 561 & 2 & $0.29 \%$ & 652 & 48 & $7.37 \%$ & 665 & 28 & $4.17 \%$ \\
\hline C18:1n7 & 37.1 & 0.1 & $0.14 \%$ & 35.6 & 0.1 & $0.30 \%$ & 38.4 & 2.1 & $5.43 \%$ & 63.8 & 0.8 & $1.20 \%$ \\
\hline C18:1n9 & 518 & 3 & $0.49 \%$ & 485 & 1 & $0.20 \%$ & 548 & 37 & $6.80 \%$ & 356 & 7 & $1.87 \%$ \\
\hline C18:2n6 & 995 & 5 & $0.49 \%$ & 872 & 1 & $0.08 \%$ & 1113 & 87 & $7.83 \%$ & 821 & 8 & $1.03 \%$ \\
\hline C18:3n3 & 20.2 & 0.3 & $1.65 \%$ & 18.1 & 0.1 & $0.59 \%$ & 16.2 & 1.3 & $8.26 \%$ & 16.7 & 0.6 & $3.56 \%$ \\
\hline C18:3n6 & 13.2 & 0.2 & $1.58 \%$ & 11.3 & 0.1 & $0.72 \%$ & 17.8 & 1.1 & $6.05 \%$ & \multicolumn{3}{|c|}{ Not analyzed } \\
\hline C2O:0 & 7.84 & 0.10 & $1.30 \%$ & 5.23 & 0.35 & $6.65 \%$ & 4.70 & 0.48 & $10.11 \%$ & 4.83 & 1.22 & $25.24 \%$ \\
\hline C20:5n3 & 36.3 & 0.2 & $0.43 \%$ & 33.2 & 0.1 & $0.32 \%$ & 23.7 & 2.6 & $11.10 \%$ & 65.3 & 1.0 & $1.58 \%$ \\
\hline C22:0 & 22.1 & 0.3 & $1.21 \%$ & 13.6 & 0.6 & $4.11 \%$ & 3.69 & 0.37 & $10.03 \%$ & 12.9 & 2.2 & $16.85 \%$ \\
\hline C22:1n9 & \multicolumn{3}{|c|}{ Not analyzed } & 1.71 & 0.16 & $9.54 \%$ & \multicolumn{3}{|c|}{ Not analyzed } & 11.2 & 10.2 & $90.29 \%$ \\
\hline C22:4n6 & 5.19 & 0.09 & $1.72 \%$ & 4.50 & 0.20 & $4.47 \%$ & 6.00 & 1.11 & $18.51 \%$ & 11.6 & 0.8 & $6.74 \%$ \\
\hline$C 22: 5 n 3$ & 14.04 & 0.17 & $1.19 \%$ & 3.69 & 0.31 & $8.32 \%$ & 19.9 & 1.9 & $9.55 \%$ & 24.5 & 2.1 & $8.40 \%$ \\
\hline$C 22: 5 n 6$ & 3.69 & 0.08 & $2.17 \%$ & 12.6 & 0.3 & $2.42 \%$ & 6.23 & 0.49 & $7.92 \%$ & 3.42 & 0.16 & $4.57 \%$ \\
\hline $\mathrm{C} 22: 6 \mathrm{n} 3$ & 57.1 & 0.4 & $0.61 \%$ & 50.0 & 0.1 & $0.18 \%$ & 35.6 & 7.3 & $20.42 \%$ & 59.2 & 2.8 & $4.64 \%$ \\
\hline C24:0 & 20.8 & 0.3 & $1.51 \%$ & 12.2 & 0.6 & $5.32 \%$ & 2.15 & 0.19 & $8.63 \%$ & 10.5 & 2.3 & $22.35 \%$ \\
\hline C24:1n9 & 30.9 & 0.6 & $1.85 \%$ & 19.1 & 0.9 & $4.97 \%$ & 5.26 & 0.97 & $18.47 \%$ & 21.1 & 5.9 & $28.10 \%$ \\
\hline \multicolumn{12}{|c|}{ List any additional information such as known coelutions or results for additional fatty acids below: } & \\
\hline \multicolumn{13}{|c|}{\begin{tabular}{|l|l|l|l|l|l|l|}
$\mathrm{C} 8: 0$ & & & & & & \\
\end{tabular}} \\
\hline C16:1n-7t & & & & & & & 18.6 & 0.9 & $5.10 \%$ & & & \\
\hline C16:1n9 & & & & & & & & & & & & \\
\hline C16:2 & & & & & & & & & & & & \\
\hline C17:0 & & & & 6.58 & 0.05 & $0.79 \%$ & 6.93 & 0.52 & $7.46 \%$ & 15.7 & 1.1 & $6.74 \%$ \\
\hline C17:0 & & & & & & & 5.27 & 0.44 & $8.29 \%$ & & & \\
\hline C17:1n9 & & & & & & & & & & & & \\
\hline 9c,11t-CLA & & & & & & & 1.42 & 0.20 & $13.98 \%$ & & & \\
\hline C18:4n-3 & & & & & & & 4.11 & 0.47 & $11.38 \%$ & & & \\
\hline C19:0 & & & & 0.550 & 0.066 & $12.03 \%$ & 3.61 & 0.47 & $12.94 \%$ & & & \\
\hline C $20: 3 n-3$ & & & & 2.48 & 0.10 & $4.04 \%$ & & & & & & \\
\hline C20:3n9 & & & & & & & 1.46 & 0.33 & $22.50 \%$ & & & \\
\hline C20:4n3 & & & & & & & 2.10 & 0.22 & $10.26 \%$ & & & \\
\hline C21:0 & & & & 0.464 & 0.194 & $41.93 \%$ & 4.60 & 0.52 & $11.23 \%$ & & & \\
\hline C $22: 2 n-6$ & & & & 0.478 & 0.200 & $41.93 \%$ & 4.74 & 0.53 & $11.23 \%$ & & & \\
\hline C23:0 & & & & 5.70 & 0.39 & $6.89 \%$ & & & & & & \\
\hline $\mathrm{C} 26: 0$ & & & & & & & & & & & & \\
\hline C26:1 & & & & & & & & & & & & \\
\hline $\mathrm{PhA}$ & & & & & & & & & & & & \\
\hline PrA & & & & & & & & & & & & \\
\hline & & & & & & & & & & & & \\
\hline density $(\mathrm{g} / \mathrm{mL})$ & 1.02 & 0.01 & $0.57 \%$ & 1.09 & 0.00 & $0.40 \%$ & 1.03 & 0.00 & $0.00 \%$ & & tanaly & \\
\hline${ }^{\mathrm{a}}$ Reported as $\mu$ & & & & & & & & & & & & \\
\hline
\end{tabular}




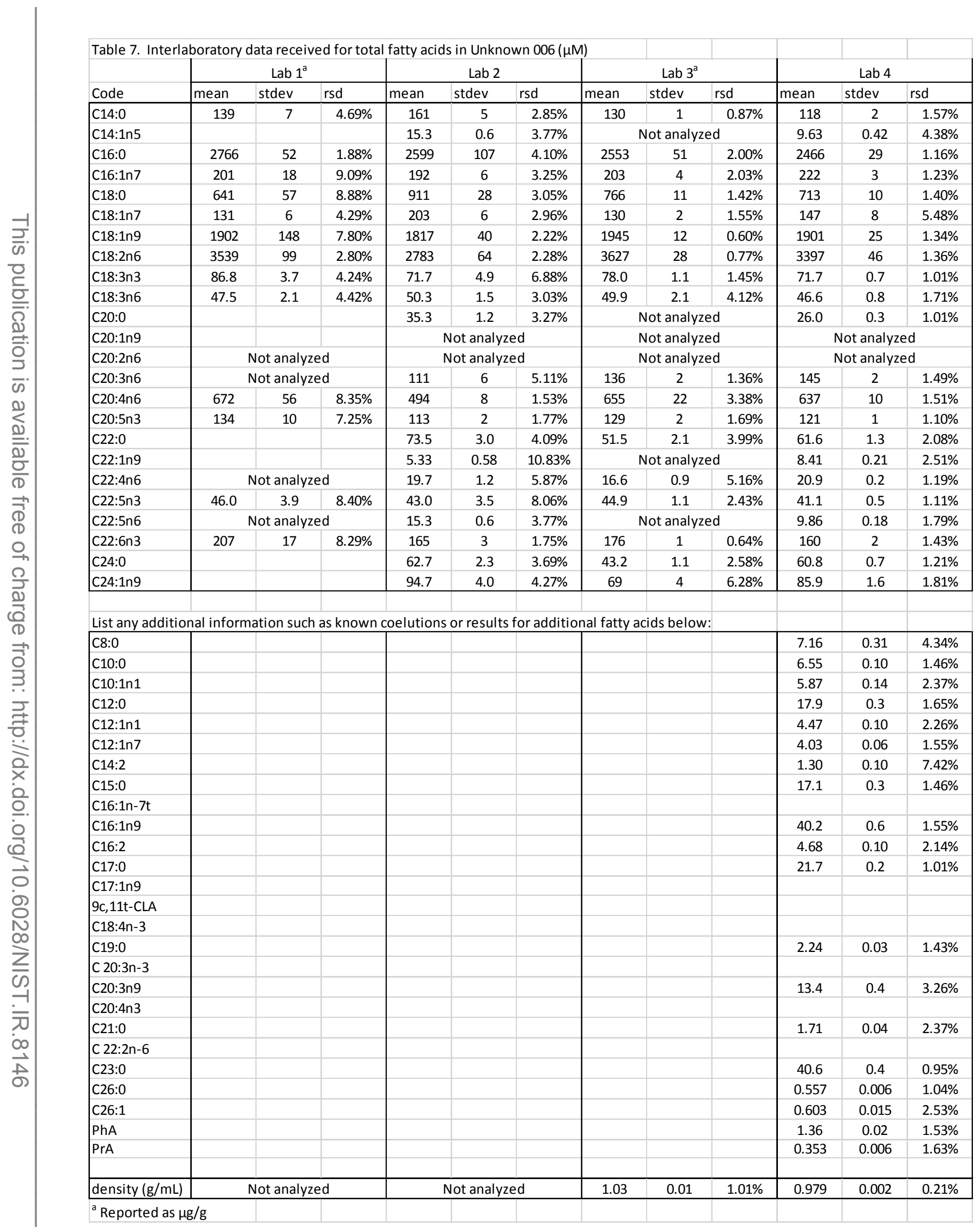




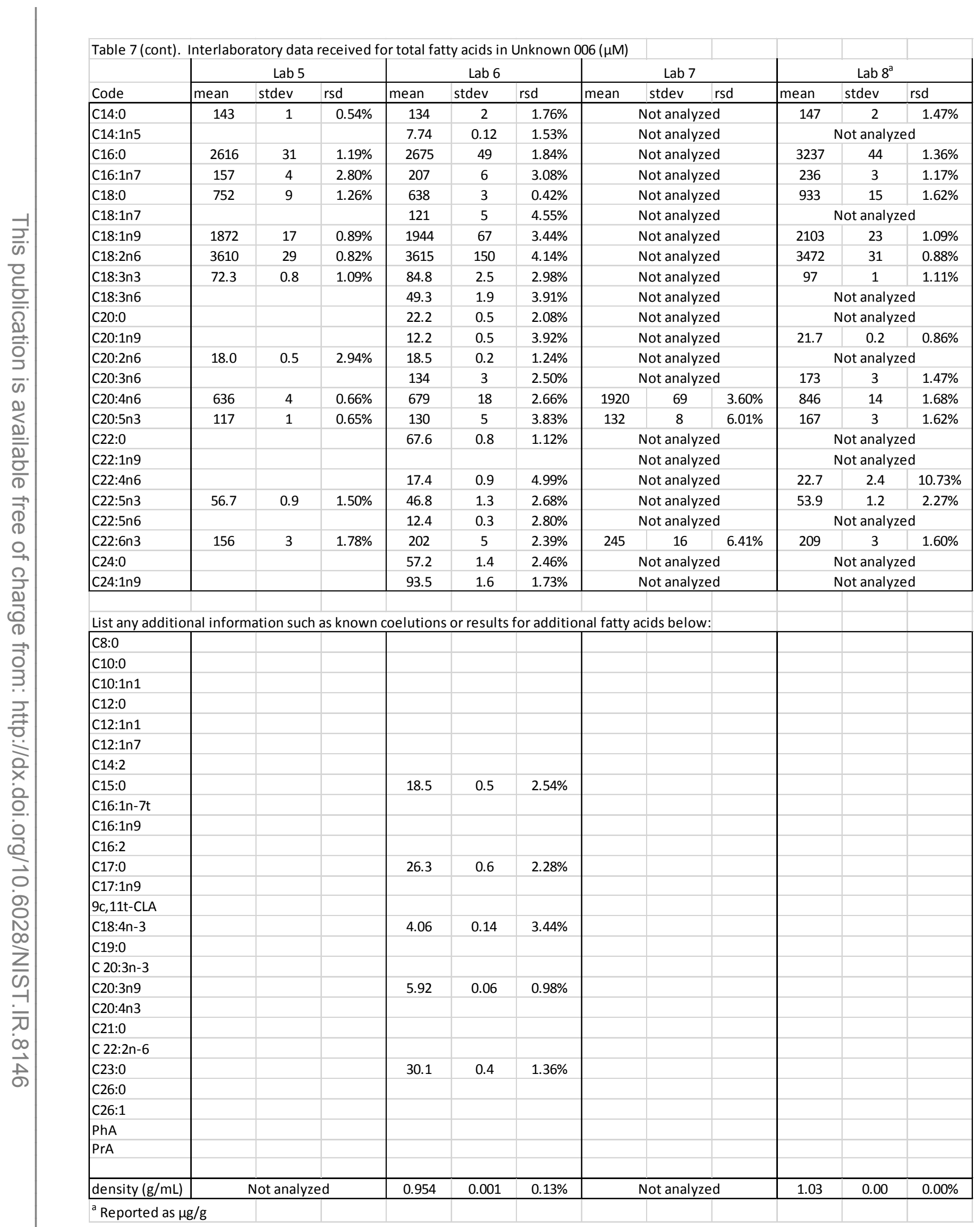




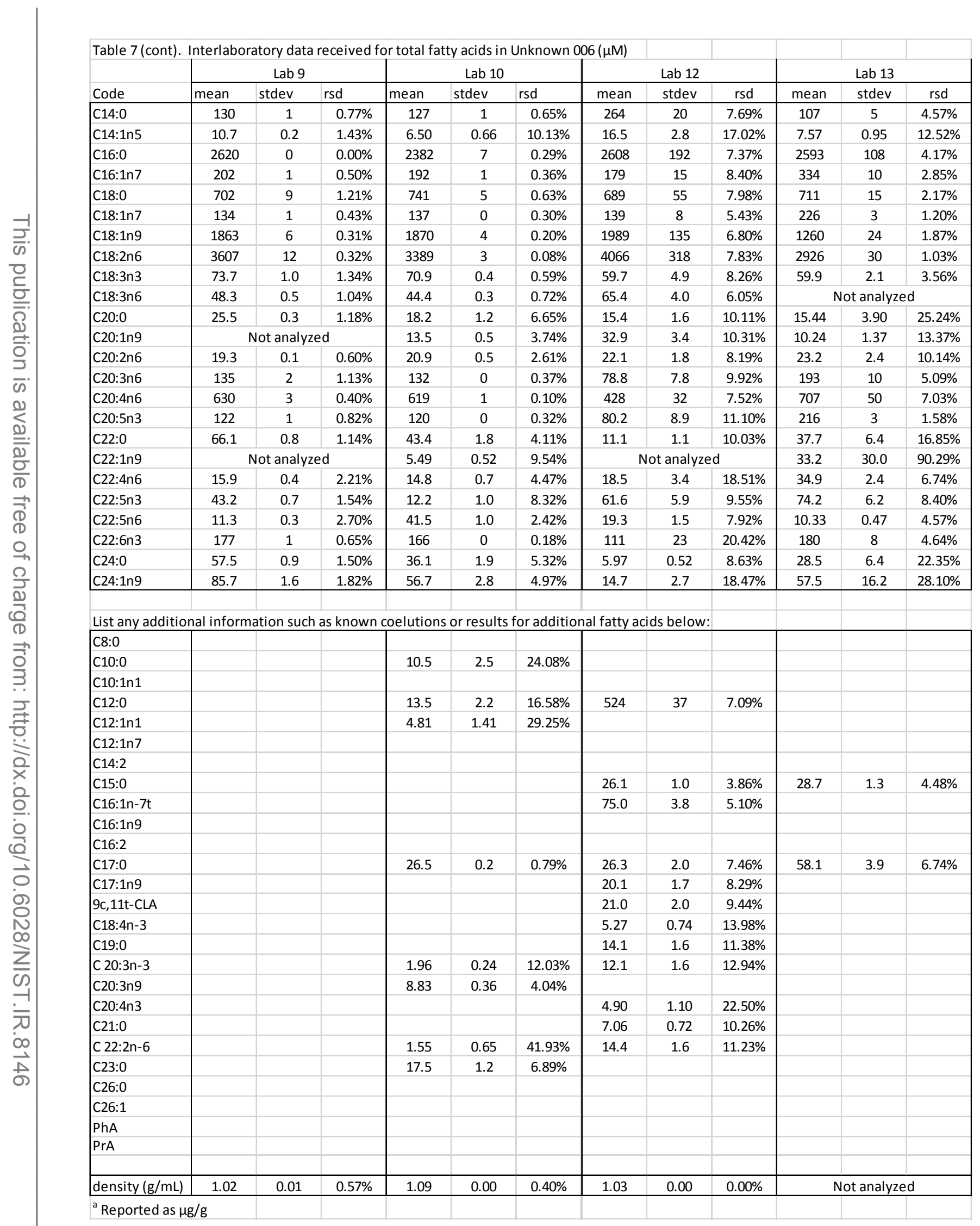




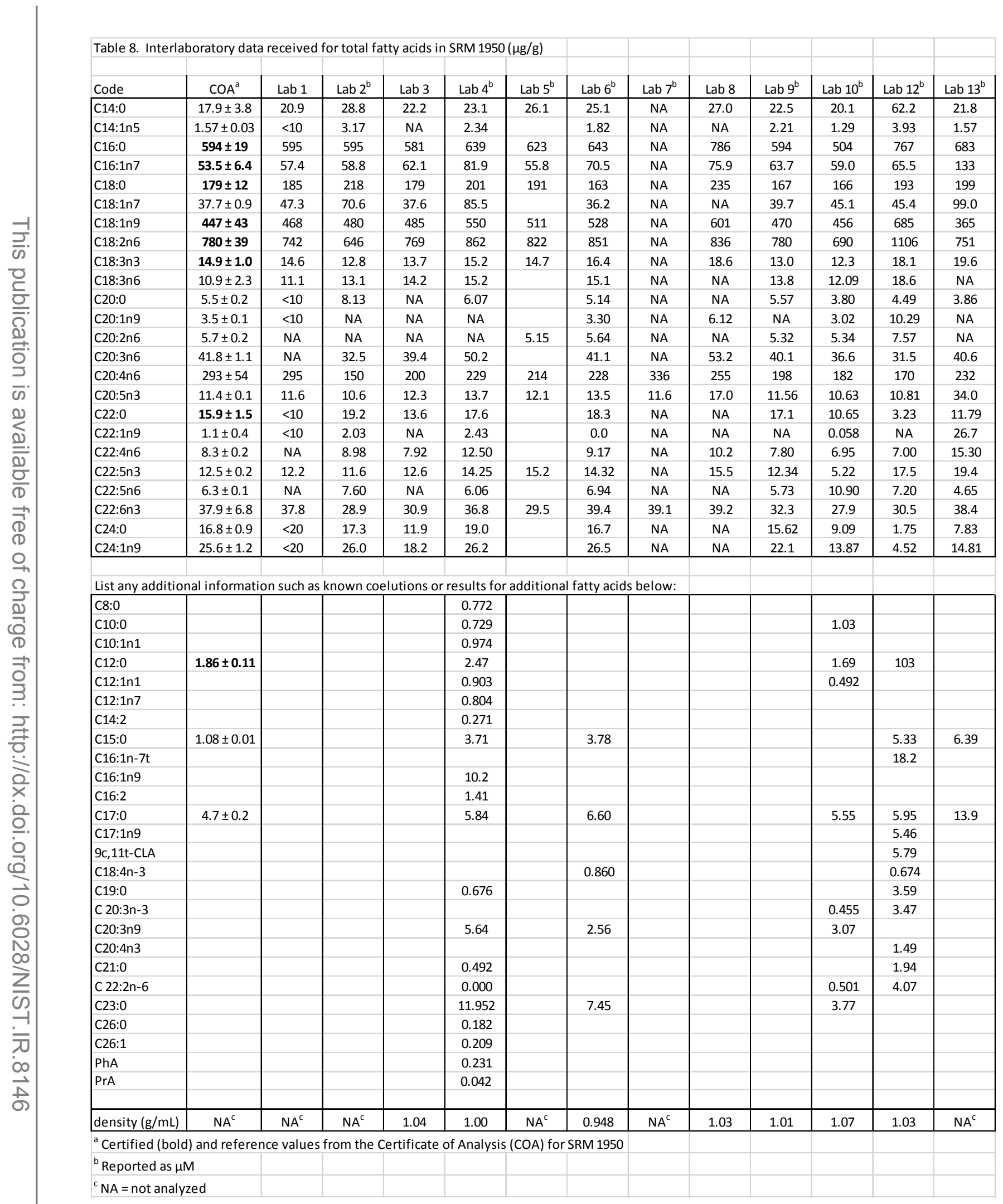




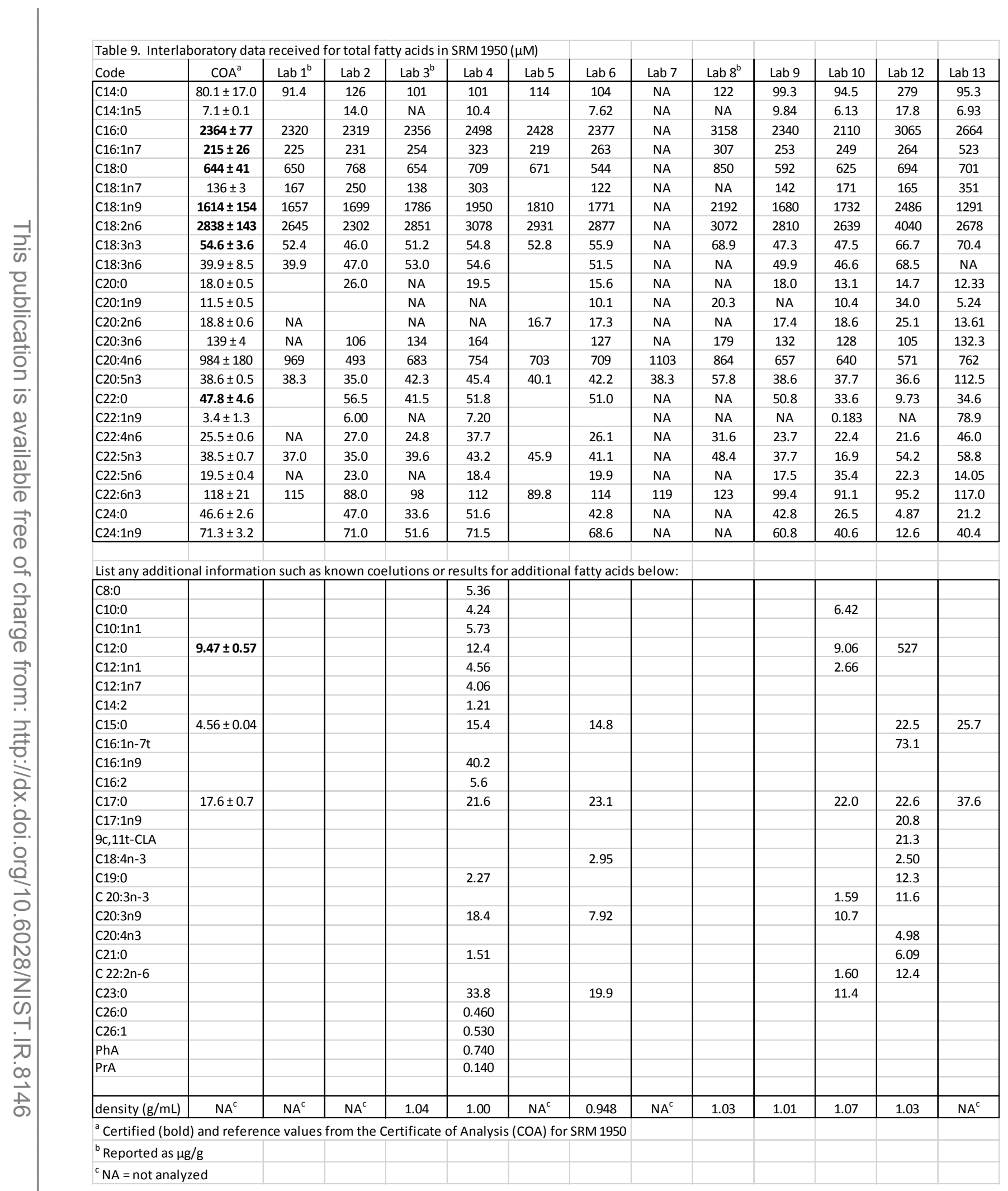


Table 10. Summary statistics for total fatty acids $(\mu \mathrm{M})$

Unknown 004

\begin{tabular}{|l|c|c|c|c|c|c|c|c|}
\hline \multirow{2}{*}{ Code } & median & unc & rel unc & $\mathrm{n}^{\mathrm{a}}$ & \multicolumn{2}{|c|}{ lowest value } & \multicolumn{2}{|c|}{ highest value } \\
\hline C14:0 & 161 & 33 & $20.7 \%$ & 11 & 106 & Lab 13 & 279 & Lab 12 \\
\hline C14:1n5 & 13.9 & 4.7 & $33.4 \%$ & 7 & 7.80 & Lab 13 & 21.0 & Lab 2 \\
\hline C16:0 & 3048 & 291 & $9.6 \%$ & 11 & 2715 & Lab 10 & 4202 & Lab 8 \\
\hline C16:1n7 & 297 & 39 & $13.1 \%$ & 11 & 246 & Lab 5 & 413 & Lab 13 \\
\hline C18:0 & 716 & 89 & $12.4 \%$ & 11 & 620 & Lab 6 & 998 & Lab 8 \\
\hline C18:1n7 & 177 & 45 & $25.4 \%$ & 9 & 151 & Lab 6 & 294 & Lab 2 \\
\hline C18:1n9 & 2306 & 292 & $12.7 \%$ & 11 & 1440 & Lab 13 & 3128 & Lab 8 \\
\hline C18:2n6 & 3531 & 296 & $8.4 \%$ & 11 & 2766 & Lab 13 & 4040 & Lab 12 \\
\hline C18:3n3 & 85.6 & 14.6 & $17.1 \%$ & 11 & 57.2 & Lab 13 & 132 & Lab 8 \\
\hline C18:3n6 & 55.7 & 4.9 & $8.8 \%$ & 8 & 49.1 & Lab 10 & 68.5 & Lab 12 \\
\hline C20:0 & 20.3 & 6.3 & $31.2 \%$ & 7 & 12.9 & Lab 13 & 32.7 & Lab 2 \\
\hline C20:1n9 & 17.3 & 11.4 & $65.9 \%$ & 5 & 10.2 & Lab 13 & 34.0 & Lab 12 \\
\hline C20:2n6 & 24.3 & 2.6 & $10.6 \%$ & 6 & 19.1 & Lab 5 & 25.1 & Lab 12 \\
\hline C20:3n6 & 164 & 29 & $17.8 \%$ & 9 & 105 & Lab 12 & 229 & Lab 8 \\
\hline C20:4n6 & 722 & 447 & $61.8 \%$ & 12 & 567 & Lab 2 & 2818 & Lab 7 \\
\hline C20:5n3 & 59.8 & 15.4 & $25.8 \%$ & 12 & 36.6 & Lab 12 & 110 & Lab 13 \\
\hline C22:0 & 53.7 & 18.2 & $34.0 \%$ & 8 & 9.73 & Lab 12 & 70.6 & Lab 2 \\
\hline C22:1n9 & 10.3 & 2.8 & $26.9 \%$ & 4 & 9.26 & Lab 10 & 14.2 & Lab 13 \\
\hline C22:4n6 & 25.8 & 4.6 & $17.8 \%$ & 9 & 21.1 & Lab 10 & 35.1 & Lab 13 \\
\hline C22:5n3 & 48.7 & 7.9 & $16.3 \%$ & 11 & 19.0 & Lab 10 & 57.5 & Lab 8 \\
\hline C22:5n6 & 21.3 & 8.8 & $41.3 \%$ & 7 & 12.3 & Lab 13 & 41.6 & Lab 10 \\
\hline C22:6n3 & 139 & 27 & $19.5 \%$ & 12 & 57.9 & Lab 8 & 214 & Lab 7 \\
\hline C24:0 & 46.0 & 16.7 & $36.4 \%$ & 8 & 4.87 & Lab 12 & 58.0 & Lab 2 \\
\hline C24:1n9 & 64.3 & 21.7 & $33.7 \%$ & 8 & 12.6 & Lab 12 & 86.0 & Lab 2 \\
\hline
\end{tabular}

List any additional information such as known coelutions or results for additional fatty acids below:

\begin{tabular}{|l|c|c|c|c|c|c|c|c|}
\hline C15:0 & 26.5 & 5.6 & $21.1 \%$ & 4 & 22.1 & Lab 4 & 31.6 & Lab 13 \\
\hline C17:0 & 28.6 & 15.8 & $55.2 \%$ & 5 & 24.9 & Lab 4 & 59.1 & Lab 13 \\
\hline
\end{tabular}

\begin{tabular}{|l|l|l|l|l|l|l|l|l|}
\hline density $(\mathrm{g} / \mathrm{mL})$ & 1.03 & 0.03 & $2.9 \%$ & 7 & 0.963 & Lab 6 & 1.06 & Lab 3 \\
\hline
\end{tabular}

${ }^{a} \mathrm{n}$ is number of laboratories reporting a value. 
Table 10 (cont). Summary statistics for total fatty acids $(\mu \mathrm{M})$

\section{Unknown 005}

\begin{tabular}{|l|c|c|c|c|c|c|c|c|}
\cline { 2 - 10 } Code & median & unc & rel unc & $\mathrm{n}^{\mathrm{a}}$ & \multicolumn{2}{|c|}{ lowest value } & \multicolumn{2}{|c|}{ highest value } \\
\hline C14:0 & 107 & 29 & $27.3 \%$ & 11 & 86.9 & Lab 13 & 231 & Lab 12 \\
\hline C14:1n5 & 7.80 & 3.14 & $40.2 \%$ & 7 & 4.22 & Lab 10 & 13.4 & Lab 12 \\
\hline C16:0 & 2423 & 164 & $6.8 \%$ & 11 & 2264 & Lab 10 & 3104 & Lab 8 \\
\hline C16:1n7 & 187 & 35 & $18.6 \%$ & 11 & 133 & Lab 5 & 312 & Lab 13 \\
\hline C18:0 & 701 & 71 & $10.2 \%$ & 11 & 586 & Lab 1 & 905 & Lab 8 \\
\hline C18:1n7 & 131 & 32 & $24.8 \%$ & 9 & 104 & Lab 1 & 220 & Lab 13 \\
\hline C18:1n9 & 1896 & 163 & $8.6 \%$ & 11 & 1297 & Lab 13 & 2149 & Lab 8 \\
\hline C18:2n6 & 3297 & 216 & $6.6 \%$ & 11 & 2587 & Lab 2 & 3577 & Lab 12 \\
\hline C18:3n3 & 73.3 & 8.8 & $12.0 \%$ & 11 & 56.2 & Lab 12 & 100 & Lab 8 \\
\hline C18:3n6 & 50.9 & 4.0 & $7.9 \%$ & 8 & 45.0 & Lab 10 & 60.0 & Lab 12 \\
\hline C20:0 & 20.2 & 5.8 & $28.8 \%$ & 7 & 14.1 & Lab 12 & 31.7 & Lab 2 \\
\hline C20:1n9 & 15.3 & 9.9 & $64.7 \%$ & 5 & 10.9 & Lab 13 & 32.9 & Lab 12 \\
\hline C20:2n6 & 22.5 & 1.8 & $7.9 \%$ & 6 & 19.5 & Lab 5 & 24.4 & Lab 13 \\
\hline C20:3n6 & 161 & 30 & $18.6 \%$ & 9 & 97.5 & Lab 12 & 210 & Lab 13 \\
\hline C20:4n6 & 775 & 420 & $54.2 \%$ & 12 & 553 & Lab 2 & 2727 & Lab 7 \\
\hline C20:5n3 & 80.4 & 23.1 & $28.8 \%$ & 12 & 47.2 & Lab 12 & 177 & Lab 13 \\
\hline C22:0 & 56.9 & 18.0 & $31.7 \%$ & 8 & 9.54 & Lab 12 & 71.3 & Lab 2 \\
\hline C22:1n9 & 6.48 & 23.6 & $363.8 \%$ & 4 & 4.67 & Lab 10 & 43.5 & Lab 13 \\
\hline C22:4n6 & 21.1 & 6.0 & $28.4 \%$ & 9 & 17.3 & Lab 10 & 40.7 & Lab 13 \\
\hline C22:5n3 & 50.3 & 12.7 & $25.2 \%$ & 11 & 13.8 & Lab 10 & 86.2 & Lab 13 \\
\hline C22:5n6 & 15.8 & 10.3 & $65.1 \%$ & 7 & 12.7 & Lab 4 & 43.5 & Lab 10 \\
\hline C22:6n3 & 166 & 24 & $14.4 \%$ & 12 & 94.7 & Lab 12 & 222 & Lab 7 \\
\hline C24:0 & 52.7 & 18.3 & $34.7 \%$ & 8 & 4.84 & Lab 12 & 64.8 & Lab 4 \\
\hline C24:1n9 & 61.3 & 18.9 & $30.8 \%$ & 8 & 12.6 & Lab 12 & 77.3 & Lab 2 \\
\hline
\end{tabular}

List any additional information such as known coelutions or results for additional fatty acids below:

\begin{tabular}{|l|c|c|c|c|c|c|c|c|}
\hline C15:0 & 26.6 & 8.5 & $32.1 \%$ & 4 & 22.3 & Lab 4 & 37.7 & Lab 13 \\
\hline C17:0 & 28.7 & 21.2 & $73.9 \%$ & 5 & 24.4 & Lab 4 & 69.3 & Lab 13 \\
\hline & & & & & & & \\
\hline density $(\mathrm{g} / \mathrm{mL})$ & 1.03 & 0.04 & $3.9 \%$ & 7 & 0.967 & Lab 6 & 1.09 & Lab 10 \\
\hline
\end{tabular}

${ }^{a} \mathrm{n}$ is number of laboratories reporting a value. 
Table 10 (cont). Summary statistics for total fatty acids $(\mu M)$

Unknown 006

\begin{tabular}{|l|c|c|c|c|c|c|c|c|}
\hline & median & unc & rel unc & $\mathrm{n}^{\text {a }}$ & \multicolumn{2}{|c|}{ lowest value } & \multicolumn{2}{|c|}{ highest value } \\
\hline C14:0 & 134 & 10 & $7.3 \%$ & 11 & 107 & Lab 13 & 264 & Lab 12 \\
\hline C14:1n5 & 9.63 & 3.8 & $40.0 \%$ & 7 & 6.50 & Lab 10 & 16.5 & Lab 12 \\
\hline C16:0 & 2608 & 11 & $0.4 \%$ & 11 & 2382 & Lab 10 & 3237 & Lab 8 \\
\hline C16:1n7 & 202 & 9 & $4.5 \%$ & 11 & 157 & Lab 5 & 334 & Lab 13 \\
\hline C18:0 & 713 & 10 & $1.3 \%$ & 11 & 638 & Lab 6 & 933 & Lab 8 \\
\hline C18:1n7 & 137 & 8 & $6.1 \%$ & 9 & 121 & Lab 6 & 226 & Lab 13 \\
\hline C18:1n9 & 1901 & 13 & $0.7 \%$ & 11 & 1260 & Lab 13 & 2103 & Lab 8 \\
\hline C18:2n6 & 3539 & 17 & $0.5 \%$ & 11 & 2783 & Lab 2 & 4066 & Lab 12 \\
\hline C18:3n3 & 72.3 & 3.3 & $4.6 \%$ & 11 & 59.7 & Lab 12 & 97.0 & Lab 8 \\
\hline C18:3n6 & 48.8 & 2.4 & $5.0 \%$ & 8 & 44.4 & Lab 10 & 65.4 & Lab 12 \\
\hline C20:0 & 22.2 & 4.2 & $18.7 \%$ & 7 & 15.4 & Lab 12 \& 13 & 35.3 & Lab 2 \\
\hline C20:1n9 & 13.5 & 6.7 & $49.5 \%$ & 5 & 10.2 & Lab 13 & 32.9 & Lab 12 \\
\hline C20:2n6 & 20.1 & 1.2 & $6.2 \%$ & 6 & 12.0 & Lab 5 & 23.2 & Lab 13 \\
\hline C20:3n6 & 135 & 8 & $5.7 \%$ & 9 & 78.8 & Lab 12 & 193 & Lab 13 \\
\hline C20:4n6 & 646 & 43 & $6.7 \%$ & 12 & 428 & Lab 12 & 1920 & Lab 7 \\
\hline C20:5n3 & 125 & 8 & $6.2 \%$ & 12 & 80.2 & Lab 12 & 216 & Lab 13 \\
\hline C22:0 & 56.5 & 7.8 & $13.8 \%$ & 8 & 11.1 & Lab 12 & 73.5 & Lab 2 \\
\hline C22:1n9 & 6.95 & 14.6 & $209.9 \%$ & 4 & 5.33 & Lab 2 & 33.2 & Lab 13 \\
\hline C22:4n6 & 18.5 & 4.0 & $21.4 \%$ & 9 & 14.8 & Lab 10 & 34.9 & Lab 13 \\
\hline C22:5n3 & 46.0 & 11.0 & $24.0 \%$ & 11 & 12.2 & Lab 10 & 74.2 & Lab 13 \\
\hline C22:5n6 & 12.4 & 8.9 & $72.1 \%$ & 7 & 9.86 & Lab 4 & 41.5 & Lab 10 \\
\hline C22:6n3 & 176 & 7 & $3.8 \%$ & 12 & 111 & Lab 12 & 245 & Lab 7 \\
\hline C24:0 & 50.2 & 8.2 & $16.4 \%$ & 8 & 5.97 & Lab 12 & 62.7 & Lab 2 \\
\hline C24:1n9 & 77.5 & 8.9 & $11.5 \%$ & 8 & 14.7 & Lab 12 & 94.7 & Lab 2 \\
\hline & & & & & & & & \\
\hline
\end{tabular}

List any additional information such as known coelutions or results for additional fatty acids below:

\begin{tabular}{|l|c|c|c|c|c|c|c|c|}
\hline C15:0 & 22.3 & 3.4 & $15.5 \%$ & 4 & 17.1 & Lab 4 & 28.7 & Lab 13 \\
\hline C17:0 & 26.3 & 8.0 & $30.3 \%$ & 5 & 21.7 & Lab 4 & 58.1 & Lab 13 \\
\hline & & & & & & & & \\
\hline density $(\mathrm{g} / \mathrm{mL})$ & 1.03 & 0.11 & $10.7 \%$ & 7 & 0.954 & Lab 6 & 1.09 & Lab 10 \\
\hline
\end{tabular}

${ }^{a} \mathrm{n}$ is number of laboratories reporting a value. 
Table 10 (cont). Summary statistics for total fatty acids ( $\mu \mathrm{M})$

SRM 1950

\begin{tabular}{|l|c|c|c|c|c|c|c|c|}
\cline { 2 - 10 } Code & median & unc & rel unc & $\mathrm{n}^{\mathrm{a}}$ & \multicolumn{2}{|c|}{ lowest value } & \multicolumn{2}{|c|}{ highest value } \\
\hline C14:0 & 101 & 41 & $39.9 \%$ & 11 & 91.4 & Lab 1 & 279 & Lab 12 \\
\hline C14:1n5 & 9.84 & 4.0 & $40.4 \%$ & 7 & 6.13 & Lab 10 & 17.8 & Lab 12 \\
\hline C16:0 & 2377 & 245 & $10.3 \%$ & 11 & 2110 & Lab 10 & 3158 & Lab 8 \\
\hline C16:1n7 & 254 & 65 & $25.4 \%$ & 11 & 219 & Lab 5 & 523 & Lab 13 \\
\hline C18:0 & 671 & 63 & $9.3 \%$ & 11 & 544 & Lab 6 & 850 & Lab 8 \\
\hline C18:1n7 & 167 & 67 & $40.2 \%$ & 9 & 122 & Lab 6 & 351 & Lab 13 \\
\hline C18:1n9 & 1771 & 232 & $13.1 \%$ & 11 & 1291 & Lab 13 & 2486 & Lab 12 \\
\hline C18:2n6 & 2851 & 330 & $11.6 \%$ & 11 & 2302 & Lab 2 & 4040 & Lab 12 \\
\hline C18:3n3 & 52.8 & 6.7 & $12.7 \%$ & 11 & 46.0 & Lab 2 & 70.4 & Lab 13 \\
\hline C18:3n6 & 50.7 & 7.3 & $14.5 \%$ & 8 & 46.4 & Lab 10 & 68.5 & Lab 12 \\
\hline C20:0 & 15.6 & 4.4 & $28.5 \%$ & 7 & 12.3 & Lab 13 & 26.0 & Lab 2 \\
\hline C20:1n9 & 10.4 & 12.8 & $122.6 \%$ & 5 & 5.24 & Lab 13 & 34.0 & Lab 12 \\
\hline C20:2n6 & 17.4 & 3.9 & $22.5 \%$ & 6 & 13.6 & Lab 13 & 25.1 & Lab 12 \\
\hline C20:3n6 & 132 & 20 & $15.2 \%$ & 9 & 105 & Lab 12 & 179 & Lab 8 \\
\hline C20:4n6 & 706 & 122 & $17.2 \%$ & 12 & 493 & Lab 2 & 1103 & Lab 7 \\
\hline C20:5n3 & 39.4 & 15.5 & $39.3 \%$ & 12 & 35.0 & Lab 2 & 113 & Lab 13 \\
\hline C22:0 & 46.2 & 13.5 & $29.2 \%$ & 8 & 9.73 & Lab 12 & 56.5 & Lab 2 \\
\hline C22:1n9 & 6.60 & 46.7 & $707.0 \%$ & 4 & 0.182 & Lab 10 & 78.9 & Lab 13 \\
\hline C22:4n6 & 26.1 & 6.8 & $25.9 \%$ & 9 & 21.9 & Lab 12 & 46.0 & Lab 13 \\
\hline C22:5n3 & 41.1 & 8.3 & $20.2 \%$ & 11 & 16.9 & Lab 10 & 58.8 & Lab 13 \\
\hline C22:5n6 & 19.9 & 6.4 & $32.4 \%$ & 7 & 14.1 & Lab 13 & 35.4 & Lab 10 \\
\hline C22:6n3 & 106 & 9 & $8.7 \%$ & 12 & 88.0 & Lab 2 & 123 & Lab 8 \\
\hline C24:0 & 38.2 & 13.8 & $36.1 \%$ & 8 & 4.87 & Lab 12 & 51.6 & Lab 4 \\
\hline C24:1n9 & 56.2 & 18.0 & $32.0 \%$ & 8 & 12.6 & Lab 12 & 71.5 & Lab 4 \\
\hline
\end{tabular}

List any additional information such as known coelutions or results for additional fatty acids below:

\begin{tabular}{|l|l|l|l|l|l|l|l|l|}
\hline C15:0 & 18.9 & 6.7 & $35.5 \%$ & 4 & 14.8 & Lab 6 & 25.7 & Lab 13 \\
\hline C17:0 & 22.6 & 7.6 & $33.8 \%$ & 5 & 21.6 & Lab 4 & 37.6 & Lab 13 \\
\hline
\end{tabular}

\begin{tabular}{|l|l|l|l|l|l|l|l|l|}
\hline density $(\mathrm{g} / \mathrm{mL})$ & 1.03 & 0.04 & $3.9 \%$ & 7 & 0.948 & Lab 6 & 1.07 & Lab 10 \\
\hline
\end{tabular}

${ }^{a} \mathrm{n}$ is number of laboratories reporting a value. 
Table 11. Laboratory 12 's data for free fatty acids $(\mu \mathrm{M})$

\begin{tabular}{|c|c|c|c|c|c|c|c|c|c|c|}
\hline & \multicolumn{3}{|c|}{ Unknown $004(n=3)$} & \multicolumn{3}{|c|}{ Unknown $005(\mathrm{n}=3)$} & \multicolumn{3}{|c|}{ Unknown $006(n=3)$} & \multirow[t]{2}{*}{ SRM $1950(n=1)$} \\
\hline & mean & stdev & rsd & mean & stdev & rsd & mean & stdev & rsd & \\
\hline C14:0 & 12.84 & 0.95 & $7.41 \%$ & 9.17 & 0.70 & $7.64 \%$ & 9.83 & 0.62 & $6.29 \%$ & 7.96 \\
\hline C14:1n5 & 2.38 & 0.21 & $8.74 \%$ & 1.83 & 0.20 & $11.15 \%$ & 1.74 & 0.18 & $10.21 \%$ & 1.60 \\
\hline C16:0 & 125.35 & 12.41 & $9.90 \%$ & 86.98 & 8.53 & $9.81 \%$ & 88.71 & 4.61 & $5.20 \%$ & 80.42 \\
\hline C16:1n7 & 15.02 & 1.65 & $10.98 \%$ & 7.60 & 0.66 & $8.65 \%$ & 8.18 & 0.26 & $3.22 \%$ & 10.38 \\
\hline C18:0 & 42.22 & 2.44 & $5.78 \%$ & 37.43 & 3.61 & $9.65 \%$ & 37.67 & 1.53 & $4.05 \%$ & 30.65 \\
\hline C18:1n7 & 7.68 & 0.62 & $8.03 \%$ & 4.79 & 0.32 & $6.59 \%$ & 5.00 & 0.21 & $4.23 \%$ & 5.55 \\
\hline C18:1n9 & 138.26 & 11.83 & $8.56 \%$ & 100.25 & 7.25 & $7.23 \%$ & 95.47 & 1.63 & $1.71 \%$ & 86.75 \\
\hline C18:2n6 & 77.85 & 6.43 & $8.26 \%$ & 54.97 & 4.41 & $8.02 \%$ & 57.81 & 2.45 & $4.23 \%$ & 47.89 \\
\hline C18:3n3 & 6.81 & 0.51 & $7.50 \%$ & 5.17 & 0.46 & $8.85 \%$ & 4.75 & 0.21 & $4.34 \%$ & 4.19 \\
\hline C18:3n6 & 1.00 & 0.03 & $2.59 \%$ & 7.83 & 12.38 & $158.25 \%$ & 0.73 & 0.04 & $5.00 \%$ & 0.70 \\
\hline C20:0 & 1.25 & 0.02 & $1.85 \%$ & 1.21 & 0.12 & $9.75 \%$ & 1.28 & 0.06 & $4.31 \%$ & 1.46 \\
\hline C20:1n9 & 2.04 & 0.04 & $1.89 \%$ & 1.76 & 0.02 & $0.89 \%$ & 1.73 & 0.04 & $2.42 \%$ & 1.91 \\
\hline $\mathrm{C} 20: 2 \mathrm{n} 6$ & 1.51 & 0.05 & $3.45 \%$ & 1.36 & 0.08 & $5.98 \%$ & 1.28 & 0.13 & $9.86 \%$ & 1.34 \\
\hline C20:3n6 & 15.41 & 0.85 & $5.50 \%$ & 13.48 & 2.07 & $15.33 \%$ & 16.46 & 1.37 & $8.33 \%$ & 10.61 \\
\hline C20:4n6 & 14.65 & 0.80 & $5.44 \%$ & 15.50 & 2.52 & $16.25 \%$ & 13.23 & 1.21 & $9.15 \%$ & 10.71 \\
\hline C20:5n3 & 0.37 & 0.01 & $3.37 \%$ & 0.34 & 0.01 & $2.95 \%$ & 0.35 & 0.02 & $4.91 \%$ & 0.31 \\
\hline C22:0 & 0.62 & 0.01 & $1.53 \%$ & 0.57 & 0.03 & $5.22 \%$ & 0.60 & 0.01 & $2.44 \%$ & 0.53 \\
\hline C22:1n9 & \multicolumn{3}{|c|}{ Not analyzed (NA) } & NA & & & NA & & & NA \\
\hline C22:4n6 & 1.06 & 0.08 & $7.14 \%$ & 0.91 & 0.04 & $4.76 \%$ & 0.99 & 0.11 & $11.52 \%$ & 1.11 \\
\hline C22:5n3 & 1.19 & 0.07 & $6.00 \%$ & 1.19 & 0.04 & $2.95 \%$ & 1.20 & 0.08 & $6.58 \%$ & 0.97 \\
\hline C22:5n6 & 1.03 & 0.06 & $5.90 \%$ & 0.91 & 0.10 & $10.61 \%$ & 0.89 & 0.04 & $4.56 \%$ & 0.93 \\
\hline C22:6n3 & 1.51 & 0.06 & $4.21 \%$ & 1.79 & 0.57 & $31.68 \%$ & 2.78 & 0.26 & $9.20 \%$ & 0.81 \\
\hline C24:0 & 0.48 & 0.03 & $6.71 \%$ & 0.42 & 0.03 & $6.10 \%$ & 0.61 & 0.08 & $13.70 \%$ & 0.43 \\
\hline C24:1n9 & 0.92 & 0.12 & $13.20 \%$ & 0.76 & 0.07 & $8.69 \%$ & 0.98 & 0.22 & $22.79 \%$ & 0.85 \\
\hline
\end{tabular}

List any additional information such as known coelutions or results for additional fatty acids below:

\begin{tabular}{|l|c|c|c||c|c|c|c|c|c||c||}
\hline $\mathrm{C} 12: 0$ & 5.80 & 0.14 & $2.41 \%$ & 4.23 & 0.26 & $6.18 \%$ & 4.90 & 0.64 & $13.08 \%$ & 3.79 \\
\hline $\mathrm{C} 15: 0$ & 2.19 & 0.14 & $6.63 \%$ & 1.96 & 0.19 & $9.63 \%$ & 2.08 & 0.10 & $4.77 \%$ & 1.54 \\
\hline $\mathrm{C} 16: 1 \mathrm{n} 7 \mathrm{t}$ & 3.00 & 0.31 & $10.49 \%$ & 2.27 & 0.18 & $8.04 \%$ & 3.77 & 2.08 & $55.20 \%$ & 2.10 \\
\hline $\mathrm{C} 17: 0$ & 2.12 & 0.12 & $5.72 \%$ & 1.89 & 0.12 & $6.36 \%$ & 1.84 & 0.05 & $2.59 \%$ & 1.60 \\
\hline $\mathrm{C} 17: 1 \mathrm{n} 9$ & 1.43 & 0.07 & $4.54 \%$ & 1.18 & 0.09 & $7.67 \%$ & 1.08 & 0.04 & $3.33 \%$ & 1.21 \\
\hline 9c,11t-CLA & 2.06 & 0.07 & $3.23 \%$ & 1.79 & 0.06 & $3.11 \%$ & 1.77 & 0.06 & $3.38 \%$ & 2.01 \\
\hline C18:4n3 & 0.24 & 0.16 & $64.90 \%$ & 0.25 & 0.15 & $60.06 \%$ & 0.31 & 0.05 & $15.88 \%$ & 0.09 \\
\hline C19:0 & 0.75 & 0.03 & $3.69 \%$ & 0.65 & 0.02 & $2.70 \%$ & 0.69 & 0.02 & $2.56 \%$ & 0.63 \\
\hline C20:3n3 & 0.77 & 0.05 & $6.54 \%$ & 0.66 & 0.01 & $1.96 \%$ & 0.73 & 0.08 & $10.87 \%$ & 0.64 \\
\hline C20:4n3 & 0.98 & 0.01 & $1.46 \%$ & 0.96 & 0.09 & $9.66 \%$ & 1.67 & 0.16 & $9.89 \%$ & 0.66 \\
\hline C21:0 & 0.41 & 0.07 & $17.98 \%$ & 0.36 & 0.03 & $9.35 \%$ & 0.47 & 0.15 & $31.37 \%$ & 0.36 \\
\hline C22:2n6 & 0.76 & 0.05 & $6.76 \%$ & 0.70 & 0.01 & $1.54 \%$ & 0.69 & 0.01 & $1.28 \%$ & 0.81 \\
\hline
\end{tabular}


Table 12. \% of free to total fatty acids based on Laboratory 12 's data

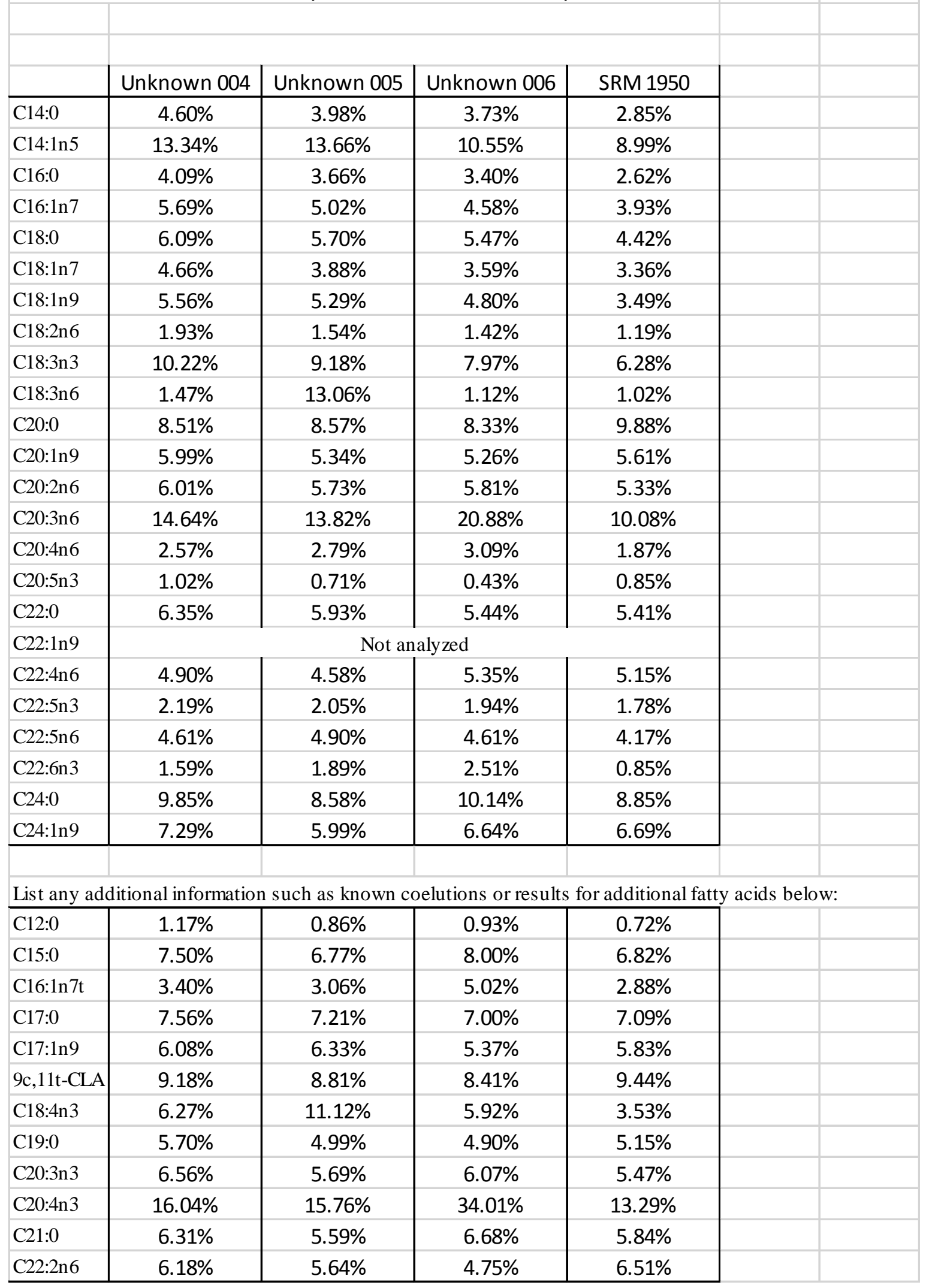


Appendix A.

Methods reported by the participating laboratories

\section{Table A-1. Procedures used}

Table A-2. Calibration information

Table A-3. Additional notes provided by laboratories 
Table A-1 Procedures used for total fatty acids

\begin{tabular}{|c|c|c|c|c|c|}
\hline Lab \# & 1 & 2 & 3 & 4 & 5 \\
\hline Reporting date & $5 / 9 / 2016$ & 5/18/2016 & 5/23/2016 & 5/25/2016 & $5 / 31 / 2016$ \\
\hline Volume of sample extracted $(\mathrm{mL}):$ & 0.50 & 0.05 & 0.20 & 0.10 & 0.02 \\
\hline \multicolumn{6}{|l|}{ Mass of samples extracted (g) (average of 3): } \\
\hline FAQAP unk004 & 0.49 & not recorded & 0.211 & 0.10 & \\
\hline FAQAP unk005 & 0.52 & not recorded & 0.210 & 0.10 & \\
\hline FAQAP unk006 & 0.48 & not recorded & 0.206 & 0.10 & \\
\hline \multicolumn{6}{|l|}{ Mass of samples extracted (g): } \\
\hline SRM 1950 & 0.51 & not recorded & 0.208 & 0.10 & \\
\hline Analytical method reference, if available & $\begin{array}{l}\text { Sánchez-Ávila N, Mata-Granados JM, } \\
\text { Ruiz-Jiménez J, Luque de Castro MD, J } \\
\text { Chromatog A } 1216 \text { (2009) 6864-6872 }\end{array}$ & $\begin{array}{l}\text { Lagerstedt SA, Hinrichs DR, Batt SM, } \\
\text { Magera MJ, Rinaldo P, McConnell JP. } \\
\text { (2001) Quantitative determination of } \\
\text { plasma C8-C26 total fatty acids for the } \\
\text { biochemical diagnosis of nutritional } \\
\text { and metabolic disorders. Mol Genet } \\
\text { Metab,.73:38-45. }\end{array}$ & & $\begin{array}{l}\text { Lagerstedt SA, Hinrichs DR, Batt SM, } \\
\text { Magera MJ, Rinaldo P, McConnell JP. } \\
\text { Quantitative determination of plasma } \\
\text { C8-C26 total fatty acids for the } \\
\text { biochemical liagnosis of nutritional } \\
\text { and metabolic disorders. Mol Genet } \\
\text { Metab 2001;73:38-45 }\end{array}$ & $\begin{array}{l}\text { Bagga D, Capone S, Wang HJ, Heber } \\
\text { D, Lill M, Chap L, Glasphy JA. J Nat } \\
\text { Cancer Ins 1997; 89:1123-1131. }\end{array}$ \\
\hline \multicolumn{6}{|c|}{\begin{tabular}{l|l} 
Analytical method used: &
\end{tabular}} \\
\hline Hydrolysis method & $\begin{array}{c}0.4 \mathrm{M} \mathrm{KOH} \text { methanol followed by } 1 \mathrm{M} \\
\mathrm{H}_{2} \mathrm{SO}_{4} \text { methanol }\end{array}$ & Acid and Base Hydrolysis & Acetic acid with Methanol & $\begin{array}{l}\text { Acid hydrolysis followed by basic } \\
\text { hydrolysis and re-acidification }\end{array}$ & $\begin{array}{l}\text { Add sample to } 1 \mathrm{ml} \text { methanol:benzene } \\
\text { (4:1). Add } 100 \text { ul acetyl chloride. Heat } \\
\text { for } 60 \text { minutes at } 100 \mathrm{C} \text {. }\end{array}$ \\
\hline Extraction method & Liquid-liquid & Liquid Liquid Extraction (LLE) & Liquid/Liquid Extraction & liquid-liquid & \\
\hline Extraction solvent & hexane with $40 \mathrm{mg} / \mathrm{g}$ BHT & Hexane & Hexane & Hexane & \\
\hline Extraction time & vortex 30 s each time & $2 \min$ & $\begin{array}{c}14 \text { per batch; each batch about } 8 \text { hours } \\
\text { for extraction }\end{array}$ & 20 min on rotary mixer & \\
\hline Extraction - other details & $\begin{array}{l}\text { Hexane extraction performed } 2 x \\
\text { following base addition and } 2 x \\
\text { following acid addition }\end{array}$ & & & $\begin{array}{l}\text { Extraction solvent evaporated, samples } \\
\text { derivatized then reconstituted in } \\
\text { hexane }+0.5 \mathrm{~N} \mathrm{HCl}\end{array}$ & $\begin{array}{l}\text { Cool sample to room } \mathrm{T} \text { and add } 4 \mathrm{ml} \text { of } \\
\text { potassium carbonate }(6 \%) \text {. Centerifuge } \\
\text { and inject supernatant into GC. }\end{array}$ \\
\hline Sample extract cleanup method & none & Hexane LLE from derivatizing reagent & None & $\begin{array}{l}\text { Centrifugation of samples and removal } \\
\text { of supernatant for injection }\end{array}$ & \\
\hline Derivitization reagent & acidic methanol & pentafluorobenzylbromide & $\begin{array}{l}\text { Methyl ester is formed from the } \\
\text { methanol }\end{array}$ & $\begin{array}{l}\text { 2,3,4,5,6-Pentaflourobenzyl bromide } \\
\text { (10\% solution in acetonitrile) }\end{array}$ & \\
\hline Analytical instrument & GC-FID & $\begin{array}{c}\text { 6890N/5973N Gas } \\
\text { Chromatograph/Mass Spectrometer }\end{array}$ & Agilent MSD 5973N, Agilent GC 6890N & $\begin{array}{l}\text { Agilent } 6890 \text { GC/ } 5975 \mathrm{C} \text { CI MSD in } \\
\text { negative ion mode }\end{array}$ & $\begin{array}{l}\text { Agilent Technologies } 5890 \mathrm{~A} \text { gas } \\
\text { chromatograph }\end{array}$ \\
\hline Column phase & $\begin{array}{l}\text { crossbonded polyethylene glycol } \\
\text { (FAMEWAX, Restek) }\end{array}$ & 5\% phenyl-95\% methylpolysiloxane & DB-23 & DB-5MS & $\begin{array}{l}\text { SP2380 stabilized phase fused silica } \\
\text { capillary column (Sigma-Aldrich) }\end{array}$ \\
\hline Column length, $\mathrm{m}$ & 30 & 20 & 60 & $30 m+10 m$ guard & 30 \\
\hline Column i.d., mm & 0.32 & 0.18 & 0.25 & 0.25 & 0.32 \\
\hline Column film thickness, $\mu \mathrm{m}$ & 0.25 & 0.18 & 0.15 & 0.25 & 0.20 \\
\hline Injection method (split, splitless, etc) & split & split and splitless & Split & $\begin{array}{l}\text { Split and splitless injections for } \\
\text { different fatty acids }\end{array}$ & split injection \\
\hline \multicolumn{6}{|l|}{ Method of quantitation: } \\
\hline $\mathrm{ES}=$ external standards $(\mathrm{Y} / \mathrm{N})$ & & $\mathrm{N}$ & $\mathrm{N}$ & $\mathrm{Y}$ & $\mathrm{Y}$ \\
\hline Number of ES used & & 0 & & 37 compounds at 6 levels each & 12 \\
\hline IS = internal standards $(\mathrm{Y} / \mathrm{N})$ & yes & $\mathrm{Y}$ & $\mathrm{Y}$ & $\mathrm{Y}$ & \\
\hline Number of IS used & 2 & 13 & 4 & 13 in one solution & \\
\hline ed PRIOR to & yes & $\mathrm{Y}$ & $\mathrm{Y}$ & $\mathrm{Y}$ & \\
\hline
\end{tabular}




\begin{tabular}{|c|c|c|c|c|c|}
\hline Table A-1 Continued & & & & & \\
\hline Lab \# & 6 & 7 & 8 & 9 & 10 \\
\hline Reporting date & "5/31/2016 & 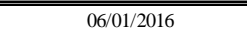 & "6/1/2016 & (6/1/2016 & 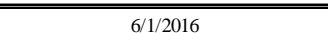 \\
\hline Volume of sample extracted (mL): & 0.10 & 0.0031 & 0.10 & 0.10 & 0.05 \\
\hline \multicolumn{6}{|l|}{ Mass of samples extracted (g) (average of 3): } \\
\hline FAQAP unk004 & 0.096 & & 0.103 & 0.10 & 0.052 \\
\hline FAQAP unk005 & 0.097 & & 0.104 & 0.10 & 0.055 \\
\hline FAQAP unk006 & 0.095 & & 0.103 & 0.10 & 0.054 \\
\hline \multicolumn{6}{|l|}{ Mass of samples extracted (g): } \\
\hline SRM 1950 & 0.095 & & 0.103 & 0.10 & 0.054 \\
\hline Analytical method reference, if available & $\begin{array}{l}\text { Lagertedt S, Hinrichs D, Batt S, Magera } \\
\text { M, Rinaldo P, McConnell J. } \\
\text { Quantitative Determination of Plasma } \\
\text { C8-C26 Total Fatty Acids for the } \\
\text { Biochemical Diagnosis of Nutritional } \\
\text { and Metabolic Disorders. Mol Genet \& } \\
\text { Metab 2001; 73: 38-45. }\end{array}$ & & & & \\
\hline \multicolumn{6}{|c|}{\begin{tabular}{l|l|} 
Analytical method used: & \\
\end{tabular}} \\
\hline Hydrolysis method & Acid/Base & & & $\begin{array}{c}\text { Acidic and alkaline hydrolysis using } \\
10 \% 6 \mathrm{~N} \mathrm{HCl} \text { in acetonitrile and } 10 \% \\
10 \mathrm{~N} \mathrm{NaOH} \text { in MeOH }\end{array}$ & \\
\hline Extraction method & Liquid-liquid & Liquid extraction & Liquid-liquid Extraction & Liquid-liquid extraction & Modified Folch \\
\hline Extraction solvent & hexane & $0.001 \%$ BHT in Methanol & Chloroform-Methanol (2:1) & Hexanes & 3mL 2:1 chloroformmethanol \\
\hline Extraction time & 20-30 minutes per extraction & At 300 rpm for 15 Minutes & $3 \mathrm{~min}$ & 15 minutes & 24 hours in 2:1 chloroformmethanol \\
\hline Extraction - other details & $\begin{array}{l}\text { triple extraction via automated liquid } \\
\text { handler }\end{array}$ & Follow by $90 \%$ Acetonitrile & & samples extracted $3 \mathrm{x}$ & $\begin{array}{l}\text { Addition of 500uL sodium phosphate } \\
\text { buffer, vortex, centrifuge, double } \\
\text { extraction of organic phase }\end{array}$ \\
\hline Sample extract cleanup method & Speedvac & & & $\begin{array}{l}\text { Solvent evaporated and samples } \\
\text { derivatized }\end{array}$ & Hexane/water \\
\hline Derivitization reagent & PFBBr - pentafluorobenzyl bromide & & $\begin{array}{l}\text { METH-PREP II, Grace Davision } \\
\text { Discovery Sciences }\end{array}$ & pentafluorobenzyl-bromide & $14 \%$ boron trifluoride in methanol \\
\hline Analytical instrument & GC/MS & LC-MS/MS & Agilent 7890A GC, 5975C MSD & Agilent 7890/5975B GC-MS & Varian 3900 GC-FID \\
\hline Column phase & $\begin{array}{c}90 \% \text { biscyanopropyl/10\% } \\
\text { phenylcyanopropyl polysiloxane }\end{array}$ & $\mathrm{C} 18$ & $\begin{array}{l}\text { Non bonded, Poly ( } \\
\text { 80\%biscyanopropyl/20\%cyanopropyl } \\
\text { phenyl siloxane) phase }\end{array}$ & Select-FAME & DB-FFAP \\
\hline Column length, $\mathrm{m}$ & 60 & 0.005 & 30 & 200 & 15 \\
\hline Column i.d., mm & 0.25 & 2.1 & 0.25 & 0.25 & 0.10 \\
\hline Column film thickness, $\mu \mathrm{m}$ & 0.2 & 3 & 0.20 & 0.25 & 0.10 \\
\hline Injection method (split, splitless, etc) & split & & splitless & split 20:1 & 50:1 split ratio \\
\hline \multicolumn{6}{|l|}{ Method of quantitation: } \\
\hline $\mathrm{ES}=$ external standards $(\mathrm{Y} / \mathrm{N})$ & $\mathrm{N}$ & $\mathrm{Y}$ & $\mathrm{N}$ & $\mathrm{N}$ & $\mathrm{Y}$ \\
\hline Number of ES used & & 3 & & & 28 \\
\hline IS = internal standards $(\mathrm{Y} / \mathrm{N})$ & $\mathrm{Y}$ & $\mathrm{Y}$ & $\mathrm{Y}$ & $\mathrm{Y}$ & $\mathrm{Y}$ \\
\hline Number of IS used & 17 & 1 & 2 & 16 & 1 \\
\hline IS added PRIOR to extraction of sample & $\mathrm{Y}$ & $\mathrm{Y}$ & $\mathrm{Y}$ & $\mathrm{Y}$ & $\mathrm{Y}$ \\
\hline
\end{tabular}


Table A-1 Continued

\begin{tabular}{|c|c|c|c|}
\hline Lab \# & 11 & 12 & 13 \\
\hline Reporting date & $6 / 8 / 2016$ & $6 / 23 / 2016$ & $\begin{array}{ll}6 / 29 / 2016 \\
\end{array}$ \\
\hline Volume of sample extracted (mL): & 0.15 & 0.05 & 0.20 \\
\hline \multicolumn{4}{|l|}{ Mass of samples extracted (g) (average of 3): } \\
\hline FAQAP unk004 & 0.152 & & 0.21 \\
\hline FAQAP unk005 & 0.153 & & 0.21 \\
\hline FAQAP unk006 & 0.148 & & 0.21 \\
\hline \multicolumn{4}{|l|}{ Mass of samples extracted (g): } \\
\hline SRM 1950 & 0.152 & & 0.21 \\
\hline \multicolumn{3}{|l|}{ Analytical method reference, if available: } & Liu et al 2010 \\
\hline \multicolumn{4}{|l|}{ Analytical method used: } \\
\hline Hydrolysis method & Acid-catalyzed transesterification & $\begin{array}{c}0.5 \mathrm{M} \text { dry methanolic sodium methoxide } \\
\text { (as part of derivatization) }\end{array}$ & \\
\hline Extraction method & Organic Phase with Vortexing & $\begin{array}{c}\text { Based on F. Smedes, Analyst, 1999,124, } \\
\text { 1711-1718 }\end{array}$ & Solvent extraction \\
\hline Extraction solvent & 3:2 Hexane-Isopropanol & $\begin{array}{c}\text { 0.41mL 2-propanol, } 0.52 \mathrm{~mL} \\
\text { cyclohexane, } 0.57 \mathrm{~mL} 0.1 \mathrm{M} \text { ammonium } \\
\text { acetate }\end{array}$ & Hexane:2-proponol (3:2, v/v) \\
\hline Extraction time & 10 minutes & 6 minutes & \\
\hline Extraction - other details & & $\begin{array}{l}\text { Transfer cyclohexane fraction and re- } \\
\text { extract with added } 0.57 \mathrm{~mL} \text { cyclohexane }\end{array}$ & \\
\hline Sample extract cleanup method & $\begin{array}{l}\text { One wash in Hexane/dd-water and } \\
\text { wash in dd-water }\end{array}$ & $\begin{array}{l}\text { Evaporate to dryness and reconstitute } \\
\text { in 1:1 methano/toluene }\end{array}$ & \\
\hline Derivitization reagent & 4\% Sulfuric Acid in Methanol & $\begin{array}{c}0.5 \mathrm{M} \text { dry methanolic sodium } \\
\text { methoxide, } 600 \mathrm{C} 60 \mathrm{~min},+0.1 \mathrm{~mL} 3 \mathrm{~N} \\
\text { Methanolic } \mathrm{HCl}, 60^{\circ} \mathrm{C} 30 \text { min, } \\
\text { neutralize, extract with } 0.4 \mathrm{~mL} \text { hexanes }\end{array}$ & \\
\hline Analytical instrument & $\begin{array}{l}\text { Hewlett-Packard Model (now Agilent) } \\
\text { GC } 6890 \text { FID gas chromatograph with } \\
7673 \text { Autosampler injector }\end{array}$ & $\begin{array}{l}\text { Agilent } 6890 \text { Plus+ GC, 5973N MSD, } \\
7683 \text { Injector, DB-225ms column }\end{array}$ & Thermo Trace GC-DSQ system \\
\hline Column phase & $\begin{array}{l}\text { fused silica capillary cis/trans column } \\
\text { SP2560 }\end{array}$ & $\begin{array}{l}\text { (50\%-Cyanopropylphenyl)- } \\
\text { dimethylpolysiloxane }\end{array}$ & $\begin{array}{l}\text { Rxi-5MS-coated } \\
\text { 5\%dipheny/95\%dimethyl polysiloxane } \\
\text { capillary column }\end{array}$ \\
\hline Column length, $\mathrm{m}$ & 100 & 30 & 30.00 \\
\hline Column i.d., mm & 0.25 & 0.25 & 0.25 \\
\hline Column film thickness, $\mu \mathrm{m}$ & 0.20 & 0.25 & 0.25 \\
\hline Injection method (split, splitless, etc) & splitless & splitless & splitless \\
\hline \multicolumn{4}{|l|}{ Method of quantitation: } \\
\hline $\mathrm{ES}=$ external standards $(\mathrm{Y} / \mathrm{N})$ & $\begin{array}{l}\text { Y (NOTE: Used for identification not } \\
\text { quantification) }\end{array}$ & $\mathrm{N}$ & $\mathrm{N}$ \\
\hline Number of ES used & 43 & 0 & \\
\hline IS = internal standards $(\mathrm{Y} / \mathrm{N})$ & $\mathrm{N}$ & $\mathrm{Y}$ & $\mathrm{Y}$ \\
\hline Number of IS used & N/A & 1 & Methyl tridecanoate \\
\hline \multicolumn{2}{|l|}{ traction of sample } & $\mathrm{Y}$ & $\mathrm{Y}$ \\
\hline
\end{tabular}




\begin{tabular}{|c|c|}
\hline Lab \# & 12 \\
\hline Reporting date & $6 / 23 / 2016$ \\
\hline Volume of sample extracted $(\mathrm{mL}):$ & 0.05 \\
\hline \multicolumn{2}{|l|}{ Mass of samples extracted (g) (average of 3): } \\
\hline \multicolumn{2}{|l|}{ FAQAP unk004 } \\
\hline \multicolumn{2}{|l|}{ FAQAP unk005 } \\
\hline \multicolumn{2}{|l|}{ FAQAP unk006 } \\
\hline \multicolumn{2}{|l|}{ Mass of samples extracted (g): } \\
\hline \multicolumn{2}{|l|}{ SRM 1950} \\
\hline \multicolumn{2}{|l|}{ Analytical method reference, if available: } \\
\hline \multicolumn{2}{|l|}{ Analytical method used: } \\
\hline Extraction method & $\begin{array}{l}\text { Based on F. Smedes, Analyst, 1999,124, } \\
\text { 1711-1718 }\end{array}$ \\
\hline Extraction solvent & $\begin{array}{c}0.41 \mathrm{~mL} \text { 2-propanol, } 0.52 \mathrm{~mL} \\
\text { cyclohexane, } 0.57 \mathrm{~mL} 0.1 \mathrm{M} \text { ammonium } \\
\text { acetate }\end{array}$ \\
\hline Extraction time & 6 minutes \\
\hline Extraction - other details & $\begin{array}{l}\text { Transfer cyclohexane fraction and re- } \\
\text { extract with added } 0.57 \mathrm{~mL} \text { cyclohexane }\end{array}$ \\
\hline Sample extract cleanup method & $\begin{array}{l}\text { Evaporate to dryness and reconstitute } \\
\text { in 1:1 methanol/toluene }\end{array}$ \\
\hline Derivitization reagent & $\begin{array}{l}\text { 2M Tri(methylsilyl)diazomethane (in } \\
\text { hexanes) }\end{array}$ \\
\hline Analytical instrument & $\begin{array}{l}\text { Agilent } 6890 \text { Plus + GC, 5973N MSD, } \\
7683 \text { Injector, DB-225ms column }\end{array}$ \\
\hline Column phase & $\begin{array}{l}\text { (50\%-Cyanopropylphenyl)- } \\
\text { dimethylpolysiloxane }\end{array}$ \\
\hline Column length, $\mathrm{m}$ & 30 \\
\hline Column i.d., $\mathrm{mm}$ & 0.25 \\
\hline Column film thickness, $\mu \mathrm{m}$ & 0.25 \\
\hline Injection method (split, splitless, etc) & splitless \\
\hline \multicolumn{2}{|l|}{ Method of quantitation: } \\
\hline ES = external standards $(\mathrm{Y} / \mathrm{N})$ & $\mathrm{N}$ \\
\hline Number of ES used & 0 \\
\hline IS = internal standards $(\mathrm{Y} / \mathrm{N})$ & $\mathrm{Y}$ \\
\hline Number of IS used & 1 \\
\hline IS added PRIOR to extraction of sample & $\mathrm{Y}$ \\
\hline
\end{tabular}


Table A-3 Calibration information

\begin{tabular}{|c|c|c|c|c|c|c|c|c|c|}
\hline Lab & \begin{tabular}{|l} 
Fatty Acid \\
Code \\
\end{tabular} & Fatty Acid Name & \begin{tabular}{|c}
$\begin{array}{c}\text { Lowest std } \\
(\mu \mathrm{mol} / \mathrm{L})\end{array}$ \\
\end{tabular} & $\begin{array}{c}\text { Highest std } \\
(\mu \mathrm{mol} / \mathrm{L})\end{array}$ & $\begin{array}{c}\text { LOD } \\
(\mu \mathrm{mol} / \mathrm{L})\end{array}$ & $\begin{array}{c}\text { LOQ } \\
(\mu \mathrm{mol} / \mathrm{L})\end{array}$ & Curve Type & Weighting & IS used for quantitation \\
\hline 1 & C14:0 & Myristic acid & \multicolumn{6}{|c|}{ used response factors based on three independently prepared calibration solutoins } & Myristic- $\mathrm{d}_{27}$ \\
\hline & C14:1n5 & Myristoleic acid & \multicolumn{6}{|c|}{ used response factors based on three independently prepared calibration solutoins } & Myristic- $\mathrm{d}_{27}$ \\
\hline & C16:0 & Palmitic acid & \multicolumn{6}{|c|}{ used response factors based on three independently prepared calibration solutoins } & Palmitic-d $\mathrm{d}_{31}$ \\
\hline & C16:1n7 & Palmitoleic acid & \multicolumn{6}{|c|}{ used response factors based on three independently prepared calibration solutoins } & Palmitic- $\mathrm{d}_{31}$ \\
\hline & C18:0 & Stearic acid & \multicolumn{6}{|c|}{ used response factors based on three independently prepared calibration solutoins } & Palmitic- $\mathrm{d}_{31}$ \\
\hline & C18:1n7 & cis-Vaccenic acid & \multicolumn{6}{|c|}{ used response factors based on three independently prepared calibration solutoins } & Palmitic- $\mathrm{d}_{31}$ \\
\hline & C18:1n9 & Oleic acid & \multicolumn{6}{|c|}{ used response factors based on three independently prepared calibration solutoins } & Palmitic- $\mathrm{d}_{31}$ \\
\hline & C18:2n6 & Linoleic acid & \multicolumn{6}{|c|}{ used response factors based on three independently prepared calibration solutoins } & Palmitic- $\mathrm{d}_{31}$ \\
\hline & C18:3n3 & alpha-Linolenic acid & \multicolumn{6}{|c|}{ used response factors based on three independently prepared calibration solutoins } & Palmitic- $\mathrm{d}_{31}$ \\
\hline & C18:3n6 & gamma-Linolenic acid & \multicolumn{6}{|c|}{ used response factors based on three independently prepared calibration solutoins } & Palmitic- $\mathrm{d}_{31}$ \\
\hline & C20:0 & Arachidic acid & \multicolumn{6}{|c|}{ used response factors based on three independently prepared calibration solutoins } & Palmitic- $\mathrm{d}_{31}$ \\
\hline & C20:1n9 & 11-Eicosenoic acid & \multicolumn{6}{|c|}{ used response factors based on three independently prepared calibration solutoins } & Palmitic- $\mathrm{d}_{31}$ \\
\hline & C20:4n6 & Arachidonic acid & \multicolumn{6}{|c|}{ used response factors based on three independently prepared calibration solutoins } & Palmitic- $\mathrm{d}_{31}$ \\
\hline & C20:5n3 & Eicosapentaenoic acid & \multicolumn{6}{|c|}{ used response factors based on three independently prepared calibration solutoins } & Palmitic- $\mathrm{d}_{31}$ \\
\hline & C22:0 & Docosanoic acid & used respons & e factors base & on three in & pendently $\mathrm{P}$ & epared calibrat & n solutoins & Palmitic- $\mathrm{d}_{31}$ \\
\hline & C22:1n9 & Docosenoic acid & used respons & e factors base & on three in & pendently $\mathrm{p}$ & epared calibrat & n solutoins & Palmitic- $\mathrm{d}_{31}$ \\
\hline & C22:5n3 & Docosapentaenoic acid & used respons & e factors base & on three in & pendently $\mathrm{P}$ & epared calibrat & n solutoins & Palmitic- $\mathrm{d}_{31}$ \\
\hline & C22:6n3 & Docosahexaenoic acid & used respons & e factors base & on three in & pendently $\mathrm{P}$ & epared calibrat & n solutoins & Palmitic- $\mathrm{d}_{31}$ \\
\hline & C24:0 & Lignoceric acid & used respons & e factors base & on three in & pendently $\mathrm{P}$ & epared calibrat & n solutoins & Palmitic- $\mathrm{d}_{31}$ \\
\hline & C24:1n9 & Nervonic acid & used respons & e factors base & on three in & pendently p & epared calibrat & n solutoins & Palmitic- $\mathrm{d}_{31}$ \\
\hline 2 & C14:0 & Myristic acid & 12.5 & 150 & 0.3 & 3000 & Linear & None & Myristic acid- $\mathrm{d}_{3}$ \\
\hline & C14:1n5 & Myristoleic acid & 1.25 & 15 & 0.013 & 300 & Linear & None & Pentadecanoic acid- $\mathrm{d}_{4}$ \\
\hline & C16:0 & Palmitic acid & 25 & 300 & 2.81 & 6000 & Linear & None & Palmitic- $\mathrm{d}_{3}$ \\
\hline & C16:1n7 & Palmitoleic acid & 12.5 & 150 & 0.045 & 3000 & Linear & None & Myristic acid- $\mathrm{d}_{3}$ \\
\hline & C18:0 & Stearic acid & 25 & 300 & 3.719 & 6000 & Linear & None & Stearic acid- $\mathrm{d}_{3}$ \\
\hline & C18:1n7 & cis-Vaccenic acid & 25 & 300 & 0.089 & 6000 & Linear & None & Stearic acid- $\mathrm{d}_{4}$ \\
\hline & C18:1n9 & Oleic acid & 25 & 300 & 0.21 & 6000 & Linear & None & Stearic acid-d $\mathrm{d}_{5}$ \\
\hline & C18:2n6 & Linoleic acid & 25 & 300 & 0.15 & 6000 & Linear & None & Stearic acid-d 6 \\
\hline & C18:3n3 & alpha-Linolenic acid & 12.5 & 150 & 0.009 & 3000 & Linear & None & Arachidic acid- $\mathrm{d}_{3}$ \\
\hline & C18:3n6 & gamma-Linolenic acid & 12.5 & 150 & 0.009 & 3000 & Linear & None & Arachidic acid-d $\mathrm{d}_{3}$ \\
\hline & C20:0 & Arachidic acid & 12.5 & 150 & 0.25 & 3000 & Linear & None & Arachidic acid- $\mathrm{d}_{3}$ \\
\hline & C20:3n6 & homo-gamma-Linolenic acid & 0.625 & 7.5 & 0.004 & 150 & Linear & None & Docosanoic acid- $\mathrm{d}_{4}$ \\
\hline & C20:4n6 & Arachidonic acid & 12.5 & 150 & 0.021 & 3000 & Linear & None & Arachidic acid-d $\mathrm{d}_{3}$ \\
\hline & C20:5n3 & Eicosapentaenoic acid & 12.5 & 150 & 0.019 & 3000 & Linear & None & Arachidic acid- $\mathrm{d}_{3}$ \\
\hline & C22:0 & Docosanoic acid & 0.625 & 7.5 & 0.036 & 150 & Linear & None & Docosanoic acid- $\mathrm{d}_{4}$ \\
\hline & C22:4n6 & Docosatetraenoic acid & 0.625 & 7.5 & 0.002 & 150 & Linear & None & Docosanoic acid- $\mathrm{d}_{4}$ \\
\hline & C22:5n3 & Docosapentaenoic acid & 0.625 & 7.5 & 0.021 & 150 & Linear & None & Docosanoic acid- $\mathrm{d}_{4}$ \\
\hline & C22:5n6 & Docosapentaenoic acid & 0.625 & 7.5 & 0.021 & 150 & Linear & None & Docosanoic acid- $\mathrm{d}_{4}$ \\
\hline & C22:6n3 & Docosahexaenoic acid & 12.5 & 150 & 0.03 & 3000 & Linear & None & Arachidic acid-d $\mathrm{d}_{3}$ \\
\hline & C24:0 & Lignoceric acid & 0.625 & 7.5 & 0.029 & 150 & Linear & None & Tetracosanoic acid-d $\mathrm{d}_{4}$ \\
\hline & C24:1n9 & Nervonic acid & 0.625 & 7.5 & 0.004 & 150 & Linear & None & Tetracosanoic acid-d $\mathrm{d}_{4}$ \\
\hline 3 & C14:0 & myristic & 12.7 & 845 & 0.963 & 13.1 & quadratic & $1 / x$ & Methyl Tridecenoate \\
\hline & C16:0 & palmitic & 111 & 7430 & 29 & 234 & quadratic & $1 / x$ & Methyl trans-Heptadecenoate \\
\hline & C16:1 & palmitoleic & 11.4 & 757 & 3.69 & 23.6 & quadratic & $1 / x$ & Methyl Tridecenoate \\
\hline & C18:0 & stearic & 100 & 6700 & 6.22 & 105 & quadratic & $1 / x$ & Methyl trans-Heptadecenoate \\
\hline & C18:1n7 & vaccenic & 10.2 & 682 & 2.8 & 21.2 & quadratic & $1 / x$ & Methyl Heneicosanoate \\
\hline & C18:1n9 & oleic & 102 & 6790 & 36.3 & 212 & quadratic & $1 / x$ & Methyl trans-Heptadecenoate \\
\hline & C18:2n6 & linoleic & 103 & 6840 & 26 & 214 & quadratic & $1 / x$ & Methyl trans-Heptadecenoate \\
\hline & C18:3n3 & alpha-linolenic & 10.4 & 691 & 0.718 & 10.8 & quadratic & $1 / x$ & Methyl Heneicosanoate \\
\hline & C18:3n6 & gamma-linolenic & 10.4 & 690 & 0.682 & 10.8 & quadratic & $1 / \mathrm{x}$ & Methyl Heneicosanoate \\
\hline & C20:3n6 & homo-gamma-linolenic & 9.41 & 628 & 2.19 & 19.6 & quadratic & $1 / x$ & Methyl Heneicosanoate \\
\hline & C20:4n6 & arachidonic & 93.5 & 6230 & 5.12 & 98.5 & quadratic & $1 / x$ & Methyl trans-Heptadecenoate \\
\hline & C20:5n3 & eicosapentaenoic & 9.53 & 635 & 0.794 & 9.92 & quadratic & $1 / \mathrm{x}$ & Methyl Tricosenoate \\
\hline & C22:0 & docosanoic & 8.5 & 566 & 0.382 & 8.81 & quadratic & $1 / x$ & Methyl Tricosenoate \\
\hline & C22:4n6 & docosatetraenoic & 8.67 & 578 & 0.481 & 9.02 & quadratic & $1 / \mathrm{x}$ & Methyl Tricosenoate \\
\hline & C22:5n3 & docosapentaenoic & 8.72 & 581 & 0.424 & 9.08 & quadratic & $1 / \mathrm{x}$ & Methyl Tricosenoate \\
\hline & C22:6n3 & docosahexaenoic & 8.79 & 586 & 1.46 & 18.3 & quadratic & $1 / x$ & Methyl Tricosenoate \\
\hline & C24:0 & lignoceric & 7.83 & 522 & 0.353 & 8.14 & quadratic & $1 / x$ & Methyl Tricosenoate \\
\hline & C24:1n9 & nervonic & 7.87 & 525 & 0.409 & 8.18 & quadratic & $1 / \mathrm{x}$ & Methyl Tricosenoate \\
\hline
\end{tabular}


Table A-3 (cont) Calibration information

\begin{tabular}{|c|c|c|c|c|c|c|c|c|c|}
\hline Lab & \begin{tabular}{|l} 
Fatty Acid \\
Code
\end{tabular} & Fatty Acid Name & $\begin{array}{c}\text { Lowest std } \\
(\mu \mathrm{mol} / \mathrm{L})\end{array}$ & $\begin{array}{c}\text { Highest std } \\
(\mu \mathrm{mol} / \mathrm{L})\end{array}$ & $\begin{array}{c}\text { LOD } \\
(\mu \mathrm{mol} / \mathrm{L})\end{array}$ & $\begin{array}{c}\mathrm{LOQ} \\
(\mu \mathrm{mol} / \mathrm{L})\end{array}$ & Curve Type & Weighting & IS used for quantitation \\
\hline \multirow[t]{37}{*}{4} & C8:0 & Octanoic acid & 14 & 120 & & & quadratic & equal & C8:0 D3 \\
\hline & C10:0 & Decanoic acid & 13 & 110 & & & quadratic & equal & C10:0 D3 \\
\hline & C10:1 & 9-Decenoic acid & 16 & 120 & & & quadratic & equal & C10:0 D3 \\
\hline & C12:0 & Lauric acid & 12 & 101 & & & quadratic & equal & C12:0 D3 \\
\hline & C12:1w1 & 11-Dodecenoic acid & 13 & 108 & & & quadratic & equal & C12:0 D3 \\
\hline & C12:1w7 & cis-5-Dodecenoic acid & 14 & 115 & & & quadratic & equal & C12:0 D3 \\
\hline & C14:0 & Myristic acid & 44 & 365 & & & quadratic & equal & C14:0 D3 \\
\hline & C14:1 & Myristoleic acid & 14 & 115 & & & quadratic & equal & C15:0 D3 \\
\hline & C15:0 & Pentadecanoic acid & 23 & 190 & & & quadratic & equal & C15:0 D3 \\
\hline & C16:0 & Palmitic acid & 468 & 3900 & & & quadratic & equal & C16:0 D3 \\
\hline & C16:1w7 & Palmitoleic acid & 127 & 1058 & & & quadratic & equal & C14:0 D3 \\
\hline & C17:0 & Heptadecanoic acid & 22 & 180 & & & quadratic & equal & C15:0 D3 \\
\hline & C18:0 & Stearic acid & 89 & 745 & & & quadratic & equal & C18:0 D3 \\
\hline & C18:1w7 & Vaccenic acid & 241 & 2010 & & & quadratic & equal & C18:0 D3 \\
\hline & C18:1w9 & Oleic acid & 600 & 5000 & & & quadratic & equal & C18:0 D3 \\
\hline & C18:2w6 & Linoleic acid & 474 & 3950 & & & quadratic & equal & C18:0 D3 \\
\hline & C18:3w3 & a-linolenic acid & 55 & 460 & & & quadratic & equal & C18:0 D3 \\
\hline & C18:3w6 & g-linolenic acid & 60 & 500 & & & quadratic & equal & C18:0 D3 \\
\hline & C19:0 & Nonadecanoic acid & 5 & 43 & & & quadratic & equal & C22:0 D3 \\
\hline & C20:0 & Arachidic acid & 115 & 960 & & & quadratic & equal & C20:0 D3 \\
\hline & C20:3w6 & Eicosatrienoic acid (w6) (Homo-g- linolenic acid) & 13 & 112 & & & quadratic & equal & C22:0 D3 \\
\hline & C20:3w9 & 5,8,11-Eicosatrienoic acid (MEAD) & 7 & 55 & & & quadratic & equal & C22:0 D3 \\
\hline & C20:4w6 & Arachidonic acid & 121 & 1000 & & & quadratic & equal & C20:0 D3 \\
\hline & C20:5w3 & Eicosapentaenoic acid (w3) & 78 & 650 & & & quadratic & equal & C20:0 D3 \\
\hline & C21:0 & Heneicosanoic acid & 11 & 95 & & & quadratic & equal & C22:0 D3 \\
\hline & C22:0 & Docosanoic acid (Behenic acid) & 14 & 115 & & & quadratic & equal & C22:0 D3 \\
\hline & C22:1w9 & Erucic acid (w9) & 9 & 72 & & & quadratic & equal & C22:0 D3 \\
\hline & C22:4w6 & Docosatetraenoic acid (w6) & 6 & 46 & & & quadratic & equal & C22:0 D3 \\
\hline & C22:5w3 & Docosapentaenoic acid (w3) & 25 & 210 & & & quadratic & equal & C22:0 D3 \\
\hline & C22:5w6 & Docosapentaenoic acid & 6 & 50 & & & quadratic & equal & C22:0 D3 \\
\hline & C22:6w3 & Docosahexaenoic acid (w3) & 13 & 108 & & & quadratic & equal & C22:0 D3 \\
\hline & C23:0 & Tricosanoic acid & 12 & 97 & & & quadratic & equal & C24:0 D4 \\
\hline & $\mathrm{C} 24: 0$ & Tetracosanoic acid & 6 & 51 & & & quadratic & equal & C24:0 D4 \\
\hline & C24:1w9 & Nervonic acid (w9) & 23 & 195 & & & quadratic & equal & C24:0 D4 \\
\hline & C26:0 & Hexacosanoic acid & 1.4 & 11.3 & & & quadratic & equal & C26:0 D4 \\
\hline & PhA & Phytanic acid & 2.6 & 21.7 & & & quadratic & equal & PhA D3 \\
\hline & PrA & Pristanic acid & 0.25 & 2.05 & & & quadratic & equal & PrA D3 \\
\hline \multirow[t]{24}{*}{5} & C14:0 & Myristic acid & 56.8 & 909.9 & & & & & \\
\hline & C14:1n5 & Myristoleic acid & NA & NA & & & & & \\
\hline & C16:0 & Palmitic acid & 151.7 & 2428.3 & & & & & \\
\hline & C16:1n7 & Palmitoleic acid & 51.9 & 832.4 & & & & & \\
\hline & C18:0 & Stearic acid & 91.7 & 1467.9 & & & & & \\
\hline & C18:1n7 & cis-Vaccenic acid & NA & NA & & & & & \\
\hline & C18:1n9 & Oleic acid & 92.4 & 1479 & & & & & \\
\hline & C18:2n6 & Linoleic acid & 46.7 & 749.1 & & & & & \\
\hline & C18:3n3 & alpha-Linolenic acid & 47 & 750.5 & & & & & \\
\hline & C18:3n6 & gamma-Linolenic acid & NA & NA & & & & & \\
\hline & C20:0 & Arachidic acid & NA & NA & & & & & \\
\hline & C20:1n9 & 11-Eicosenoic acid & NA & NA & & & & & \\
\hline & C20:2n6 & 11,14-Eicosadienoic acid & 21.1 & 337.4 & & & & & \\
\hline & C20:3n6 & homo-gamma-Linolenic acid & NA & NA & & & & & \\
\hline & C20:4n6 & Arachidonic acid & 39.7 & 654.1 & & & & & \\
\hline & C20:5n3 & Eicosapentaenoic acid & 36.2 & 580.70 & & & & & \\
\hline & C22:0 & Docosanoic acid & NA & NA & & & & & \\
\hline & C22:1n9 & Docosenoic acid & NA & NA & & & & & \\
\hline & $\mathrm{C} 22: 4 \mathrm{n} 6$ & Docosatetraenoic acid & NA & NA & & & & & \\
\hline & C22:5n3 & Docosapentaenoic acid & 39.9 & 636.7 & & & & & \\
\hline & C22:5n6 & Docosapentaenoic acid & NA & NA & & & & & \\
\hline & C22:6n3 & Docosahexaenoic acid & 39.6 & 632.8 & & & & & \\
\hline & C24:0 & Lignoceric acid & NA & NA & & & & & \\
\hline & C24:1n9 & Nervonic acid & NA & NA & & & & & \\
\hline
\end{tabular}




\begin{tabular}{|c|c|c|c|c|c|c|c|c|c|}
\hline \multicolumn{3}{|c|}{ Table A-3 (cont) Calibration information } & \multirow[b]{2}{*}{$\begin{array}{c}\text { Lowest std } \\
(\mu \mathrm{mol} / \mathrm{L})\end{array}$} & \multirow[b]{2}{*}{$\begin{array}{c}\text { Highest std } \\
(\mu \mathrm{mol} / \mathrm{L})\end{array}$} & \multirow[b]{2}{*}{$\begin{array}{c}\text { LOD } \\
(\mu \mathrm{mo} / \mathrm{L})\end{array}$} & \multirow[b]{2}{*}{$\begin{array}{c}\mathrm{LOQ} \\
(\mu \mathrm{mol} / \mathrm{L})\end{array}$} & \multirow[b]{2}{*}{ Curve Type } & \multirow[b]{2}{*}{ Weighting } & \multirow[b]{2}{*}{ IS used for quantitation } \\
\hline Lab & $\begin{array}{l}\text { Fatty Acid } \\
\text { Code }\end{array}$ & Fatty Acid Name & & & & & & & \\
\hline \multirow[t]{30}{*}{6} & C18:3n3 & alpha-Linolenic acid & 6.53 & 653 & 1.54 & not available & linear & $1 / x^{2}$ & alpha -Linolenic-d14 \\
\hline & C20:0 & Arachidic acid & 0.90 & 89.5 & 0.82 & not available & linear & $1 / x^{2}$ & Arachidic-d39 \\
\hline & C20:4n6 & Arachidonic acid & 23.4 & 2337 & 7.34 & not available & linear & $1 / x^{2}$ & Arachidonic-d8 \\
\hline & C10:0 & Capric acid & 0.44 & 43.9 & 1.59 & not available & quadratic & $1 / x^{2}$ & Capric-d3 \\
\hline & C22:0 & Docosanoic acid & 1.83 & 183 & 0.68 & not available & linear & $1 / x^{2}$ & Docosanoic-d4 \\
\hline & C22:6n3 & Docosahexaenoic acid & 7.88 & 788 & 1.84 & not available & linear & $1 / x^{2}$ & Docosahexaenoic-d5 \\
\hline & C22:5n3 & Docosapentaenoic n-3 acid & 2.49 & 249 & 0.55 & not available & linear & $1 / x^{2}$ & Docosahexaenoic-d5 \\
\hline & C22:5n6 & Docosapentaenoic n-6 acid & 1.59 & 159 & 0.24 & not available & linear & $1 / x^{2}$ & Docosahexaenoic-d5 \\
\hline & $\mathrm{C} 22: 4 \mathrm{n} 6$ & Docosatetraenoic acid & 1.48 & 148 & 0.31 & not available & quadratic & $1 / x^{2}$ & Docosahexaenoic-d5 \\
\hline & C20:2n6 & 11,14-Eicosadienoic acid & 0.98 & 97.7 & 0.31 & not available & quadratic & $1 / x^{2}$ & Arachidic-d39 \\
\hline & C20:1n9 & 11-Eicosenoic acid & 1.00 & 100 & 0.87 & not available & quadratic & $1 / x^{2}$ & Arachidic-d39 \\
\hline & C20:5n3 & Eicosapentaenoic acid & 7.15 & 715 & 0.79 & not available & quadratic & $1 / x^{2}$ & Eicosapentaenoic-d5 \\
\hline & C20:3n9 & 5Z, 8Z, 11Z-Eicosatrienoic acid & 0.64 & 64.3 & 0.39 & not available & quadratic & $1 / x^{2}$ & Arachidic-d39 \\
\hline & C18:3n6 & gamma-Linolenic acid & 2.93 & 293 & 0.42 & not available & linear & $1 / x^{2}$ & alpha -Linolenic-d14 \\
\hline & C20:3n6 & homo-gamma-Linolenic acid & 4.62 & 462 & 1.14 & not available & quadratic & $1 / x^{2}$ & alpha -Linolenic-d14 \\
\hline & C12:0 & Lauric acid & 2.33 & 233 & 2.33 & not available & quadratic & $1 / x^{2}$ & Lauric-d3 \\
\hline & C24:0 & Lignoceric acid & 1.47 & 147 & 1.09 & not available & linear & $1 / x^{2}$ & Tetracosanoic-d4 \\
\hline & C18:2n6 & Linoleic acid & 82.7 & 8274 & 22.6 & not available & linear & $1 / x^{2}$ & 13C-Linoleic \\
\hline & C14:1n5 & Myristoleic acid & 1.42 & 142 & 0.29 & not available & quadratic & $1 / x^{2}$ & Myristic-d27 \\
\hline & C14:0 & Myristic acid & 10.1 & 1012 & 4.90 & not available & quadratic & $1 / x^{2}$ & Myristic-d27 \\
\hline & C17:0 & Margaric acid & 1.50 & 150 & 3.36 & not available & quadratic & $1 / x$ & Margaric-d3 \\
\hline & C24:1n9 & Nervonic acid & 1.95 & 195 & 0.69 & not available & quadratic & $1 / x^{2}$ & Tetracosanoic-d4 \\
\hline & C18:1n9 & Oleic acid & 85.0 & 8496 & 17.7 & not available & linear & $1 / x^{2}$ & 13C-Oleic \\
\hline & C15:0 & Pentadecanoic acid & 0.95 & 95.0 & 0.75 & not available & linear & $1 / x^{2}$ & Pentadecanoic-d3 \\
\hline & C16:1n7 & Palmitoleic acid & 25.1 & 2514 & 6.56 & not available & quadratic & $1 / x^{2}$ & Palmitoleic-d14 \\
\hline & C16:0 & Palmitic acid & 87.4 & 8742 & 78.1 & not available & quadratic & $1 / x^{2}$ & Palmitic-d31 \\
\hline & C18:4n3 & Stearidonic acid & 0.47 & 46.9 & 0.24 & not available & quadratic & $1 / x$ & Arachidic-d39 \\
\hline & C18:0 & Stearic acid & 25.0 & 2505 & 39.1 & not available & quadratic & $1 / x^{2}$ & Stearic-d35 \\
\hline & C23:0 & Tricosanoic acid & 0.98 & 97.6 & 0.90 & not available & linear & $1 / x^{2}$ & Docosanoic-d4 \\
\hline & C18:1n7 & cis-Vaccenic acid & 7.42 & 742 & 2.31 & not available & quadratic & $1 / x^{2}$ & 13C-Oleic \\
\hline \multirow[t]{3}{*}{7} & C20:4n6 & Arachidonic acid & 317 & 2952 & & & Linear & $1 / \mathrm{x}$ & Docosahexaenoic-d5 \\
\hline & C20:5n3 & Eicosapentaenoic acid & 12.50 & 116 & & & Linear & $1 / x$ & Docosahexaenoic-d5 \\
\hline & C22:6n3 & Docosahexaenoic acid & 38.1 & 354 & & & Linear & $1 / x$ & Docosahexaenoic-d5 \\
\hline \multirow[t]{14}{*}{8} & C14:0 & Myristic & 54.70 & 1094.7 & & & Linear & Equal & Margaric \\
\hline & C16:0 & Palmitic & 195 & 3899.7 & & & Linear & Equal & Margaric \\
\hline & C16:1n7 & Palmitoleic & 49.1 & 982.6 & & & Linear & Equal & Margaric \\
\hline & C18:0 & Stearic & 87.90 & 1757.6 & & & Linear & Equal & Margaric \\
\hline & C18:1n9 & Oleic & 177 & 3540.1 & & & Linear & Equal & Margaric \\
\hline & C18:2n6 & Linoleic & 178.3 & 3565.3 & & & Linear & Equal & Margaric \\
\hline & C18:3n3 & alpha-Linolenic & 22.40 & 448.9 & & & Linear & Equal & Margaric \\
\hline & C20:1n9 & 11-Eicosenoic & 20.10 & 402.5 & & & Linear & Equal & Margaric \\
\hline & C20:3n6 & homo-gamma-Linolenic & 20.4 & 407.8 & & & Linear & Equal & Margaric \\
\hline & C20:4n6 & Arachidonic & 82.1 & 1641.9 & & & Linear & Equal & Arachidonic-d8 \\
\hline & C20:5n3 & Eicosapentaenoic & 82.6 & 1652.8 & & & Linear & Equal & Arachidonic-d8 \\
\hline & C22:4n6 & Docosatetraenoic & 18.80 & 375.9 & & & Linear & Equal & Arachidonic-d8 \\
\hline & C22:5n3 & Docosapentaenoic & 18.9 & 378.1 & & & Linear & Equal & Arachidonic-d8 \\
\hline & C22:6n3 & Docosahexaenoic & 38.0 & 760.9 & & & Linear & Equal & Arachidonic-d8 \\
\hline
\end{tabular}




\begin{tabular}{|c|c|c|c|c|c|c|c|c|c|}
\hline \multicolumn{3}{|c|}{ Table A-3 (cont) Calibration information } & \multirow[b]{2}{*}{$\begin{array}{c}\text { Lowest std } \\
(\mu \mathrm{mol} / \mathrm{L})\end{array}$} & \multirow[b]{2}{*}{$\begin{array}{c}\text { Highest std } \\
(\mu \mathrm{mol} / \mathrm{L})\end{array}$} & \multirow[b]{2}{*}{$\begin{array}{c}\mathrm{LOD} \\
(\mu \mathrm{mol} / \mathrm{L})\end{array}$} & \multirow[b]{2}{*}{$\begin{array}{c}\mathrm{LOQ} \\
(\mu \mathrm{mol} / \mathrm{L})\end{array}$} & \multirow[b]{2}{*}{ Curve Type } & \multirow[b]{2}{*}{ Weighting } & \multirow[b]{2}{*}{ IS used for quantitation } \\
\hline Lab & $\begin{array}{l}\text { Fatty Acid } \\
\text { Code }\end{array}$ & Fatty Acid Name & & & & & & & \\
\hline 9 & C14:0 & Myristic acid & 24.3 & 608 & 0.33 & & Linear & none & Myristic-d27 \\
\hline & C14:1n5 & Myristoleic acid & 4.11 & 103 & 0.29 & & Linear & none & Myristic-d27 \\
\hline & C16:0 & Palmitic acid & 315 & 7896 & 17 & & Linear & none & Palmitic-13C16 \\
\hline & C16:1n7 & Palmitoleic acid & 47.9 & 1198 & 0.76 & & Linear & none & Palmitoleic-13C16 \\
\hline & C18:0 & Stearic acid & 80 & 2000 & 4.02 & & Linear & none & Stearic-d35 \\
\hline & C18:1n7 & cis-Vaccenic acid & 31.6 & 790 & 1.06 & & Linear & none & cis-Vaccenic-13C5 \\
\hline & C18:1n9 & Oleic acid & 240 & 5994 & 14.3 & & Linear & none & Oleic-13C18 \\
\hline & $\mathrm{C} 18: 2 \mathrm{n} 6$ & Linoleic acid & 320 & 8007 & 4.9 & & Linear & none & Linoleic-13C18 \\
\hline & C18:3n3 & alpha-Linolenic acid & 15.9 & 398 & 0.82 & & Linear & none & alpha-Linolenic-d14 \\
\hline & C18:3n6 & gamma-Linolenic acid & 8.18 & 204 & 0.43 & & Linear & none & alpha-Linolenic-d14 \\
\hline & C20:0 & Arachidic acid & 8.05 & 201 & 0.47 & & Linear & none & Arachicic-d39 \\
\hline & C20:1n9 & 11-Eicosenoic acid & NA & NA & NA & NA & NA & NA & NA \\
\hline & $\mathrm{C} 20: 2 \mathrm{n} 6$ & 11,14-Eicosadienoic acid & 2.07 & 51.7 & 0.16 & & Linear & none & Arachicic-d39 \\
\hline & C20:3n6 & homo-gamma-Linolenic acid & 9.96 & 249 & 1 & & Linear & none & Arachidonic-d8 \\
\hline & C20:4n6 & Arachidonic acid & 79.3 & 1983 & 0.36 & & Linear & none & Arachidonic-d8 \\
\hline & C20:5n3 & Eicosapentaenoic acid & 31.4 & 786 & 1.29 & & Linear & none & Eicosapentaenoic-d5 \\
\hline & C22:0 & Docosanoic acid & 8.02 & 200 & 1.77 & & Linear & none & Docosanoic-d43 \\
\hline & C22:1n9 & Docosenoic acid & NA & NA & NA & NA & NA & NA & NA \\
\hline & C22:4n6 & Docosatetraenoic acid & 4.15 & 104 & 0.34 & & Linear & none & Docosahexaenoic-d5 \\
\hline & C22:5n3 & Docosapentaenoic acid & 8.15 & 204 & 0.51 & & Linear & none & Docosahexaenoic-d5 \\
\hline & C22:5n6 & Docosapentaenoic acid & 4.23 & 106 & 0.33 & & Linear & none & Docosahexaenoic-d5 \\
\hline & C22:6n3 & Docosahexaenoic acid & 40 & 999 & 1.96 & & Linear & none & Docosahexaenoic-d5 \\
\hline & C24:0 & Lignoceric acid & 8.05 & 201 & 1.59 & & Linear & none & Lignoceric-d47 \\
\hline & C24:1n9 & Nervonic acid & 8.03 & 201 & 1.38 & & Linear & none & Lignoceric-d47 \\
\hline 10 & C 10:0 & Capric acid & 0.0839 & 4028.7492 & 2.6858 & 26.8583 & Linear & Equal weight $\%$ & Methyl-nonadecanoate \\
\hline & C 12:0 & Lauric acid & 0.0729 & 3501.5101 & 2.3343 & 23.3434 & Linear & Equal weight\% & Methyl-nonadecanoate \\
\hline & C 14:0 & Myristic acid & 0.0645 & 3096.2999 & 2.0642 & 20.6420 & Linear & Equal weight $\%$ & Methyl-nonadecanoate \\
\hline & C 16:0 & Palmitic acid & 0.0578 & 2775.1476 & 1.8501 & 18.5010 & Linear & Equal weight $\%$ & Methyl-nonadecanoate \\
\hline & C $18: 0$ & Stearic Acid & 0.0524 & 2514.3553 & 1.6762 & 16.7624 & Linear & Equal weight $\%$ & Methyl-nonadecanoate \\
\hline & C 20:0 & Arachidic acid & 0.0479 & 2298.3680 & 1.5322 & 15.3225 & Linear & Equal weight $\%$ & Methyl-nonadecanoate \\
\hline & C 22:0 & Docosanoic acid & 0.0441 & 2116.5526 & 1.4110 & 14.1104 & Linear & Equal weight $\%$ & Methyl-nonadecanoate \\
\hline & C 24:0 & Lignoceric acid & 0.0409 & 1961.3940 & 1.3076 & 13.0760 & Linear & Equal weight $\%$ & Methyl-nonadecanoate \\
\hline & C 12:1 & Dodecenoic acid & 0.0736 & 3534.7746 & 2.3565 & 23.5652 & Linear & Equal weight $\%$ & Methyl-nonadecanoate \\
\hline & C 14:1 & Myristoleic acid & 0.0650 & 3122.2823 & 2.0815 & 20.8152 & Linear & Equal weight $\%$ & Methyl-nonadecanoate \\
\hline & C 16:1 & Palmitoleic acid & 0.0583 & 2796.0015 & 1.8640 & 18.6400 & Linear & Equal weight $\%$ & Methyl-nonadecanoate \\
\hline & C 18:1n-7 & cis-Vaccenic acid & 0.0527 & 2531.4619 & 1.6876 & 16.8764 & Linear & Equal weight\% & Methyl-nonadecanoate \\
\hline & C 18:1n-9 & Oleic acid & 0.0527 & 2531.4619 & 1.6876 & 16.8764 & Linear & Equal weight $\%$ & Methyl-nonadecanoate \\
\hline & C $20: 1 n-9$ & 11-Eicosenoic acid & 0.0482 & 2312.6535 & 1.5418 & 15.4177 & Linear & Equal weight $\%$ & Methyl-nonadecanoate \\
\hline & C 22:1n-9 & Docosenoic acid & 0.0443 & 2128.6614 & 1.4191 & 14.1911 & Linear & Equal weight $\%$ & Methyl-nonadecanoate \\
\hline & C 24:1n-9 & Nervonic acid & 0.0411 & 1971.7882 & 1.3145 & 13.1453 & Linear & Equal weight\% & Methyl-nonadecanoate \\
\hline & C 18:2n-6 & Linoleic acid & 0.0531 & 2548.8019 & 1.6992 & 16.9920 & Linear & Equal weight\% & Methyl-nonadecanoate \\
\hline & C 18:3n-6 & gamma-Linolenic acid & 0.0535 & 2566.3820 & 1.7109 & 17.1092 & Linear & Equal weight $\%$ & Methyl-nonadecanoate \\
\hline & C $20: 2 n-6$ & 11,14-Eicosadienoic acid & 0.0485 & 2327.1169 & 1.5514 & 15.5141 & Linear & Equal weight $\%$ & Methyl-nonadecanoate \\
\hline & C 20:3n-6 & hommo-gamma-Linolenic acid & 0.0488 & 2341.7632 & 1.5612 & 15.6118 & Linear & Equal weight $\%$ & Methyl-nonadecanoate \\
\hline & C 20:4n-6 & Arachidonic acid & 0.0491 & 2356.5942 & 1.5711 & 15.7106 & Linear & Equal weight\% & Methyl-nonadecanoate \\
\hline & C 22:2n-6 & Docosadienoic acid & 0.0446 & 2140.9089 & 1.4273 & 14.2727 & Linear & Equal weight\% & Methyl-nonadecanoate \\
\hline & C 22:4n-6 & Docosatetraenoic acid & 0.0451 & 2165.8323 & 1.4439 & 14.4389 & Linear & Equal weight $\%$ & Methyl-nonadecanoate \\
\hline & C 18:3n-3 & alpha-Linolenic acid & 0.0535 & 2566.3820 & 1.7109 & 17.1092 & Linear & Equal weight $\%$ & Methyl-nonadecanoate \\
\hline & C 20:3n-3 & Eicosatrienoic acid & 0.0488 & 2341.7632 & 1.5612 & 15.6118 & Linear & Equal weight $\%$ & Methyl-nonadecanoate \\
\hline & C 20:5n-3 & Eicosapentaenoic acid & 0.0494 & 2371.6150 & 1.5811 & 15.8108 & Linear & Equal weight $\%$ & Methyl-nonadecanoate \\
\hline & C 22:5n-3 & Docosapentaenoic acid, n3 & 0.0454 & 2178.5132 & 1.4523 & 14.5234 & Linear & Equal weight $\%$ & Methyl-nonadecanoate \\
\hline & C 22:6n-3 & Docosahexaenoic acid & 0.0457 & 2191.3428 & 1.4609 & 14.6090 & Linear & Equal weight $\%$ & Methyl-nonadecanoate \\
\hline
\end{tabular}


Table A-3 (cont) Calibration information

\begin{tabular}{|c|c|c|c|c|c|c|c|c|c|}
\hline \multicolumn{10}{|c|}{ Table A-3 (cont) Calibration information } \\
\hline Lab & $\begin{array}{l}\text { Fatty Acid } \\
\text { Code }\end{array}$ & Fatty Acid Name & $\begin{array}{c}\text { Lowest std } \\
(\mu \mathrm{mol} / \mathrm{L})\end{array}$ & $\begin{array}{c}\text { Highest std } \\
(\mu \mathrm{mol} / \mathrm{L})\end{array}$ & $\begin{array}{c}\mathrm{LOD} \\
(\mu \mathrm{mol} / \mathrm{L})\end{array}$ & $\begin{array}{c}\mathrm{LOQ} \\
(\mu \mathrm{mol} / \mathrm{L})\end{array}$ & Curve Type & Weighting & IS used for quantitation \\
\hline 11 & No information & & & & & & & & \\
\hline \multirow[t]{36}{*}{12} & C14:0 & Myristic Acid & 0.0495 & 165 & & & quadratic & $1 / \mathrm{x}$ & Glyceryl Tri(hexadecanoate-d31) \\
\hline & C14:1n5 & Myristoleic Acid & 0.0083 & 27.7 & & & quadratic & $1 / x$ & Glyceryl Tri(hexadecanoate-d31) \\
\hline & C16:0 & Palmitic Acid & 0.0592 & 197 & & & quadratic & $1 / x$ & Glyceryl Tri(hexadecanoate-d31) \\
\hline & C16:1n7 & Palmitoleic Acid & 0.0075 & 24.8 & & & quadratic & $1 / x$ & Glyceryl Tri(hexadecanoate-d31) \\
\hline & C18:0 & Stearic Acid & 0.0402 & 134 & & & quadratic & $1 / x$ & Glyceryl Tri(hexadecanoate-d31) \\
\hline & C18:1n7 & Vaccenic Acid & 0.0030 & 9.8 & & & quadratic & $1 / x$ & Glyceryl Tri(hexadecanoate-d31) \\
\hline & C18:1n9 & Oleic Acid & 0.0270 & 89.9 & & & quadratic & $1 / x$ & Glyceryl Tri(hexadecanoate-d31) \\
\hline & C18:2n6 & Linoleic Acid & 0.0204 & 67.9 & & & quadratic & $1 / x$ & Glyceryl Tri(hexadecanoate-d31) \\
\hline & C18:3n3 & Alpha Linolenic Acid & 0.0109 & 36.2 & & & quadratic & $1 / x$ & Glyceryl Tri(hexadecanoate-d31) \\
\hline & C18:3n6 & Gamma Linolenic Acid & 0.0040 & 13.4 & & & quadratic & $1 / x$ & Glyceryl Tri(hexadecanoate-d31) \\
\hline & C20:0 & Arachidic Acid & 0.0061 & 20.4 & & & quadratic & $1 / x$ & Glyceryl Tri(hexadecanoate-d31) \\
\hline & C20:1n9 & 11-Eicosenoic Acid & 0.0123 & 41.1 & & & quadratic & $1 / x$ & Glyceryl Tri(hexadecanoate-d31) \\
\hline & C20:2n6 & 11-14-Eicosadienoic Acid & 0.0070 & 23.4 & & & quadratic & $1 / x$ & Glyceryl Tri(hexadecanoate-d31) \\
\hline & C20:3n6 & Homogamma Linolenic Acid & 0.0036 & 12.0 & & & quadratic & $1 / x$ & Glyceryl Tri(hexadecanoate-d31) \\
\hline & C20:4n6 & Arachidonic Acid & 0.0344 & 114.8 & & & quadratic & $1 / x$ & Glyceryl Tri(hexadecanoate-d31) \\
\hline & C20:5n3 & Eicosapentaenoic Acid & 0.0051 & 17.0 & & & quadratic & $1 / x$ & Glyceryl Tri(hexadecanoate-d31) \\
\hline & C22:0 & Behenic Acid & 0.0056 & 18.8 & & & quadratic & $1 / x$ & Glyceryl Tri(hexadecanoate-d31) \\
\hline & C22:1n9 & Erucic Acid & 0.0075 & 25.1 & & & quadratic & $1 / x$ & Glyceryl Tri(hexadecanoate-d31) \\
\hline & C22:4n6 & Docosic Acidtraenoic Acid & 0.0081 & 27.0 & & & quadratic & $1 / x$ & Glyceryl Tri(hexadecanoate-d31) \\
\hline & C22:5n3 & Docosapentaenoic Acid & 0.0068 & 22.7 & & & quadratic & $1 / x$ & Glyceryl Tri(hexadecanoate-d31) \\
\hline & C22:5n6 & Docosapentaenoic Acid N6 & 0.0078 & 26.0 & & & quadratic & $1 / x$ & Glyceryl Tri(hexadecanoate-d31) \\
\hline & C22:6n3 & Docosahexaenoic Acid (DHA) & 0.0188 & 62.7 & & & quadratic & $1 / x$ & Glyceryl Tri(hexadecanoate-d31) \\
\hline & C24:0 & Ignoceric Acid & 0.0052 & 17.4 & & & quadratic & $1 / x$ & Glyceryl Tri(hexadecanoate-d31) \\
\hline & C24:1n9 & Nervonic Acid & 0.0084 & 27.9 & & & quadratic & $1 / x$ & Glyceryl Tri(hexadecanoate-d31) \\
\hline & C12:0 & Lauric Acid & 0.0373 & 124.4 & & & quadratic & $1 / x$ & Glyceryl Tri(hexadecanoate-d31) \\
\hline & C15:0 & Pentadecanoic Acid & 0.0078 & 26.0 & & & quadratic & $1 / x$ & Glyceryl Tri(hexadecanoate-d31) \\
\hline & C16:1n7t & 9t-Palmitelaidic Acid (Omega 7) & 0.0084 & 27.9 & & & quadratic & $1 / x$ & Glyceryl Tri(hexadecanoate-d31) \\
\hline & C17:0 & Heptadecanoic Acid & 0.0070 & 23.4 & & & quadratic & $1 / x$ & Glyceryl Tri(hexadecanoate-d31) \\
\hline & C17:1n9 & 10-Heptadecenoic Acid & 0.0071 & 23.6 & & & quadratic & $1 / x$ & Glyceryl Tri(hexadecanoate-d31) \\
\hline & 9c,11t-CLA & 9c,11t-Conjugated Linoleic Acid & 0.0073 & 24.5 & & & quadratic & $1 / x$ & Glyceryl Tri(hexadecanoate-d31) \\
\hline & C18:4n3 & Stearidonic Acid & 0.0012 & 4.1 & & & quadratic & $1 / x$ & Glyceryl Tri(hexadecanoate-d31) \\
\hline & C19:0 & Nonadecanoic Acid & 0.0064 & 21.3 & & & quadratic & $1 / x$ & Glyceryl Tri(hexadecanoate-d31) \\
\hline & C20:3n3 & 11-14-17 Eicosatrienoic Acid & 0.0055 & 18.3 & & & quadratic & $1 / x$ & Glyceryl Tri(hexadecanoate-d31) \\
\hline & C20:4n3 & Arachidonic Acid (Omega 3) & 0.0030 & 10.0 & & & quadratic & $1 / x$ & Glyceryl Tri(hexadecanoate-d31) \\
\hline & C21:0 & Heneicosanoic Acid & 0.0031 & 10.3 & & & quadratic & $1 / x$ & Glyceryl Tri(hexadecanoate-d31) \\
\hline & C22:2n6 & 13-16-Docosadienoic Acid & 0.0070 & 23.3 & & & quadratic & $1 / \mathrm{x}$ & Glyceryl Tri(hexadecanoate-d31) \\
\hline 13 & No information & & & & & & & & \\
\hline
\end{tabular}

13 No information 
Table A-4 Additional notes provided by laboratories

\begin{tabular}{|c|l|}
\hline Lab \# & Note: \\
\hline 1 & atrans-vaccenic acid coelutes with cis-vaccenic acid \\
\hline & ND = not determined \\
\hline 2 & C20:1n9 and C20:2n6 were not quantitated \\
\hline 11 & Method was not quantitative so data were withdrawn. \\
\hline 12 (total fatty acid data) & C22:1n9 (Docosenoic acid) was used as a surrogate. \\
\hline 12 (free fatty acid data) & C22:1n9 (Docosenoic acid) was used as a surrogate. \\
\hline
\end{tabular}




\section{Appendix B.}

Charts of interlaboratory data received for the individual fatty acids in each material (in terms of $\mu \mathrm{M}$ ) with the median identified for the three unknown samples and the certified or reference value with associated confidence interval identified for SRM 1950.

The charts are presented in the order of an individual fatty acid in each of the four materials (unknown 004, unknown 005, unknown 006, and SRM 1950) with the fatty acids in the order presented in Table 1 . If fewer than four laboratories reported numerical data for a fatty acid, no chart is presented.

The error bars for each data point represent the laboratory's standard deviation from their analysis of three subsamples. 


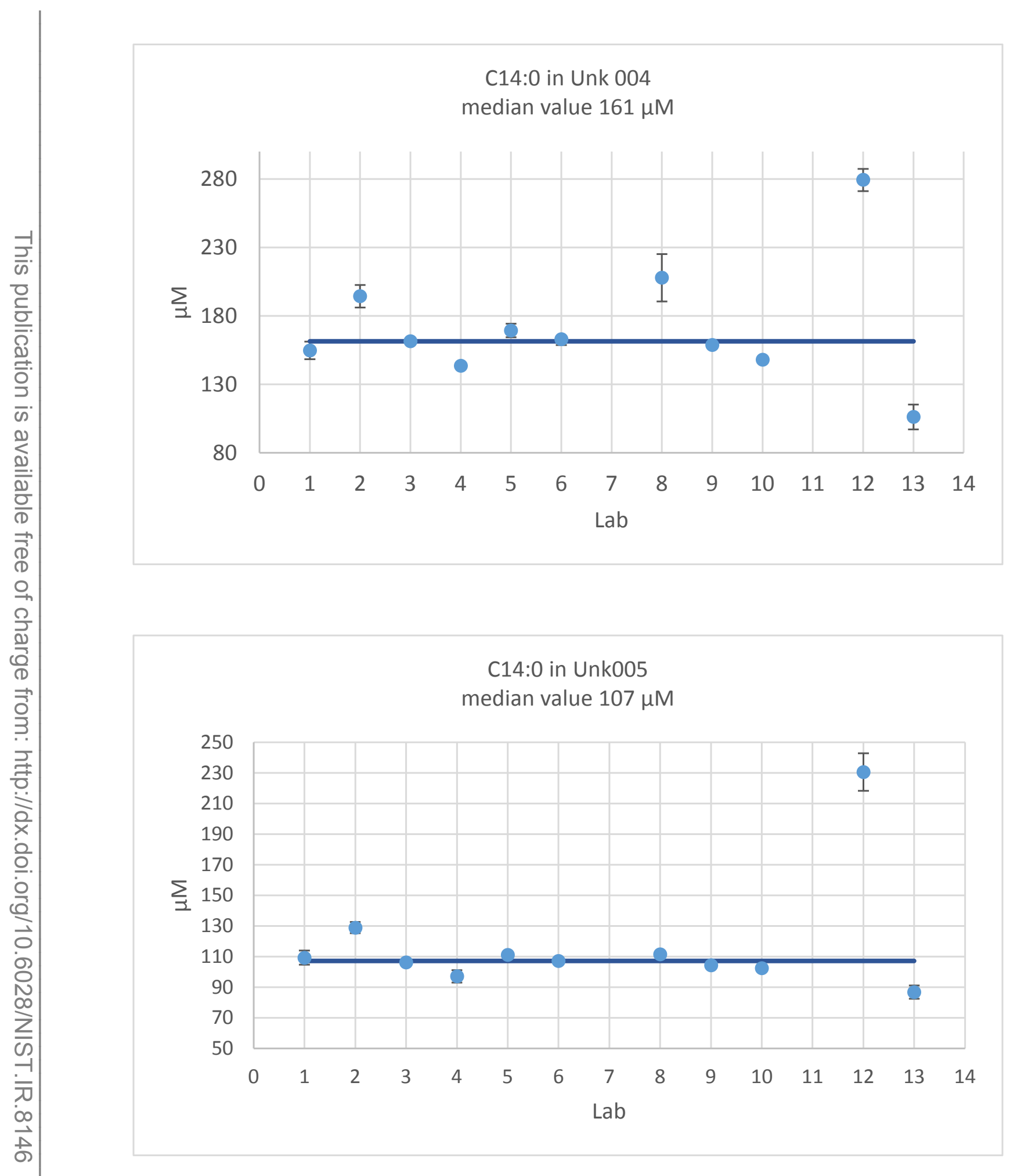



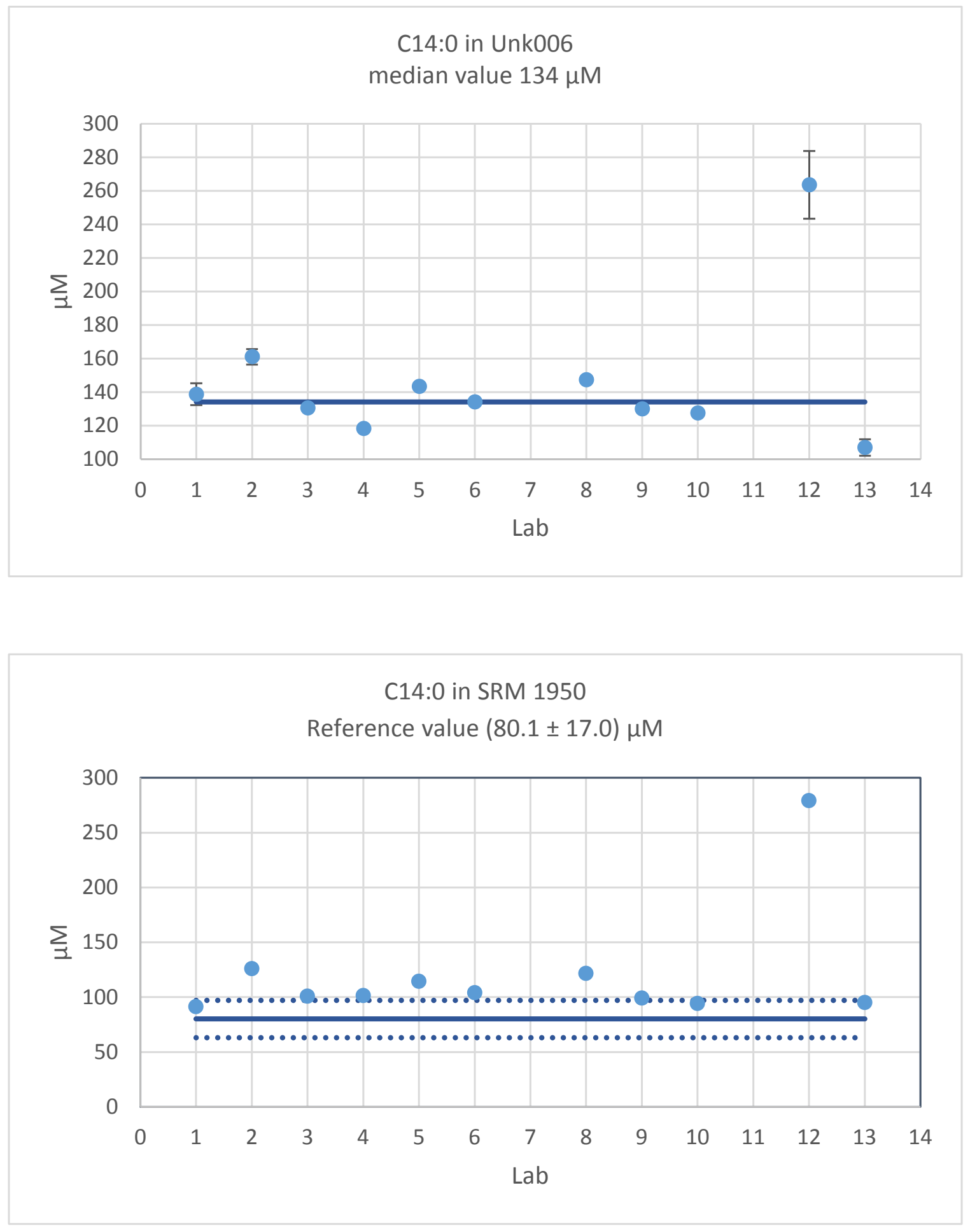


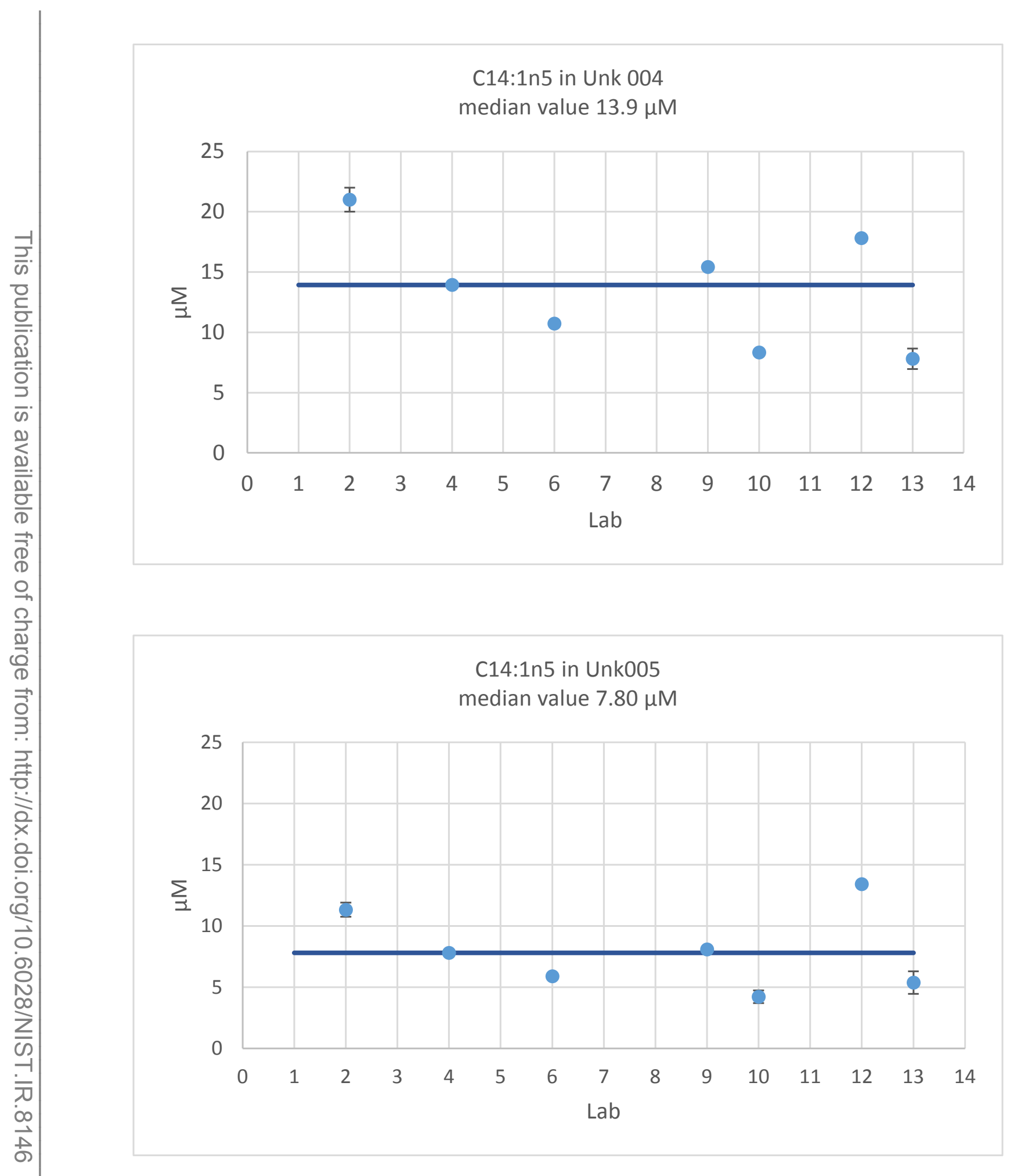




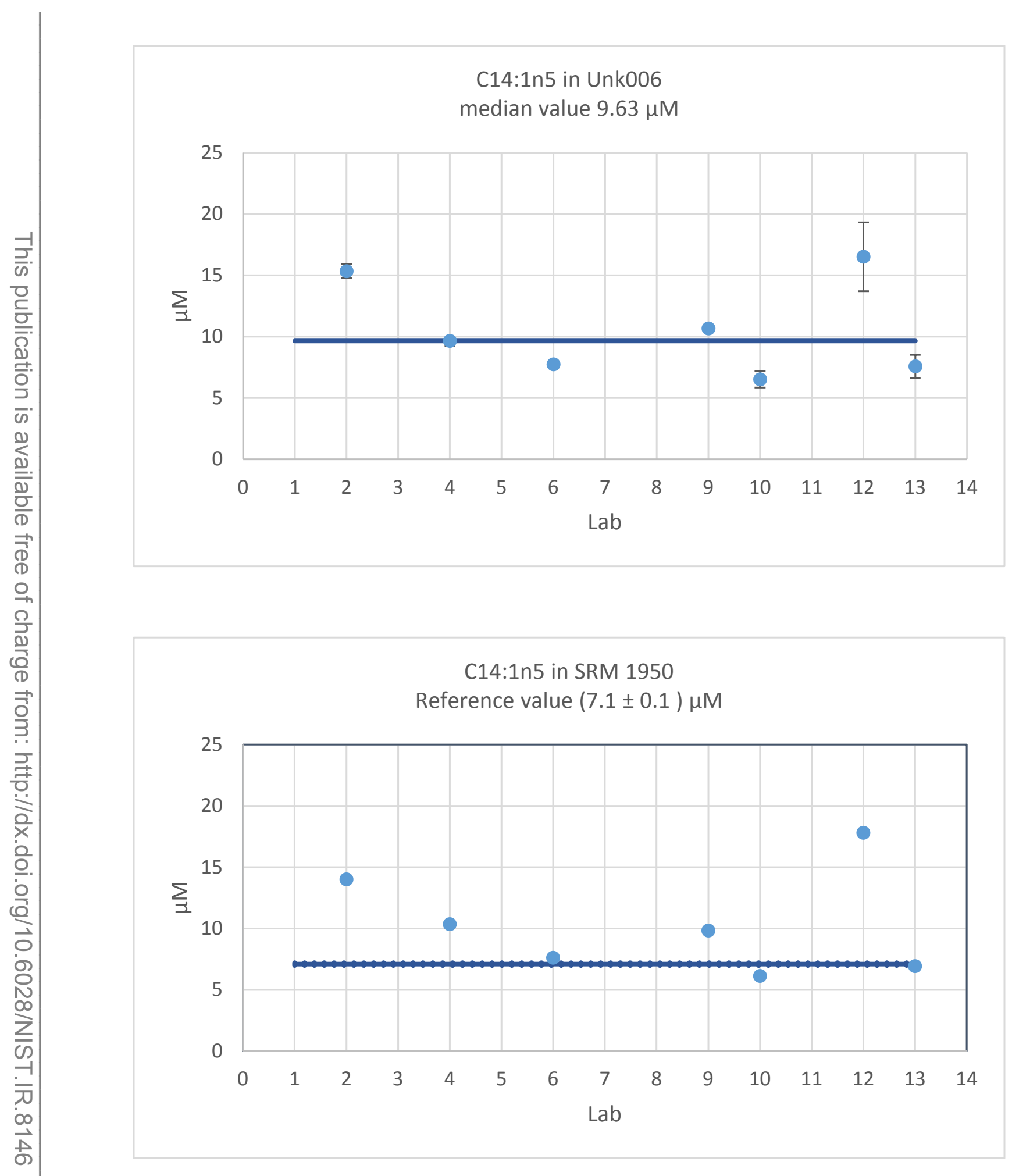




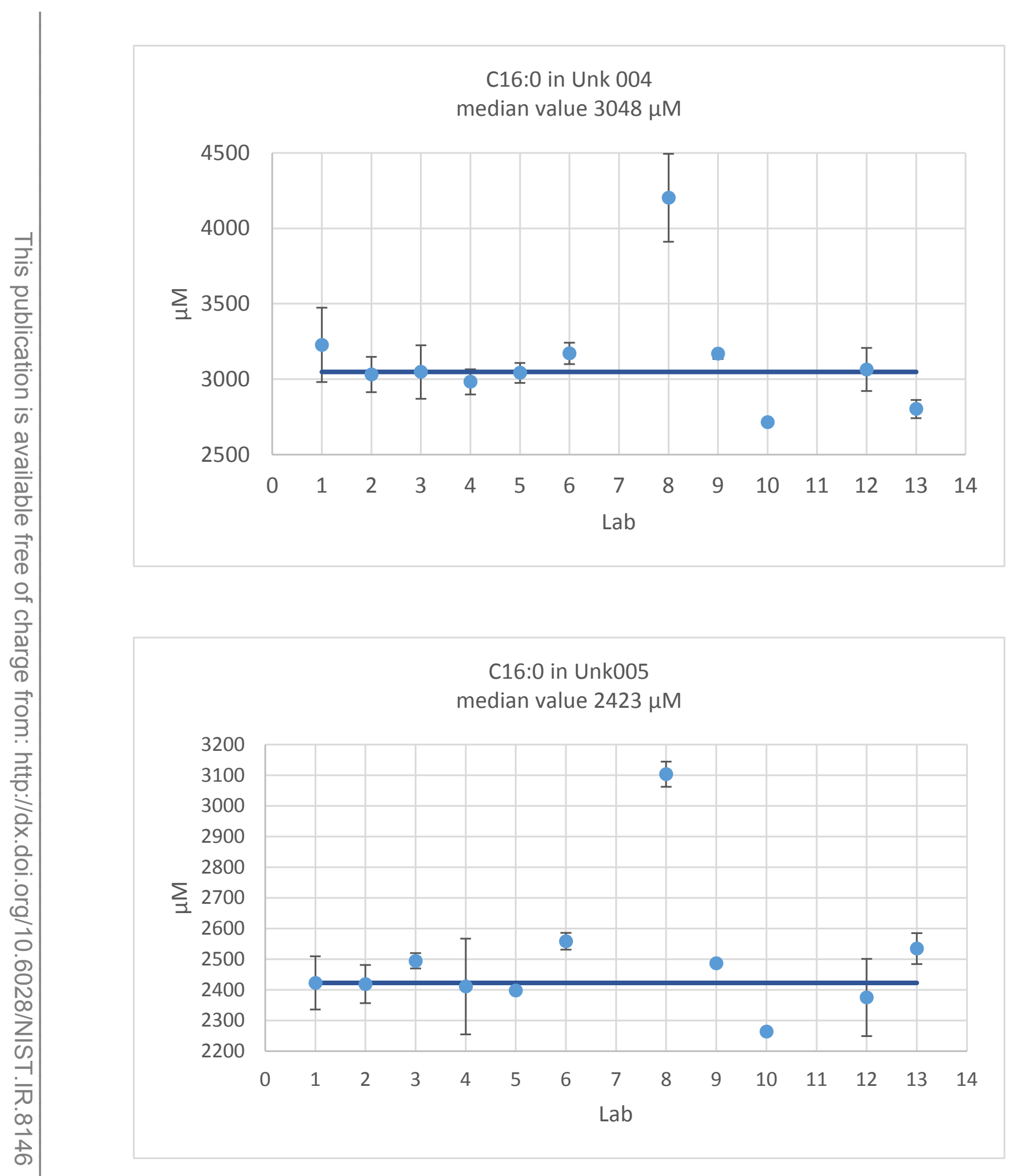




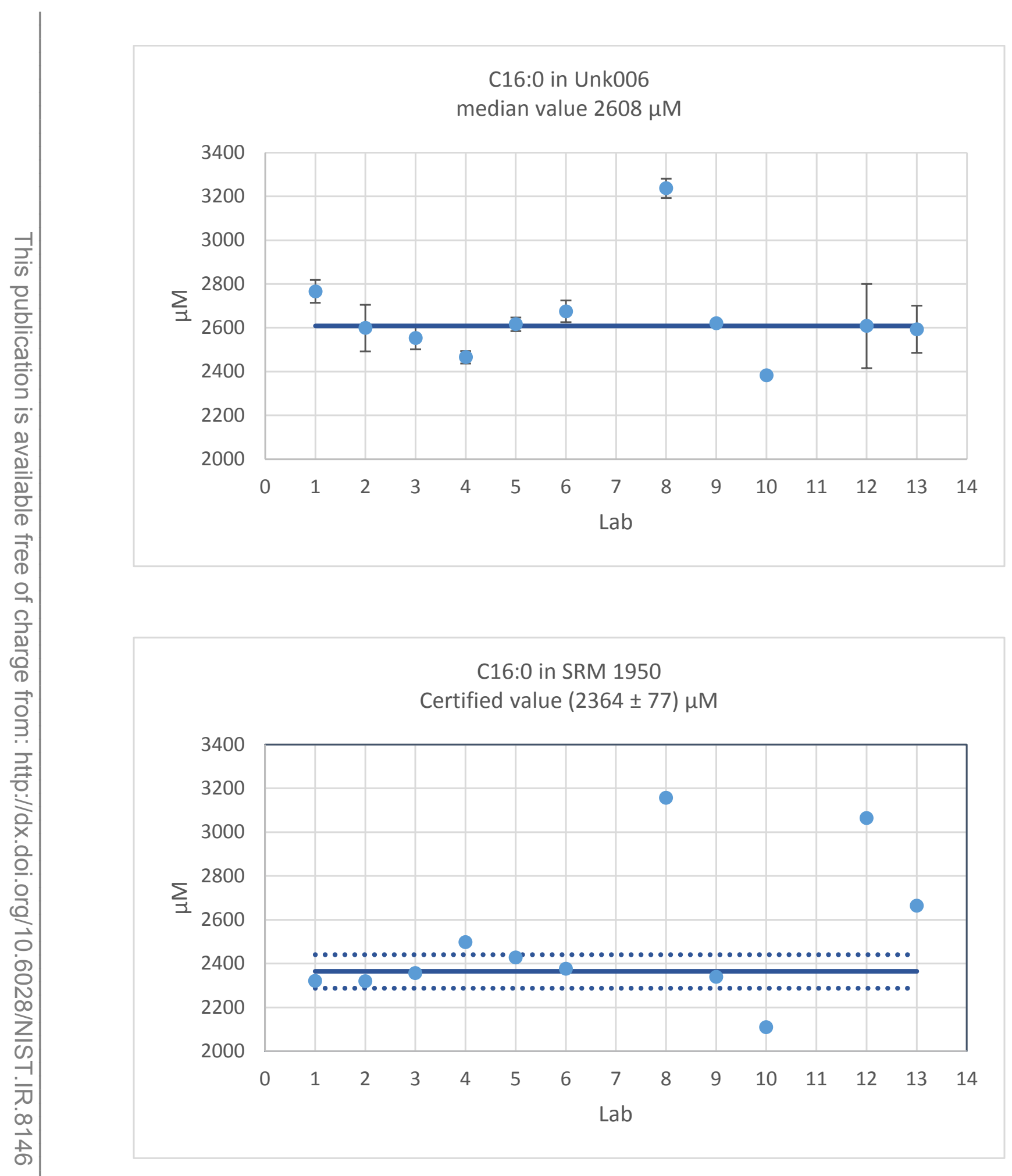



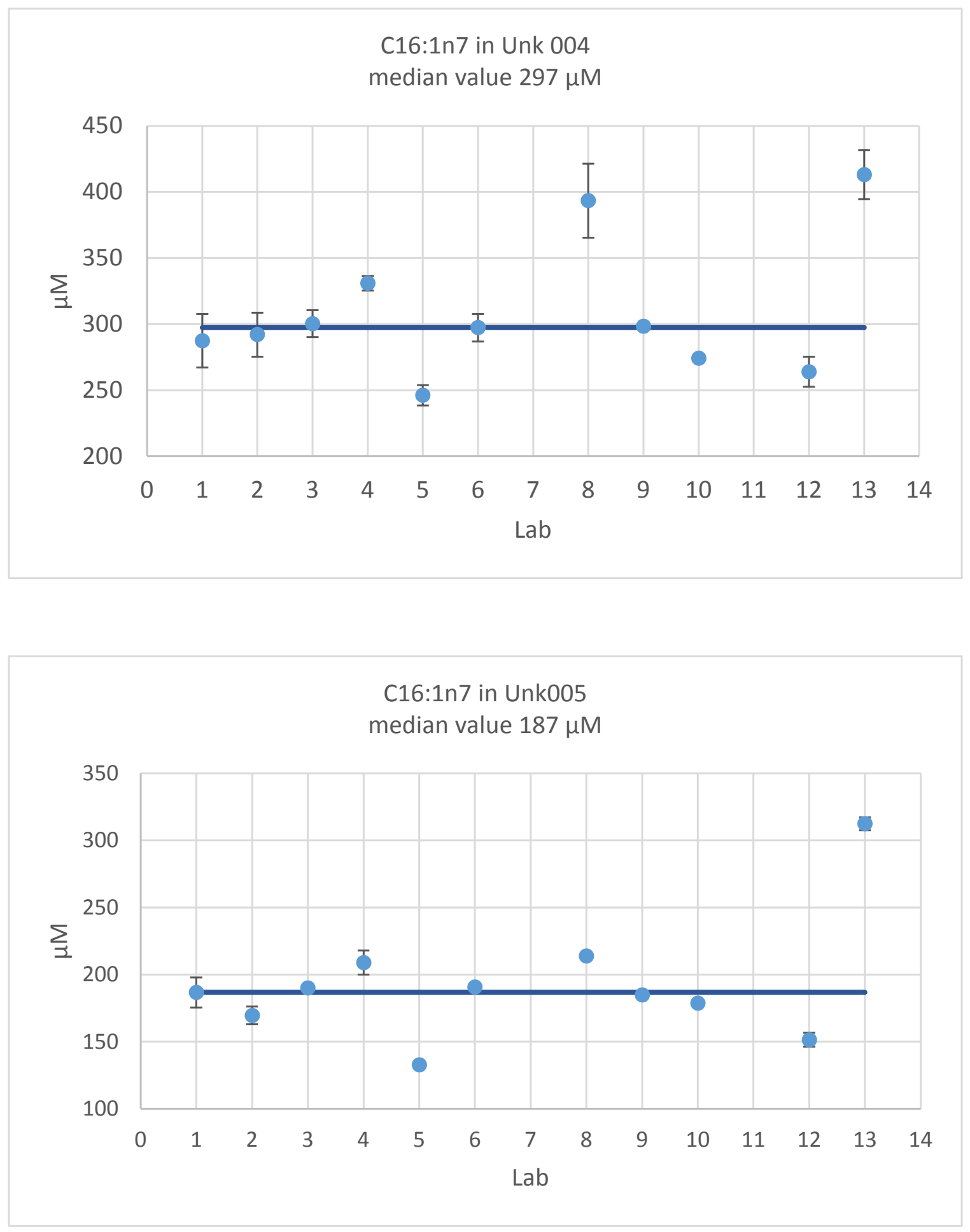


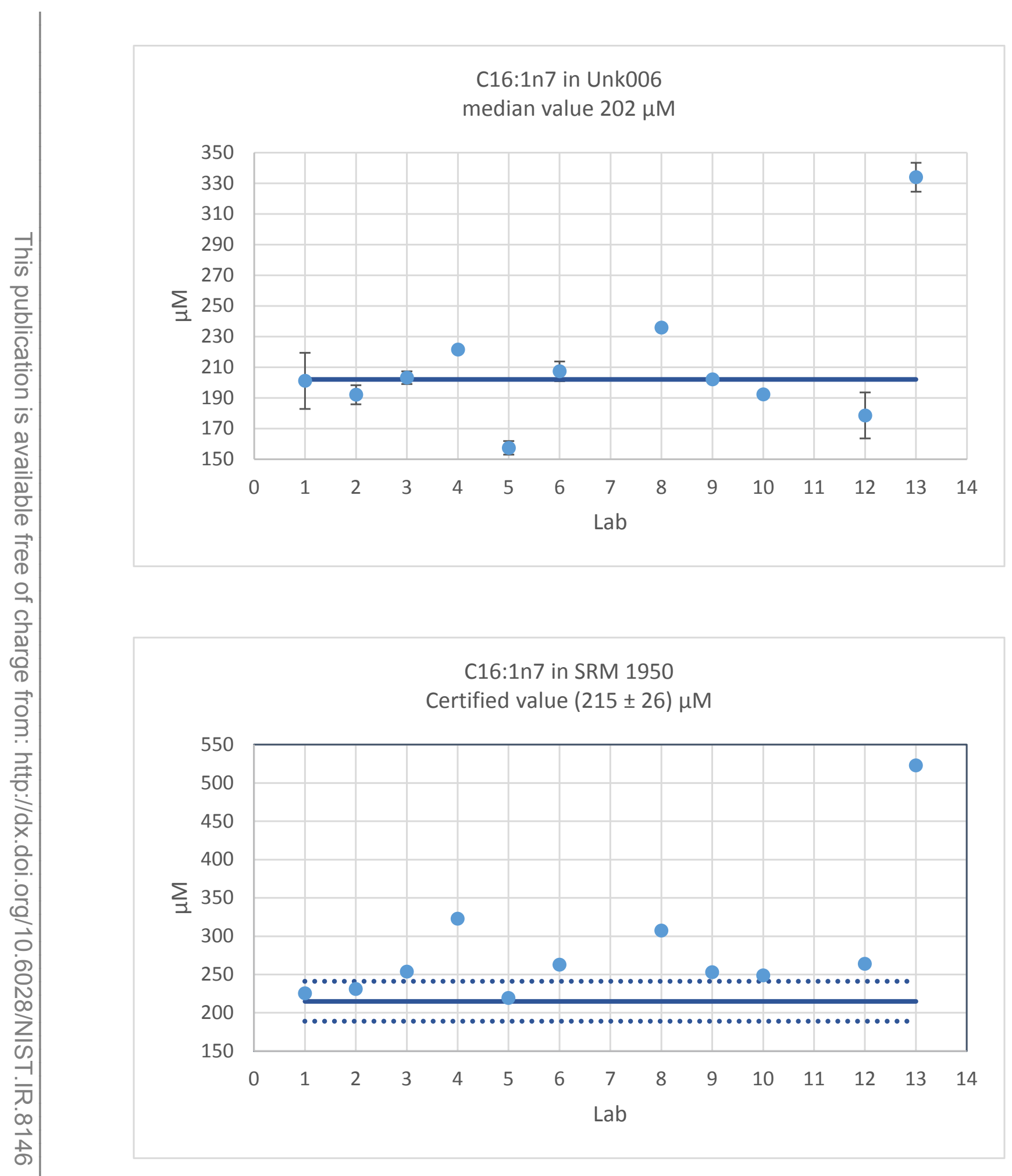




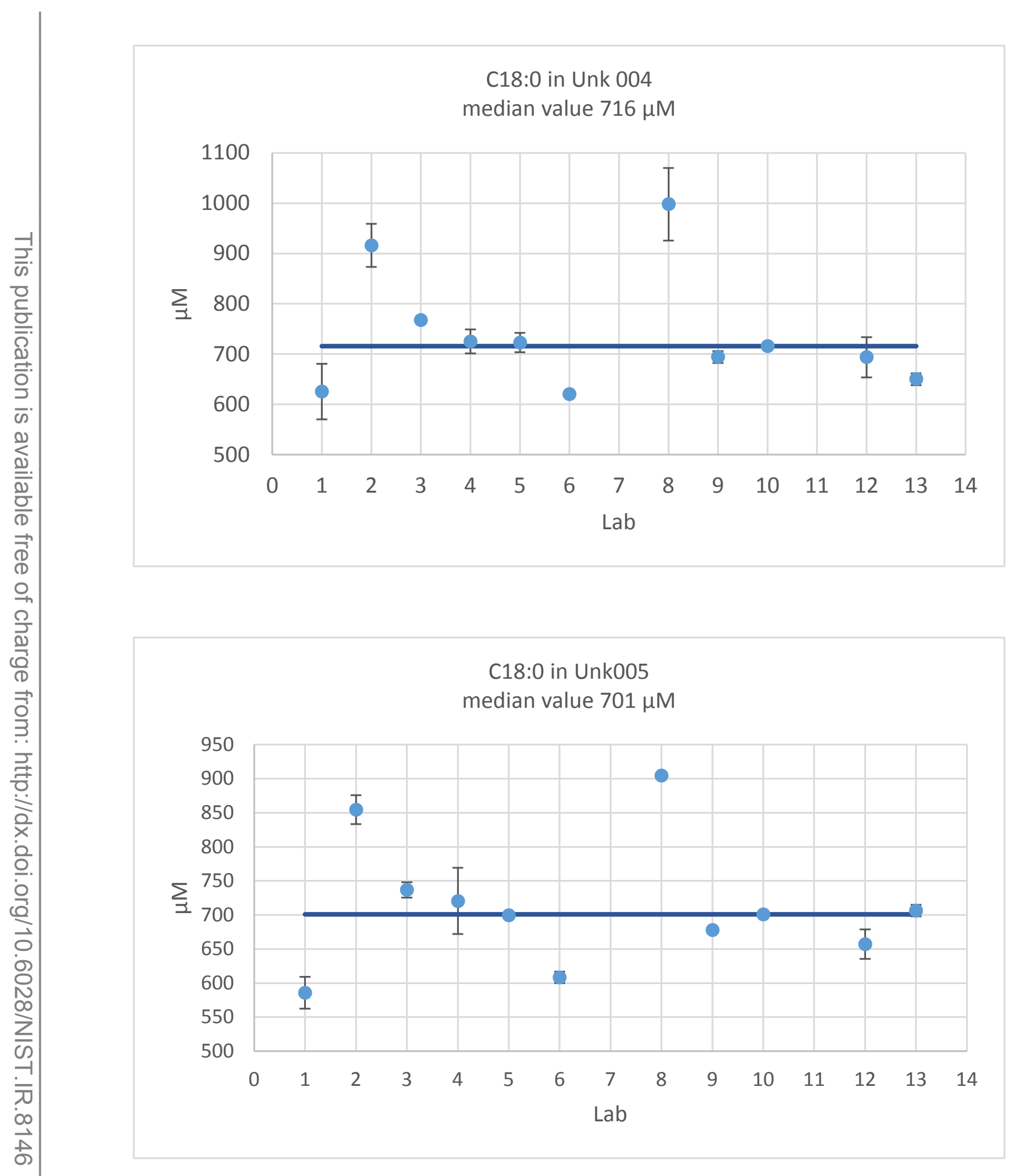




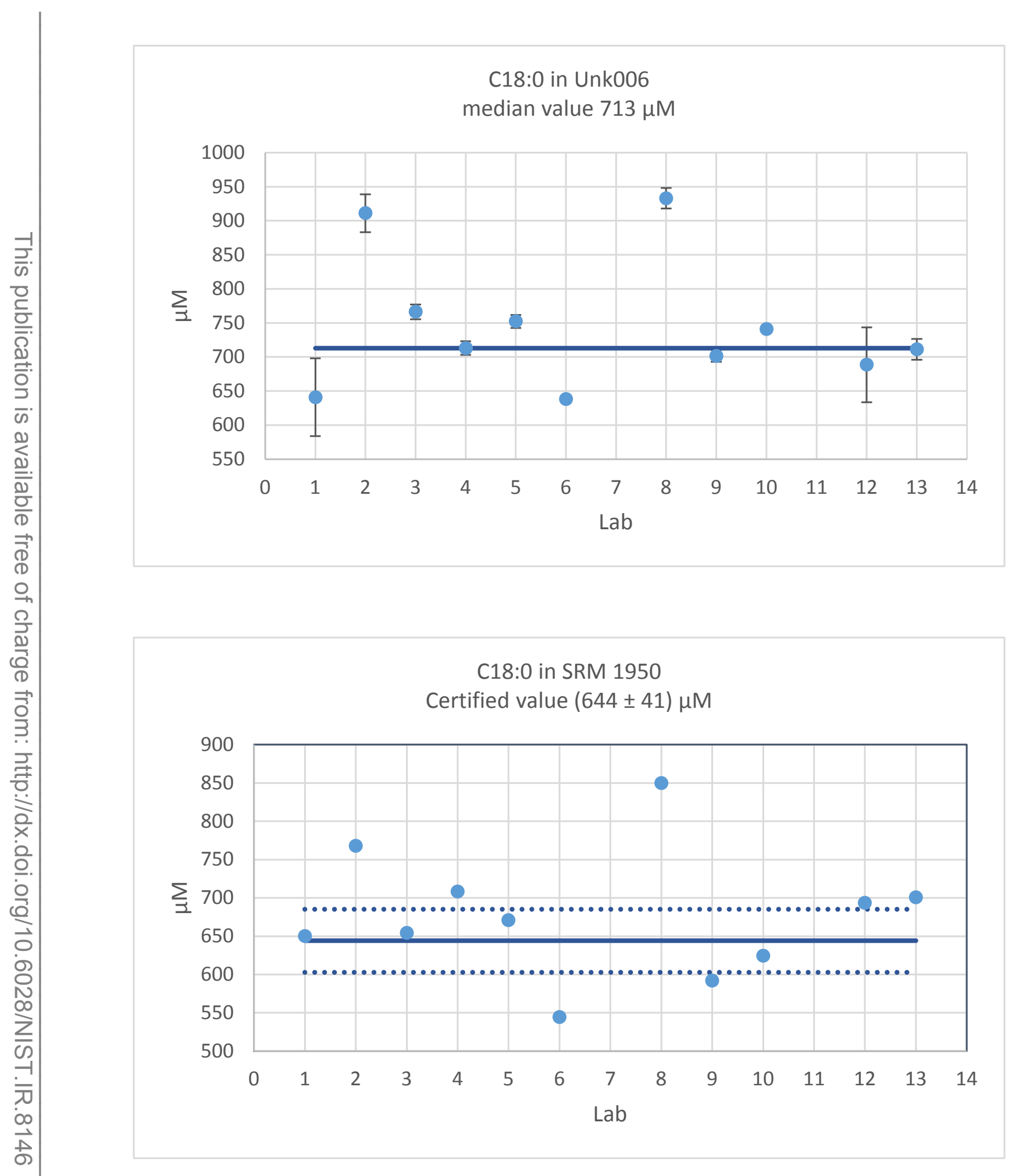



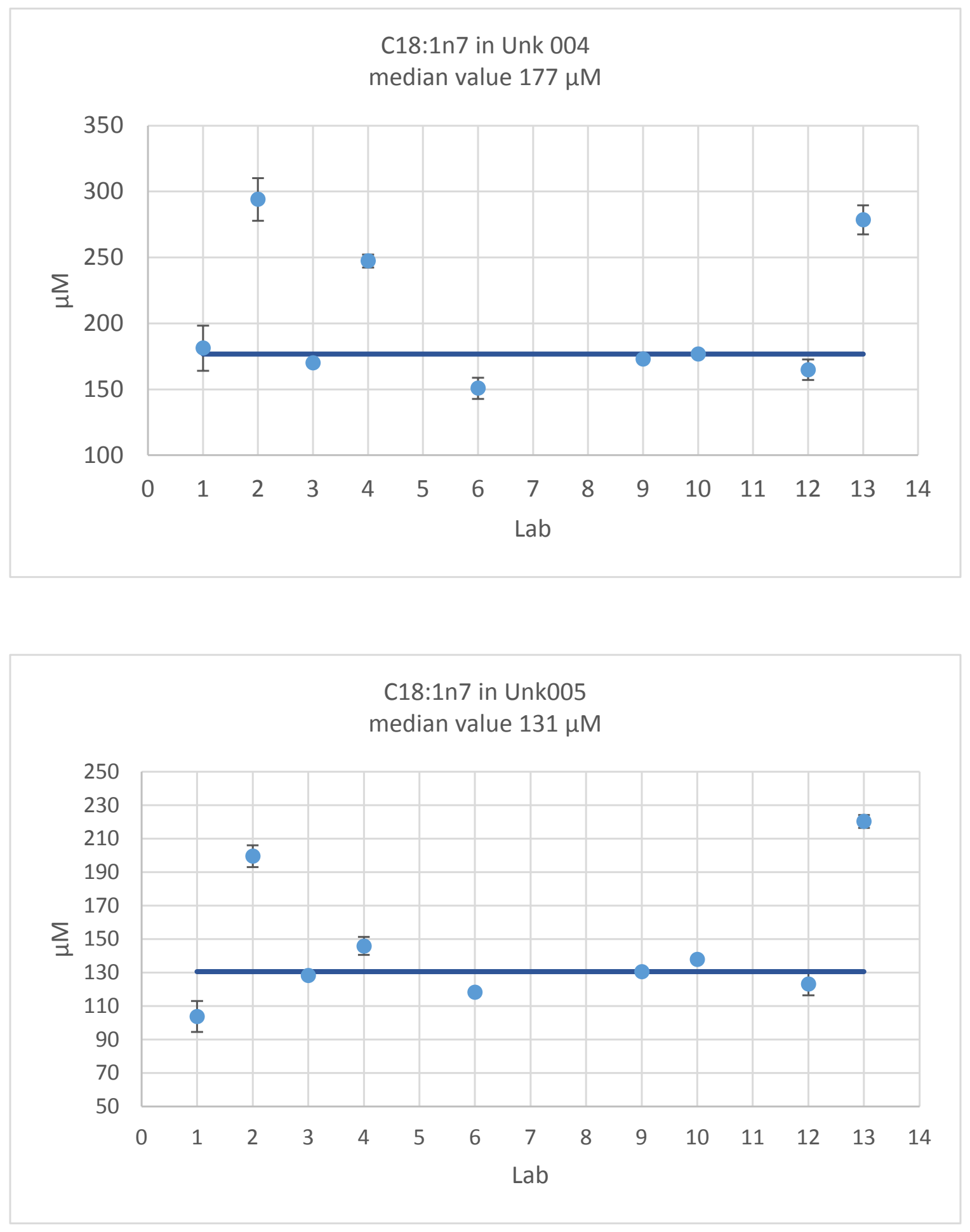


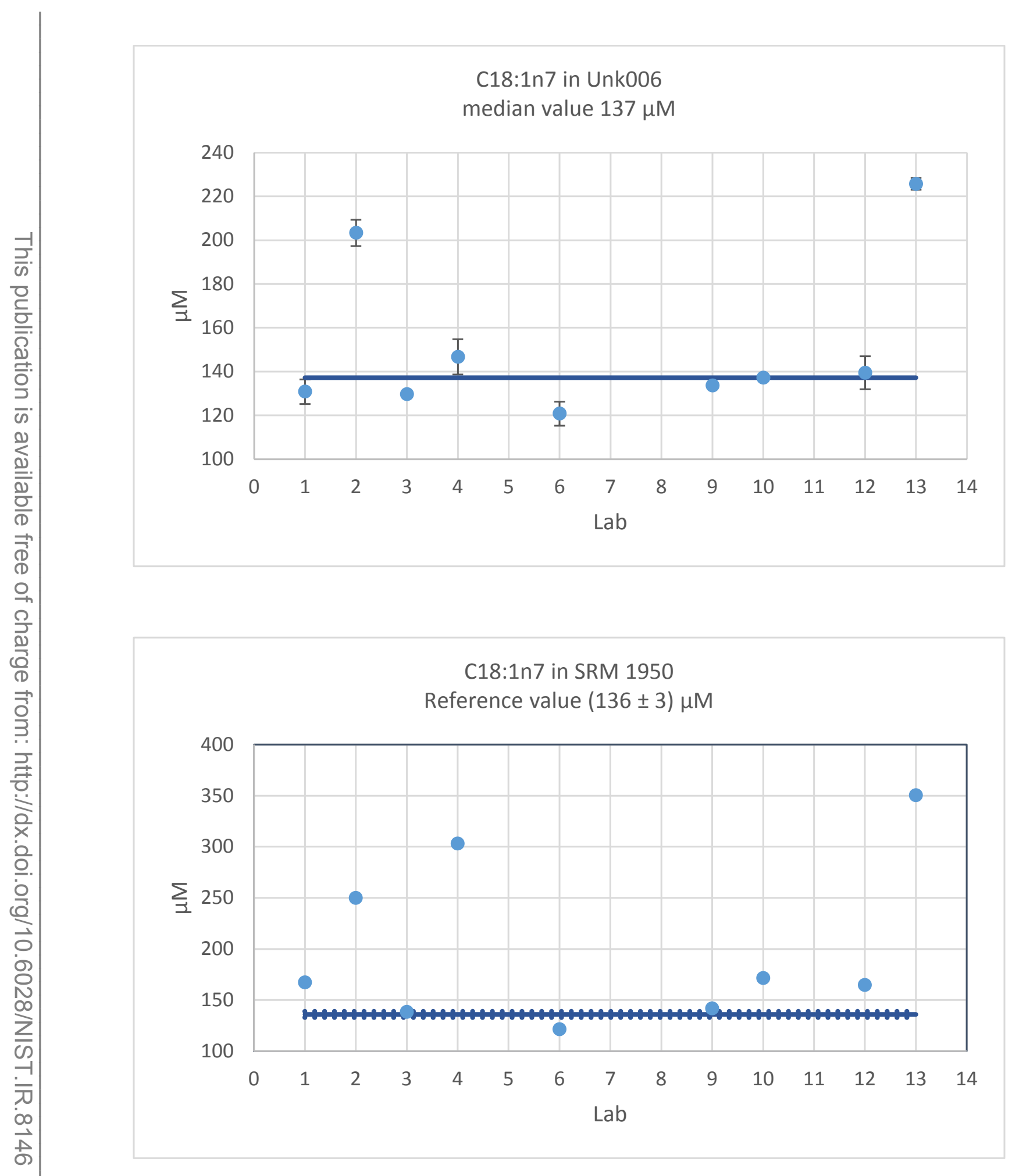



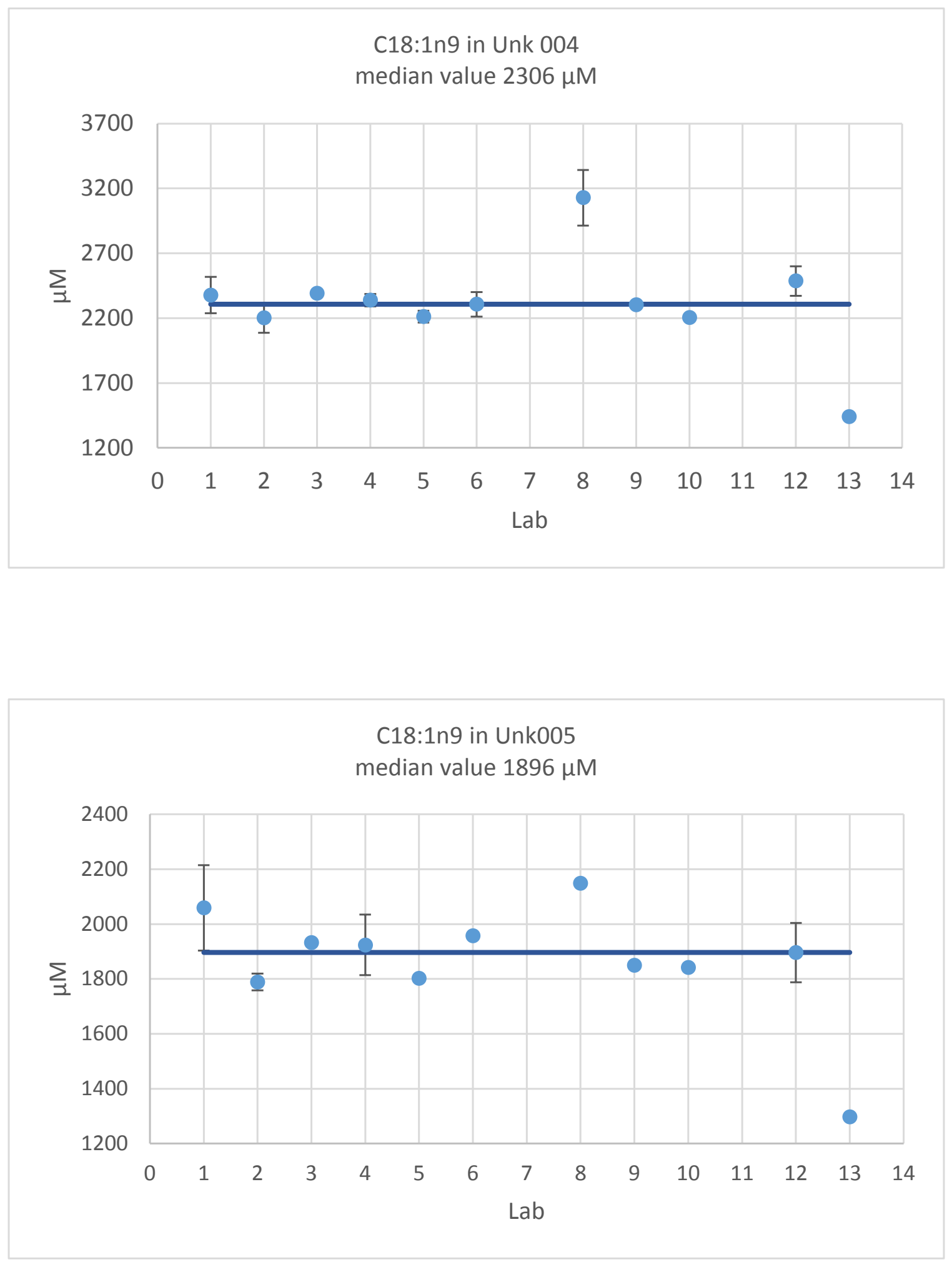


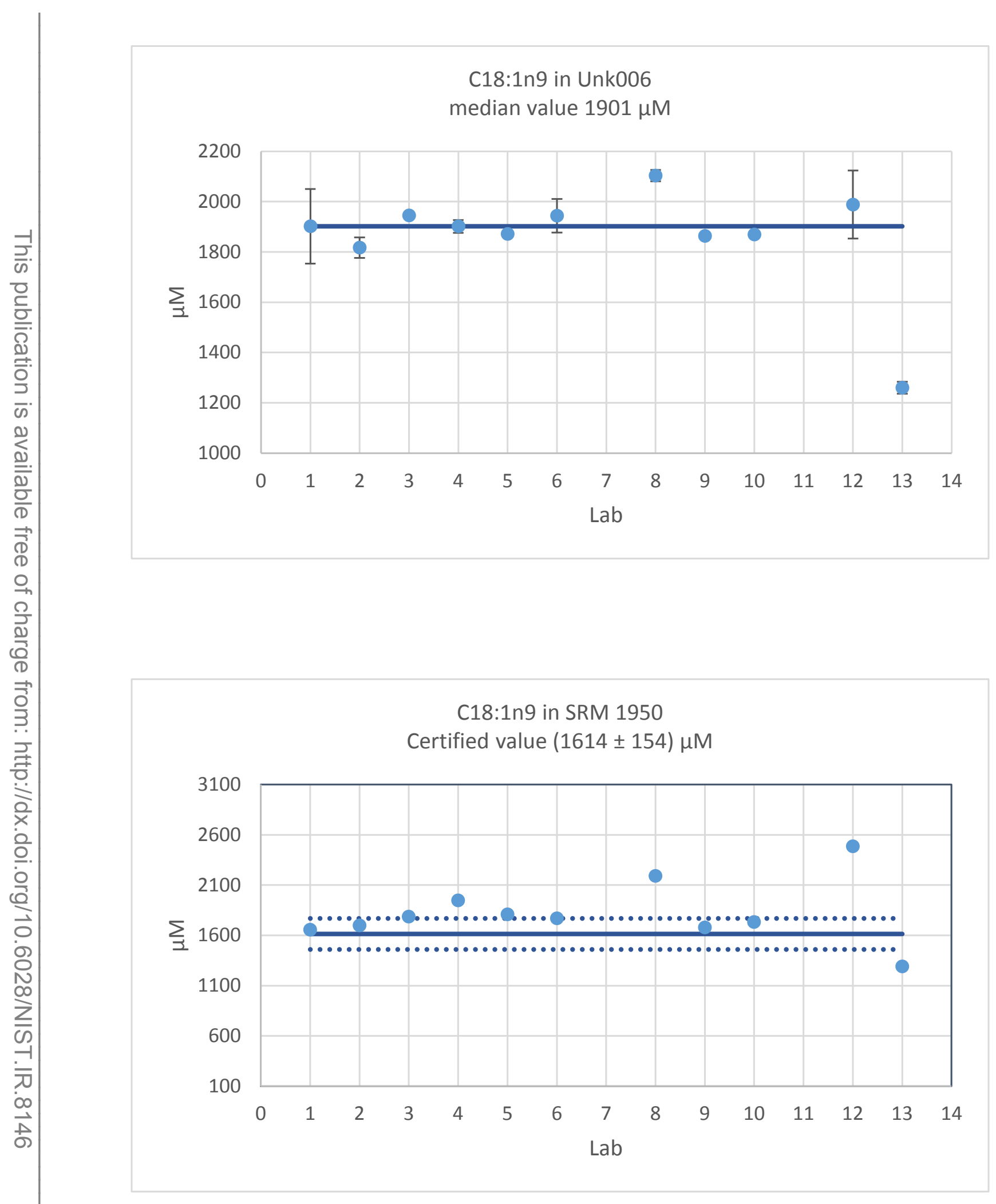



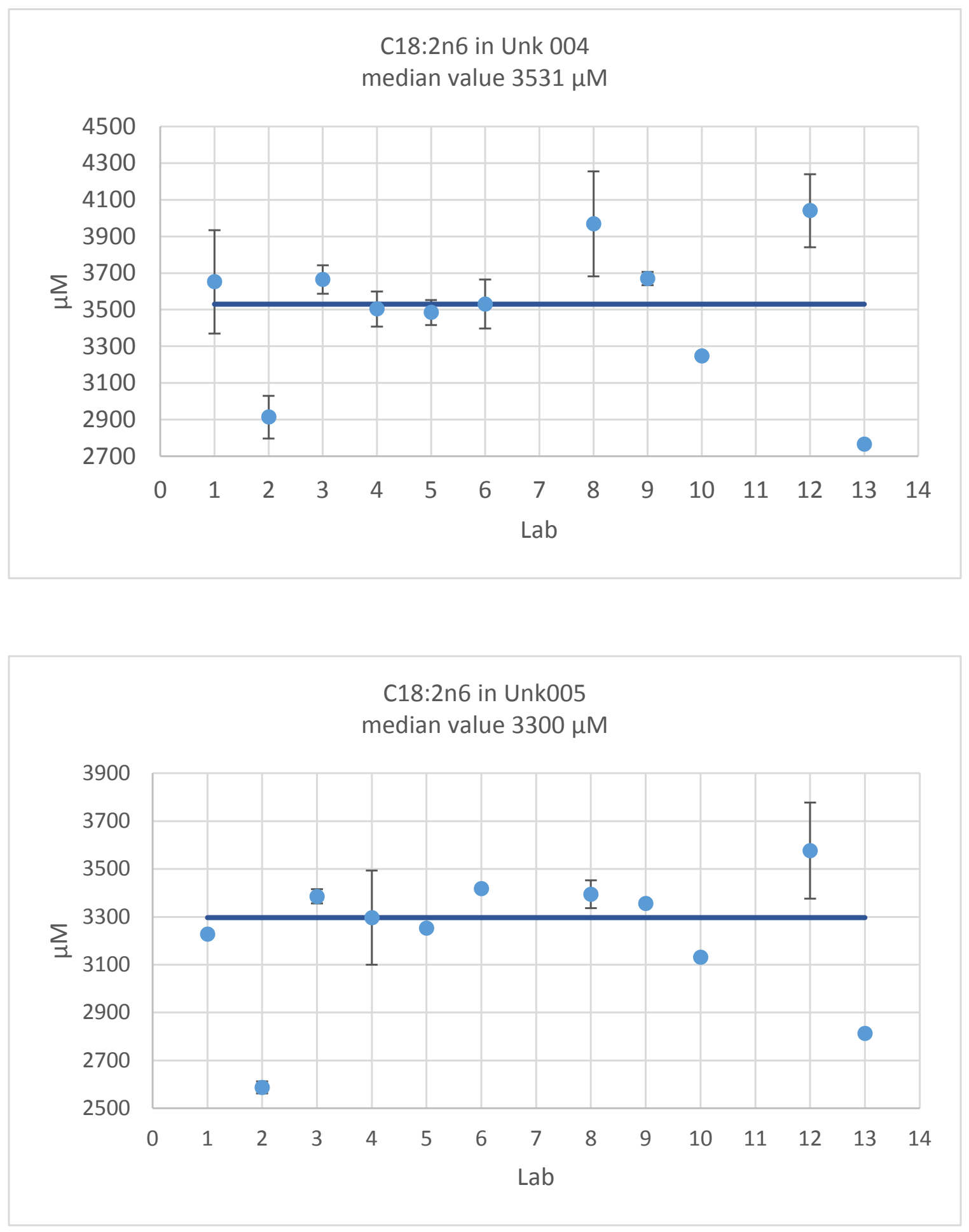


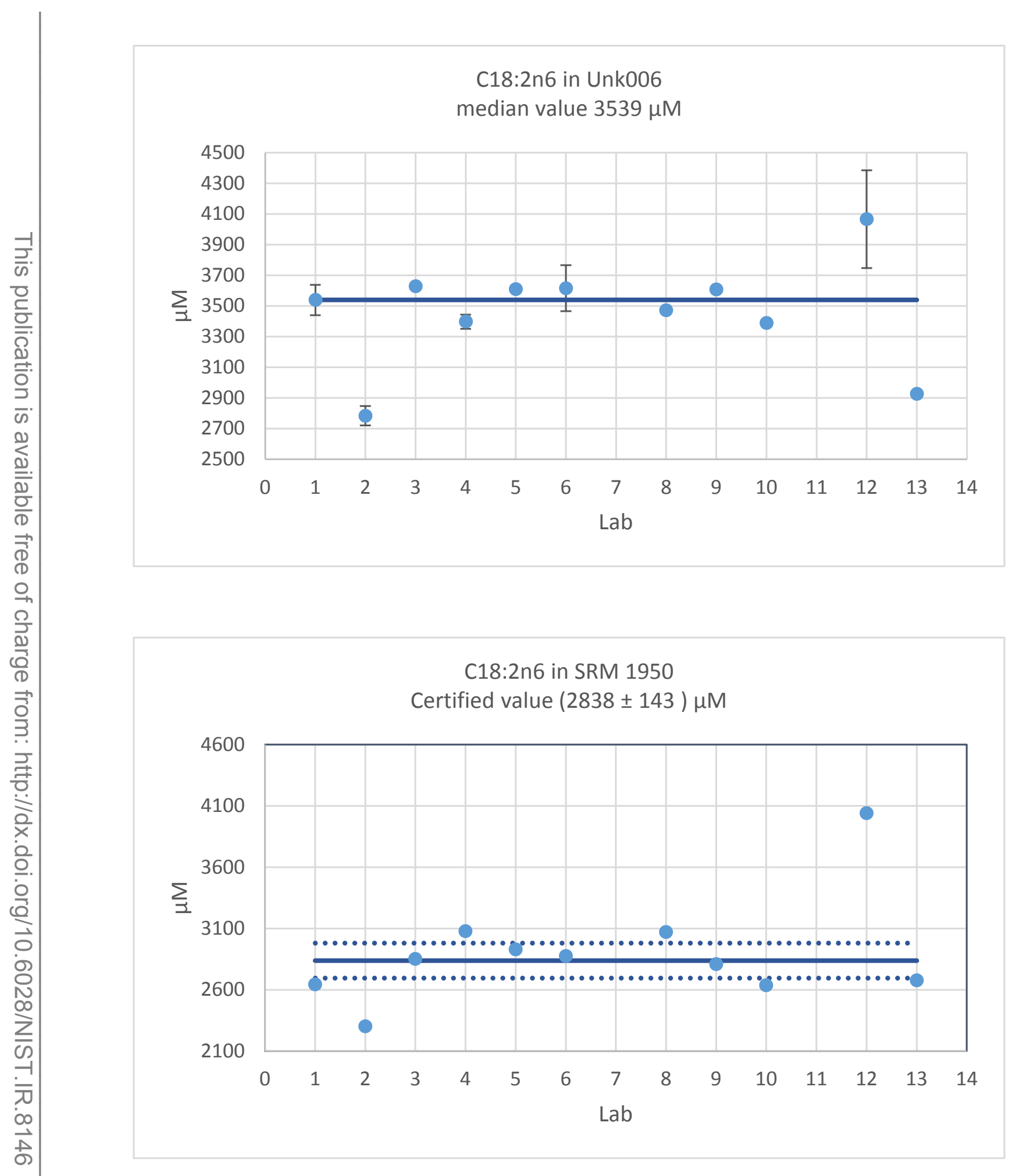




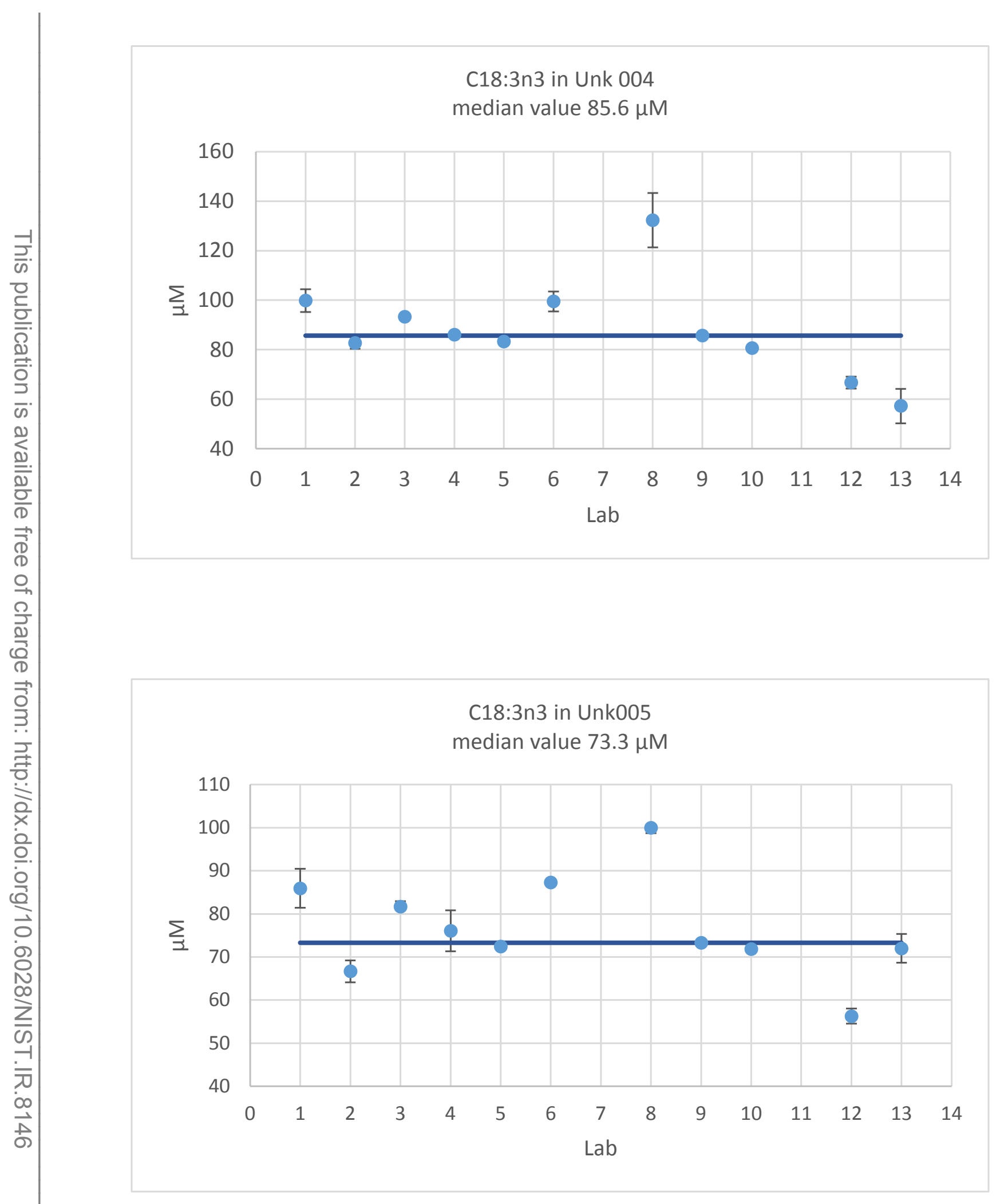




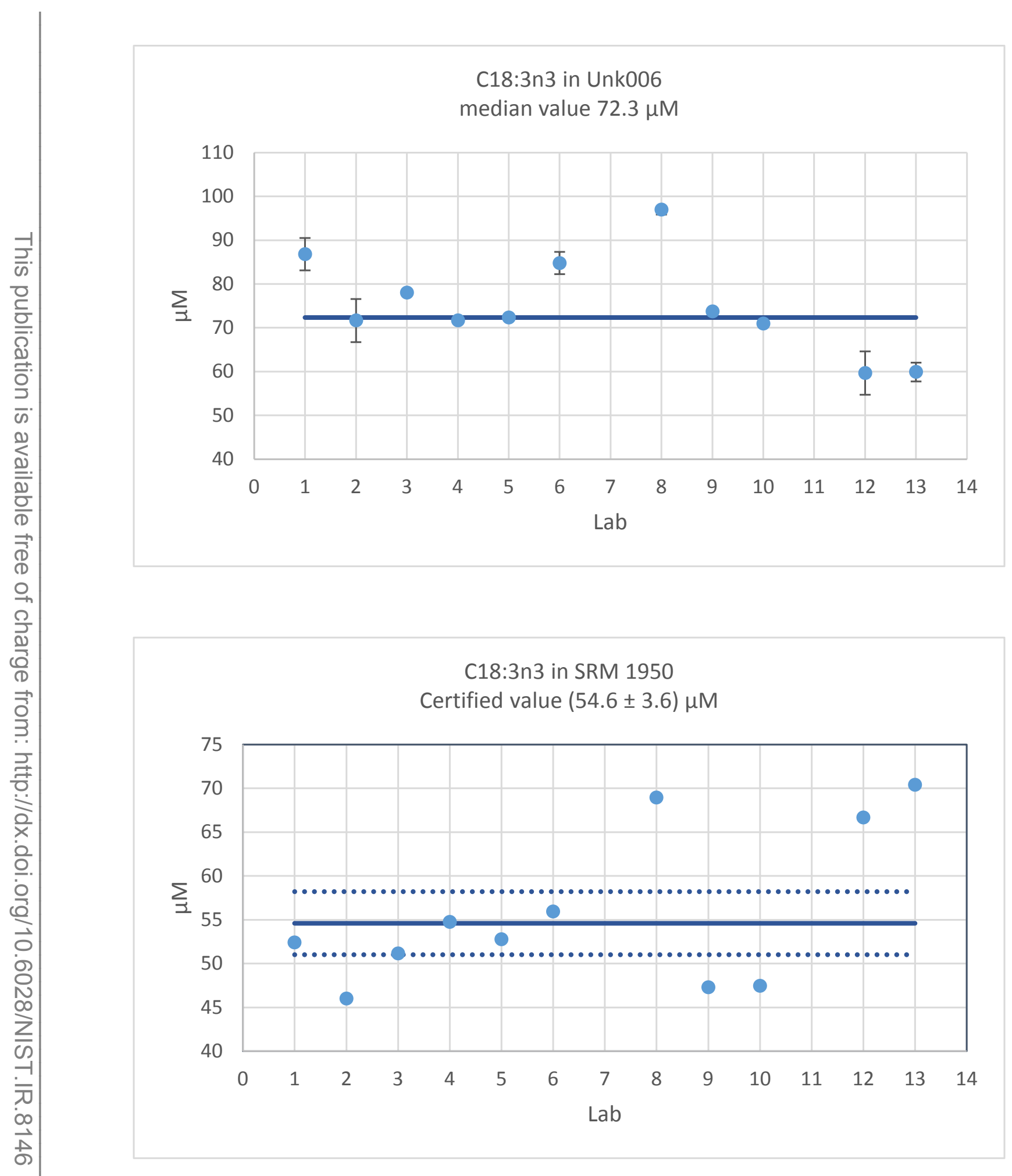



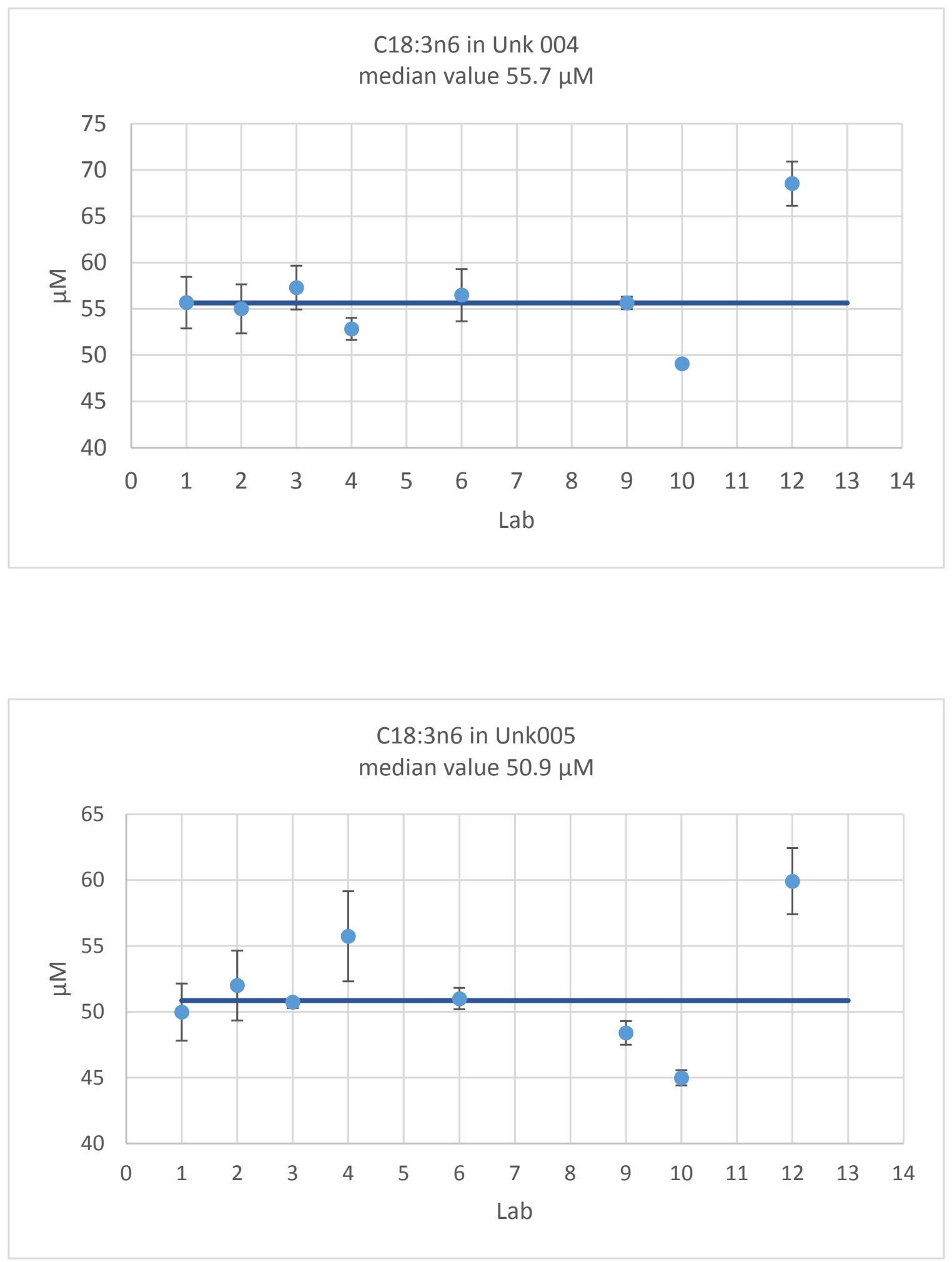

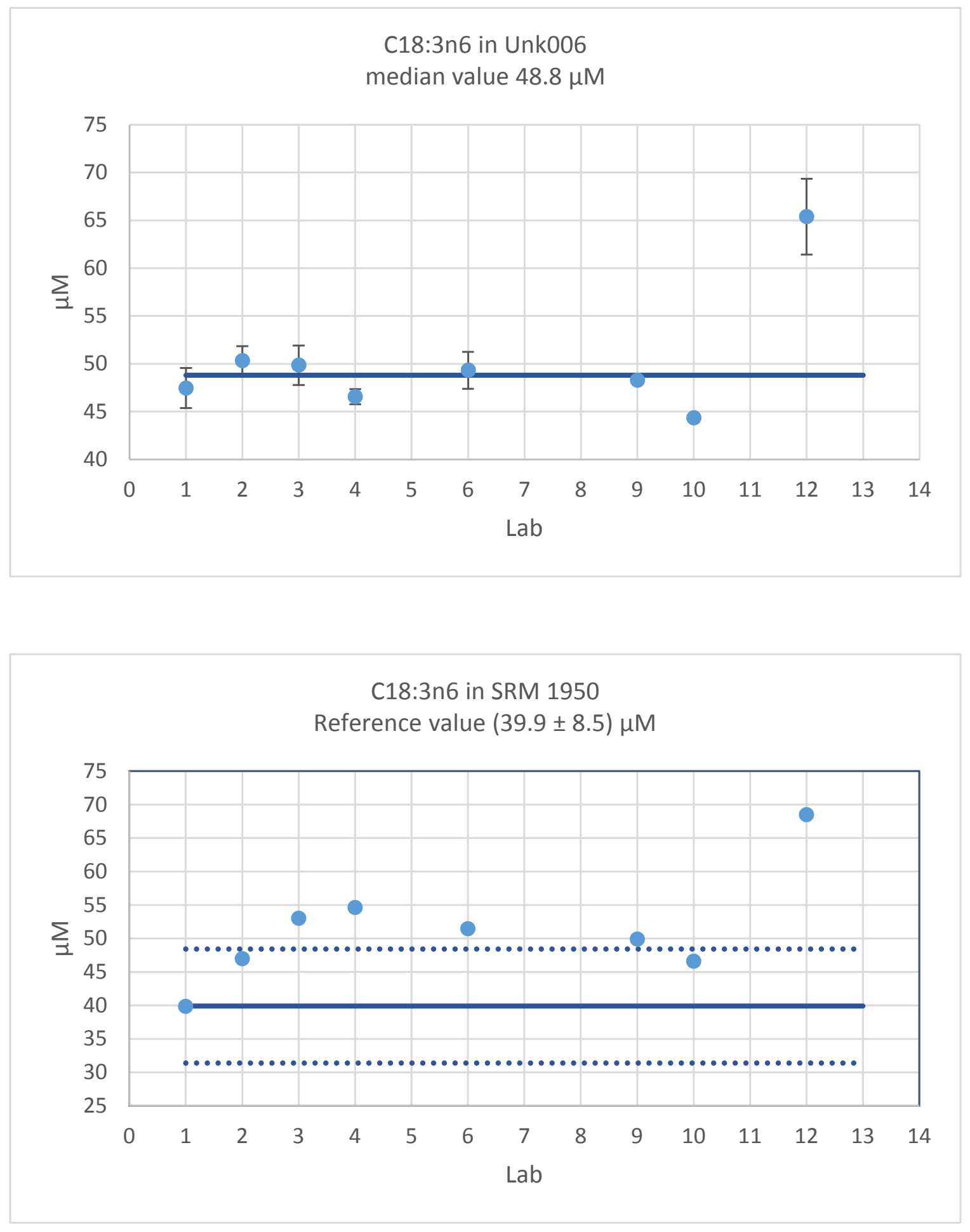


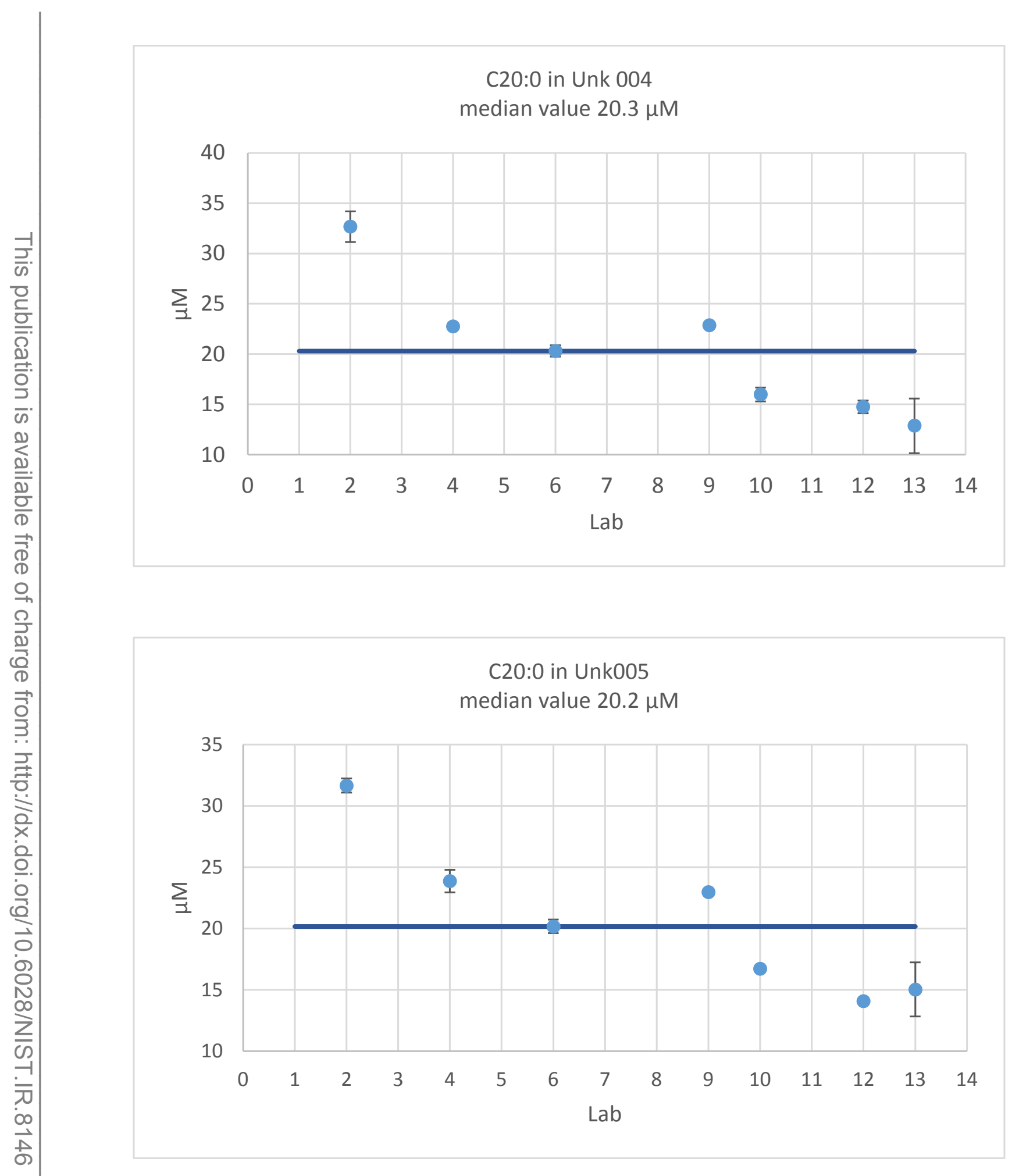




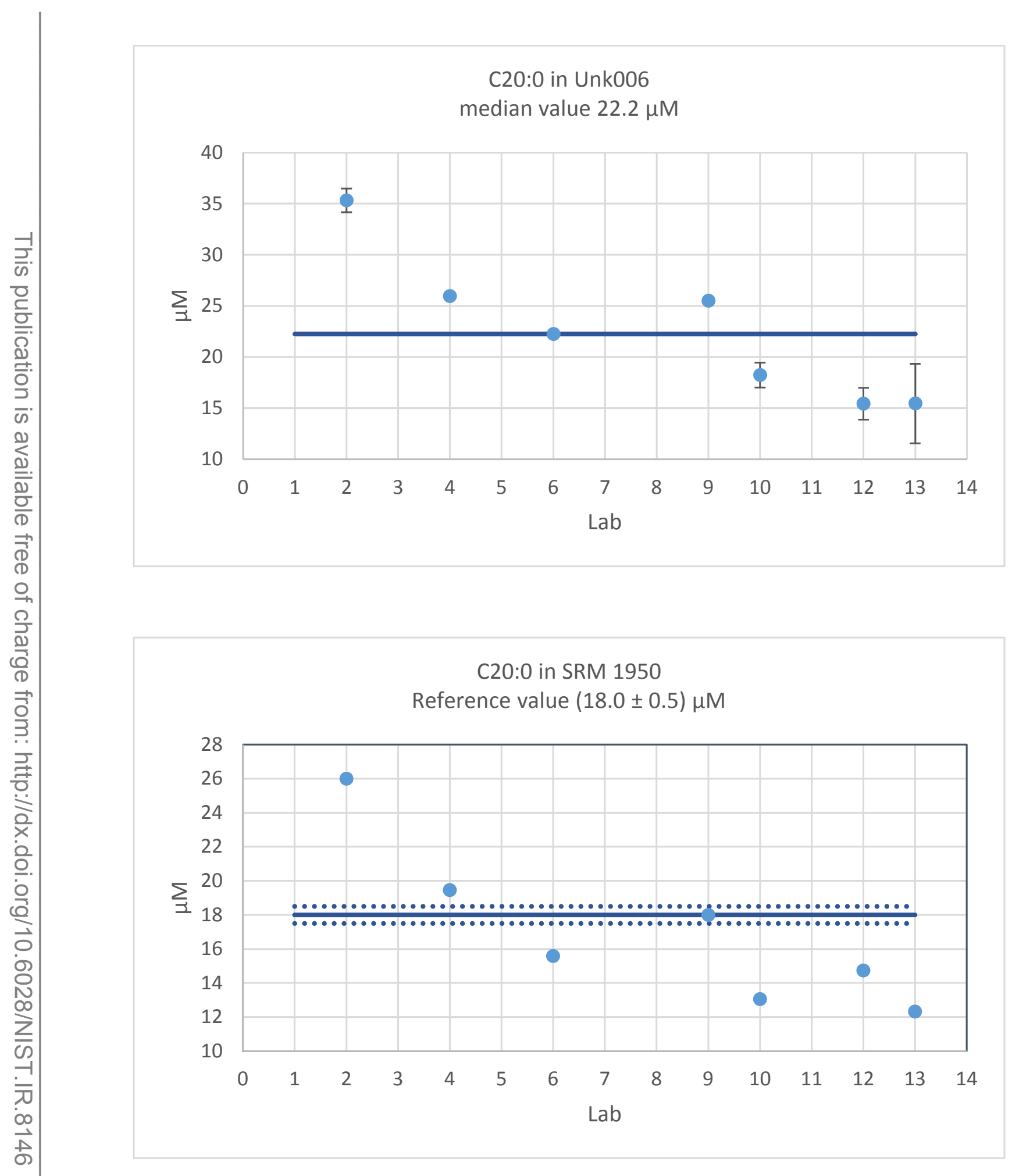



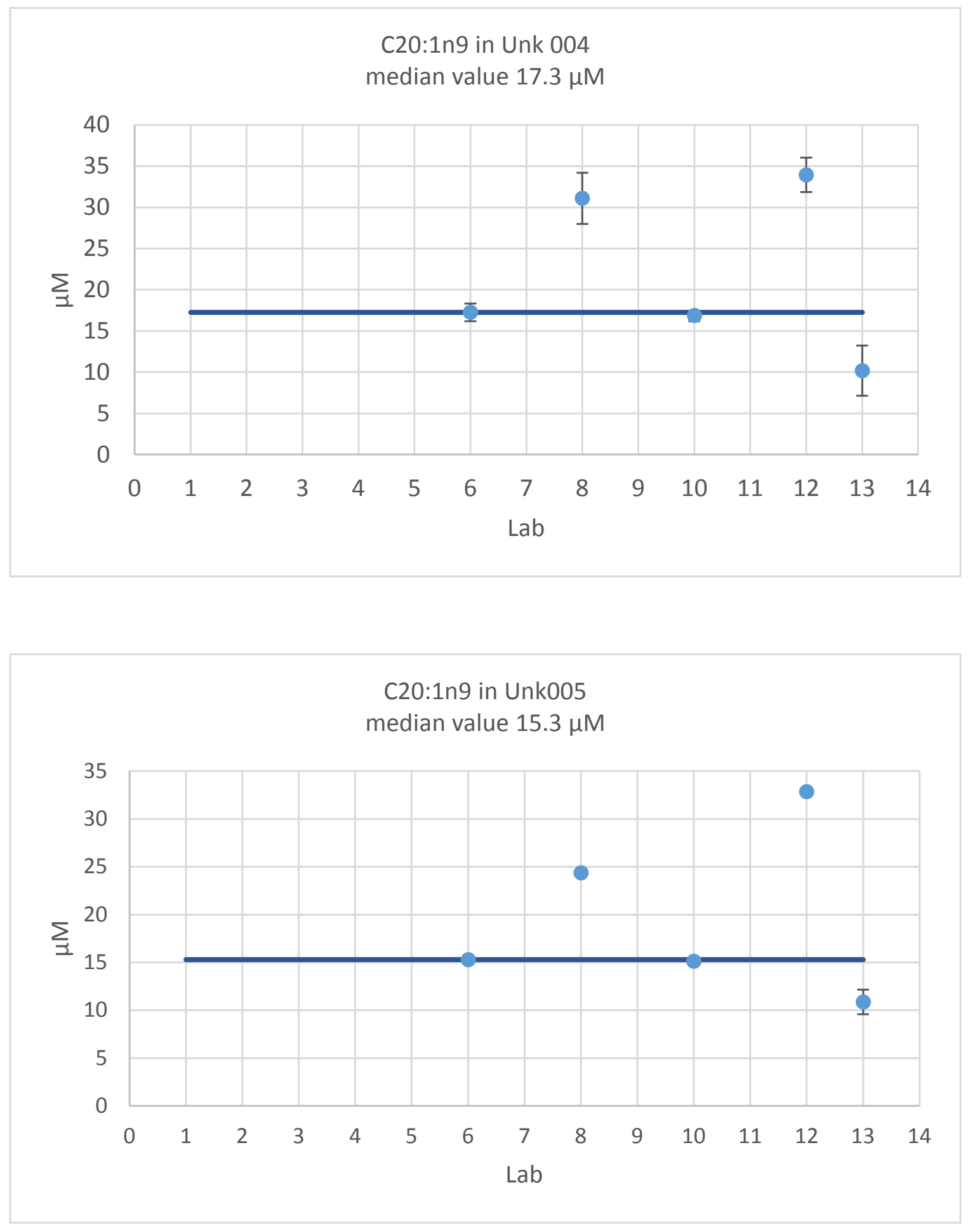

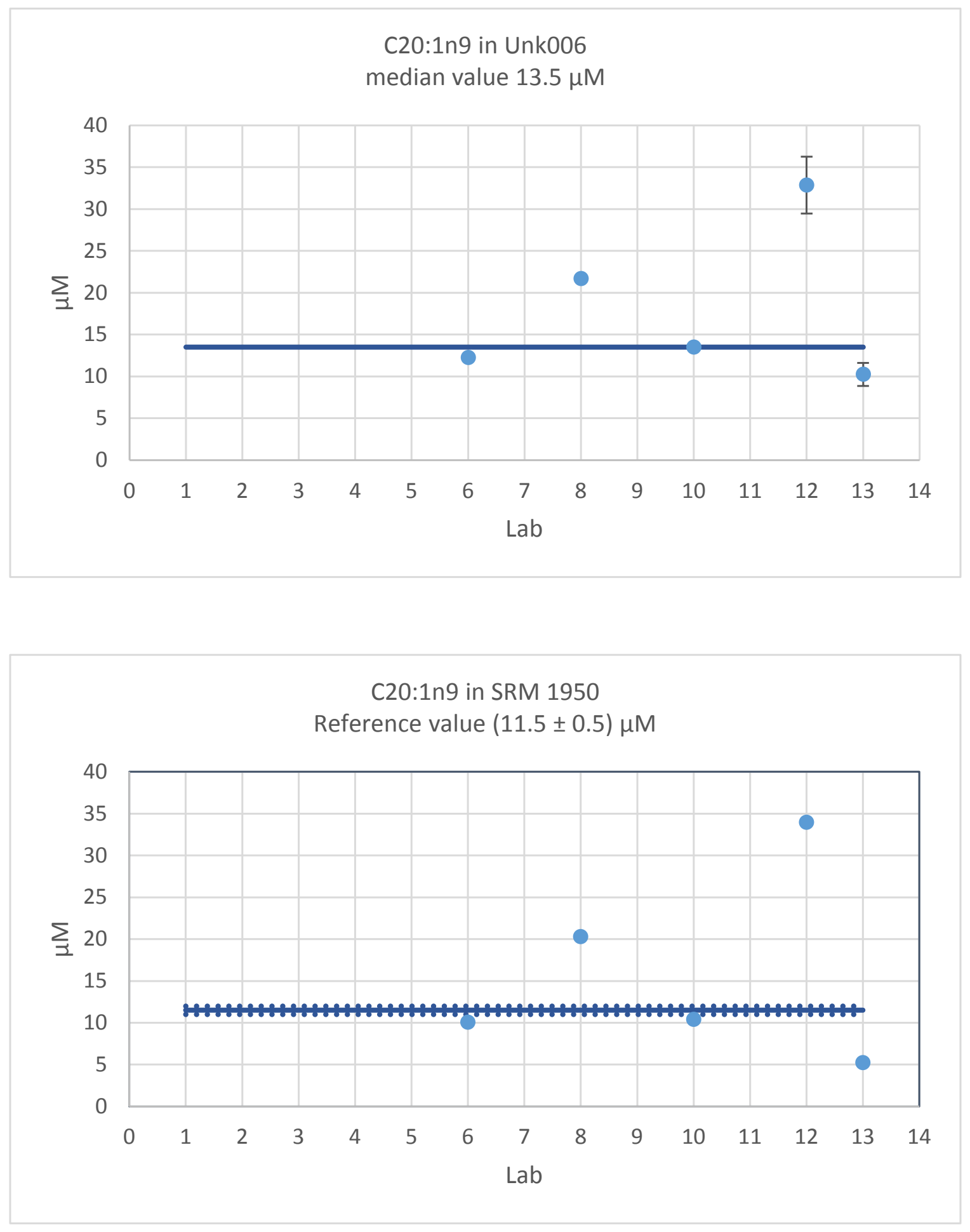

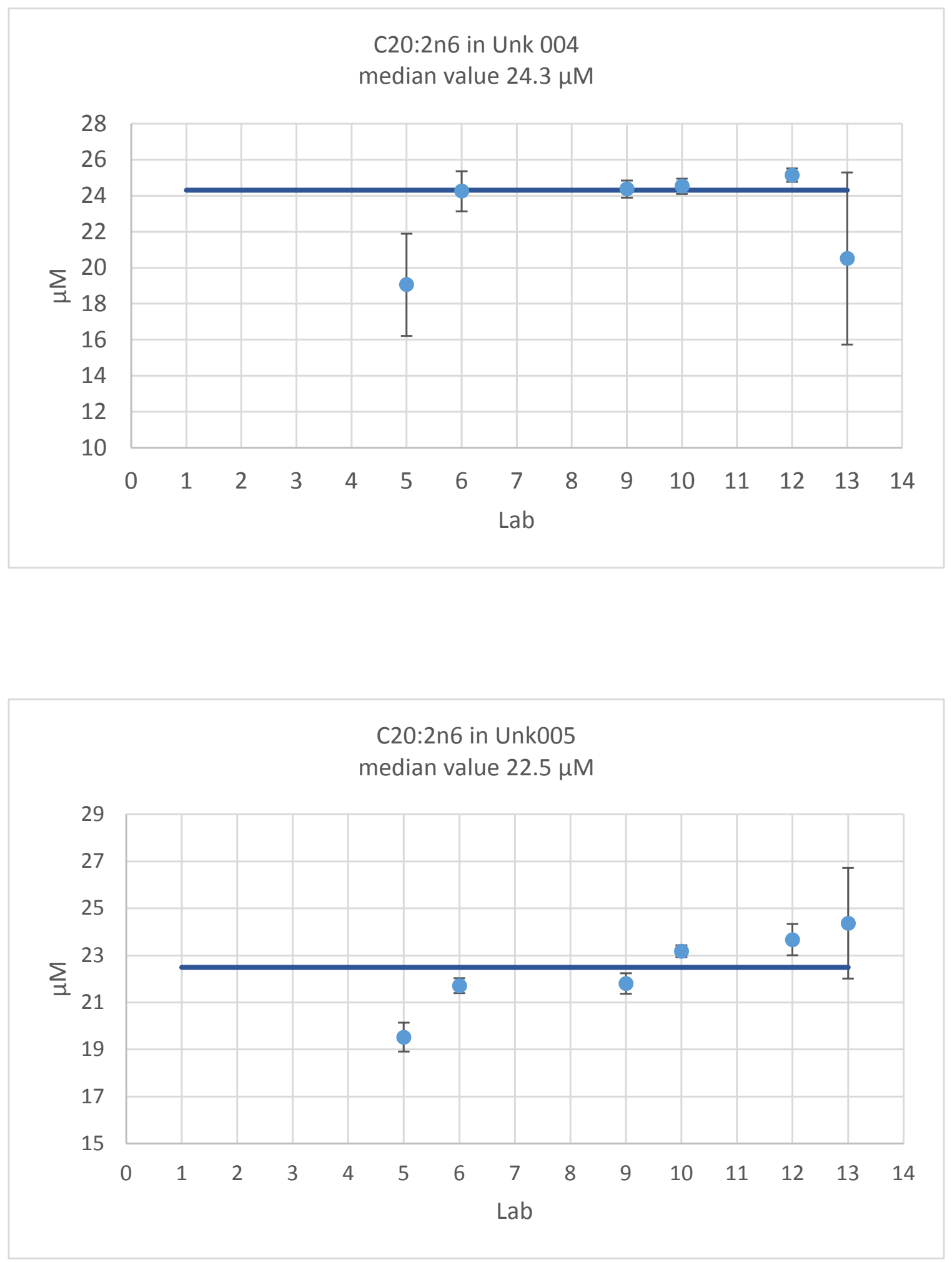


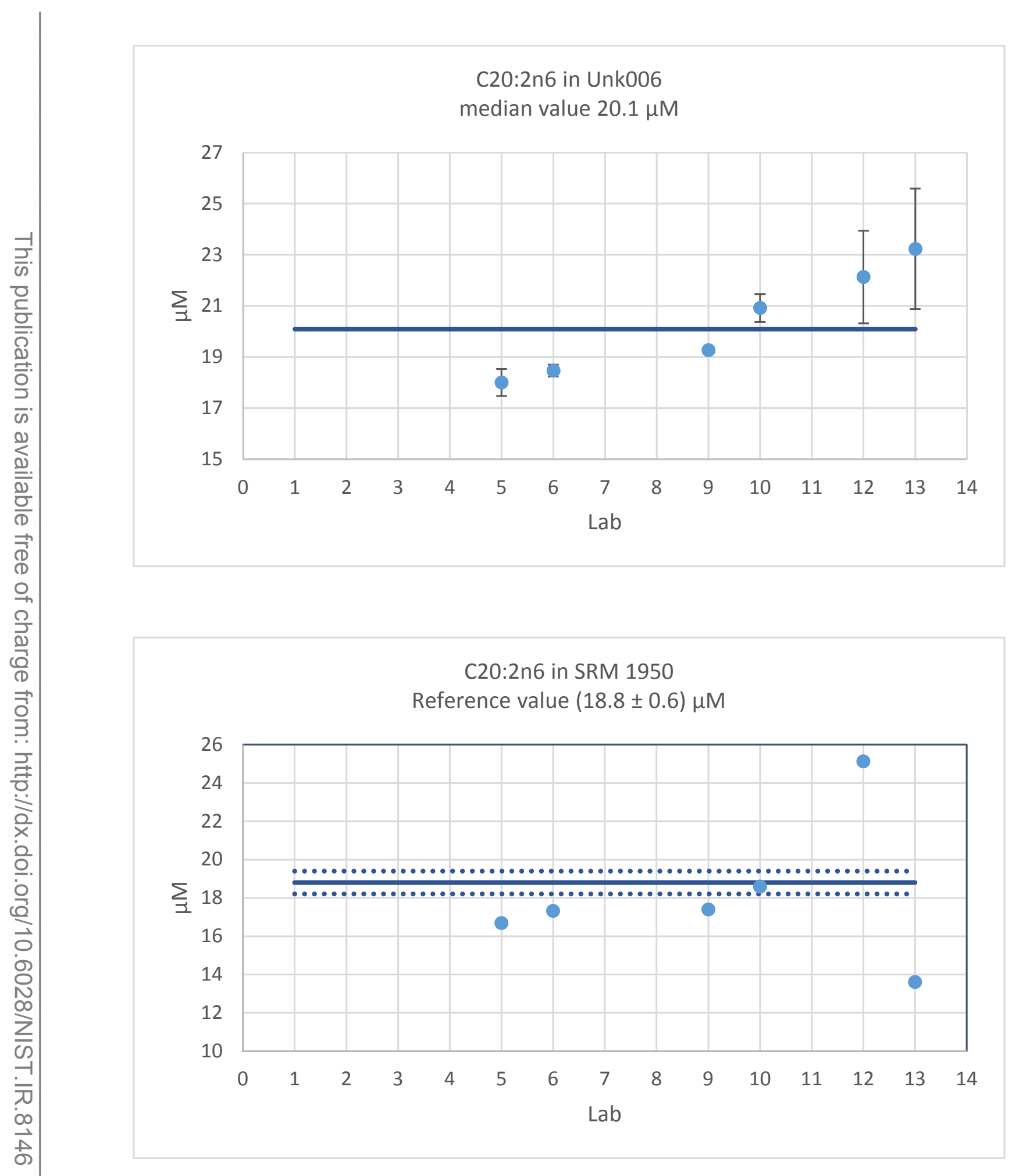



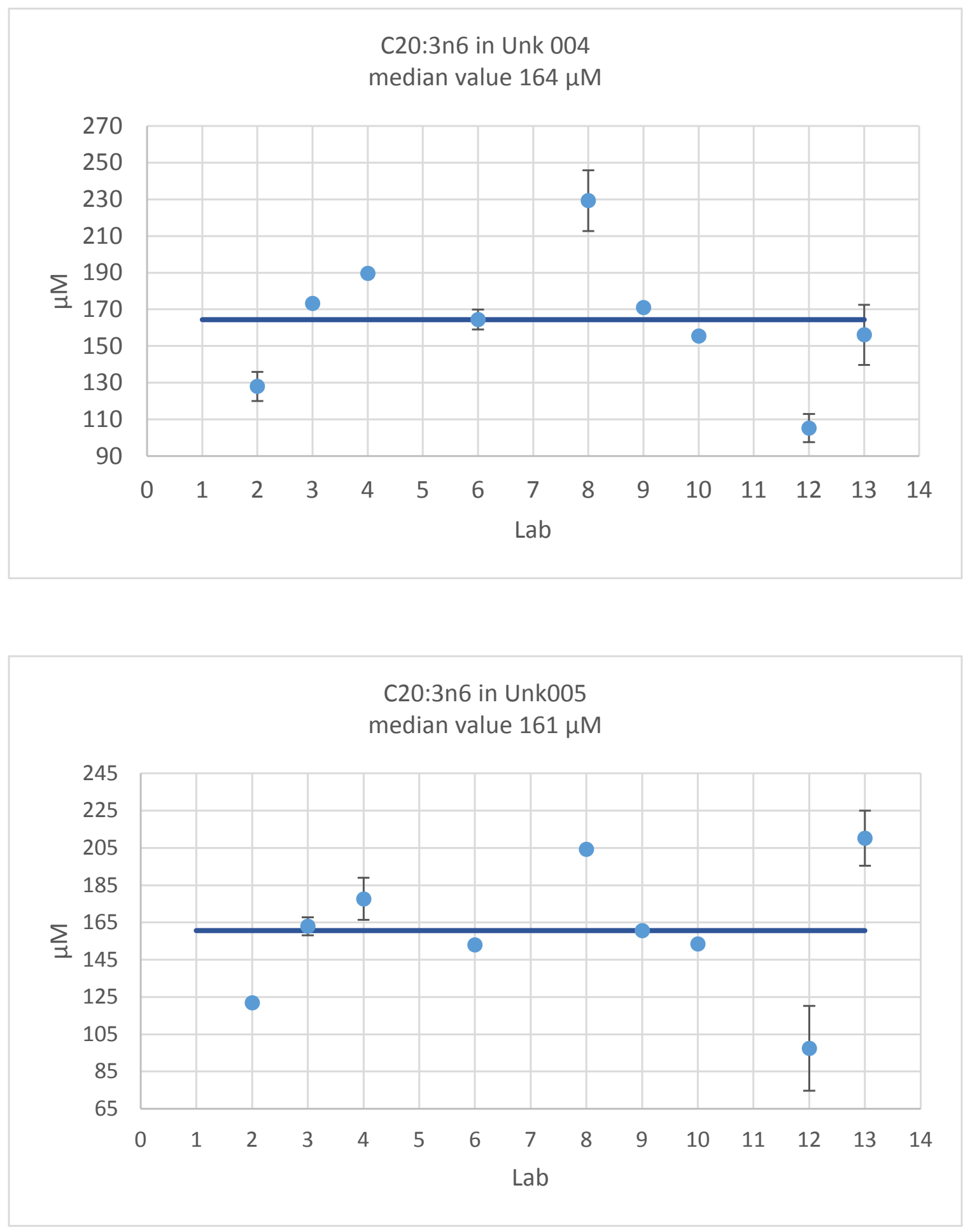


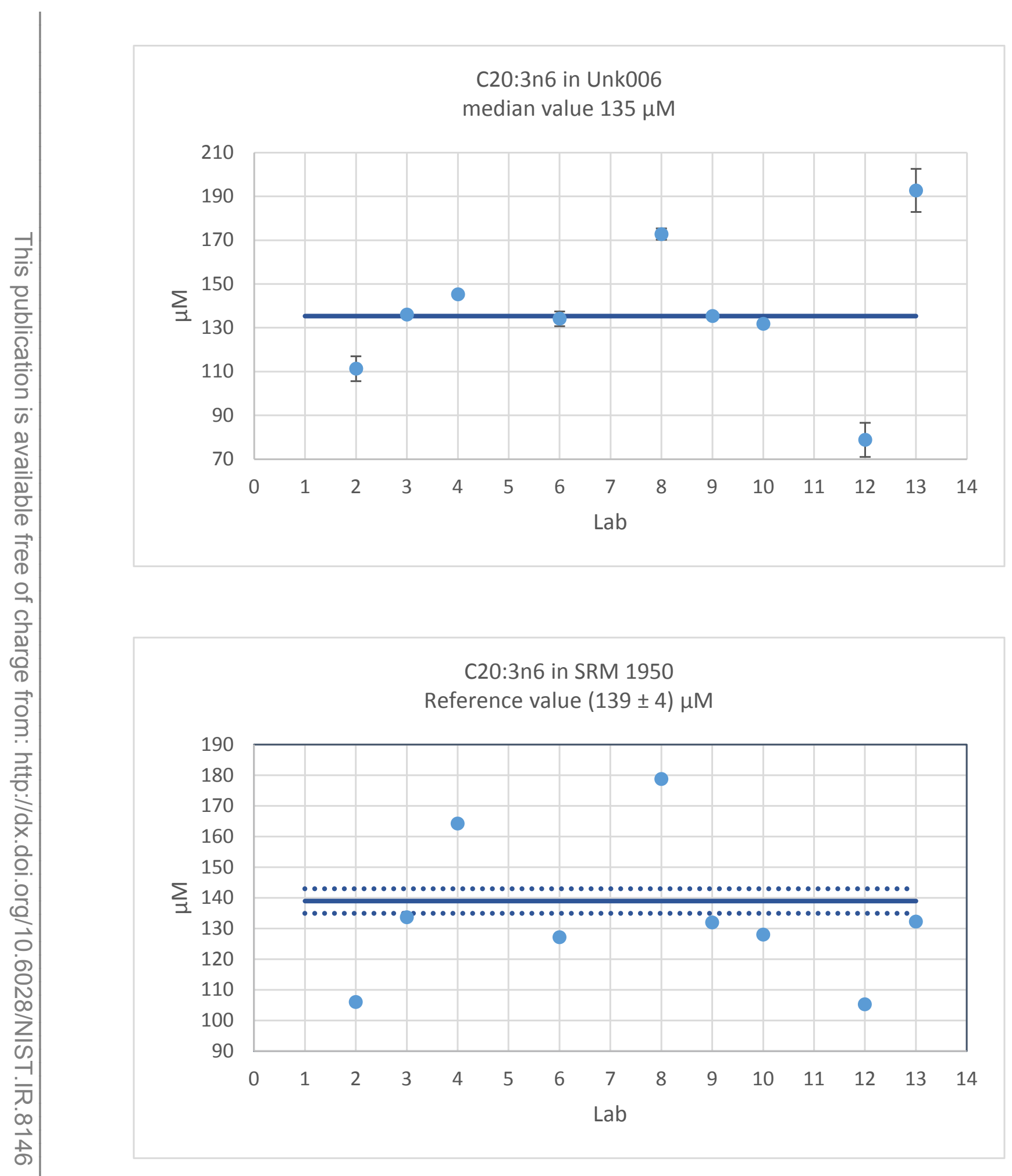




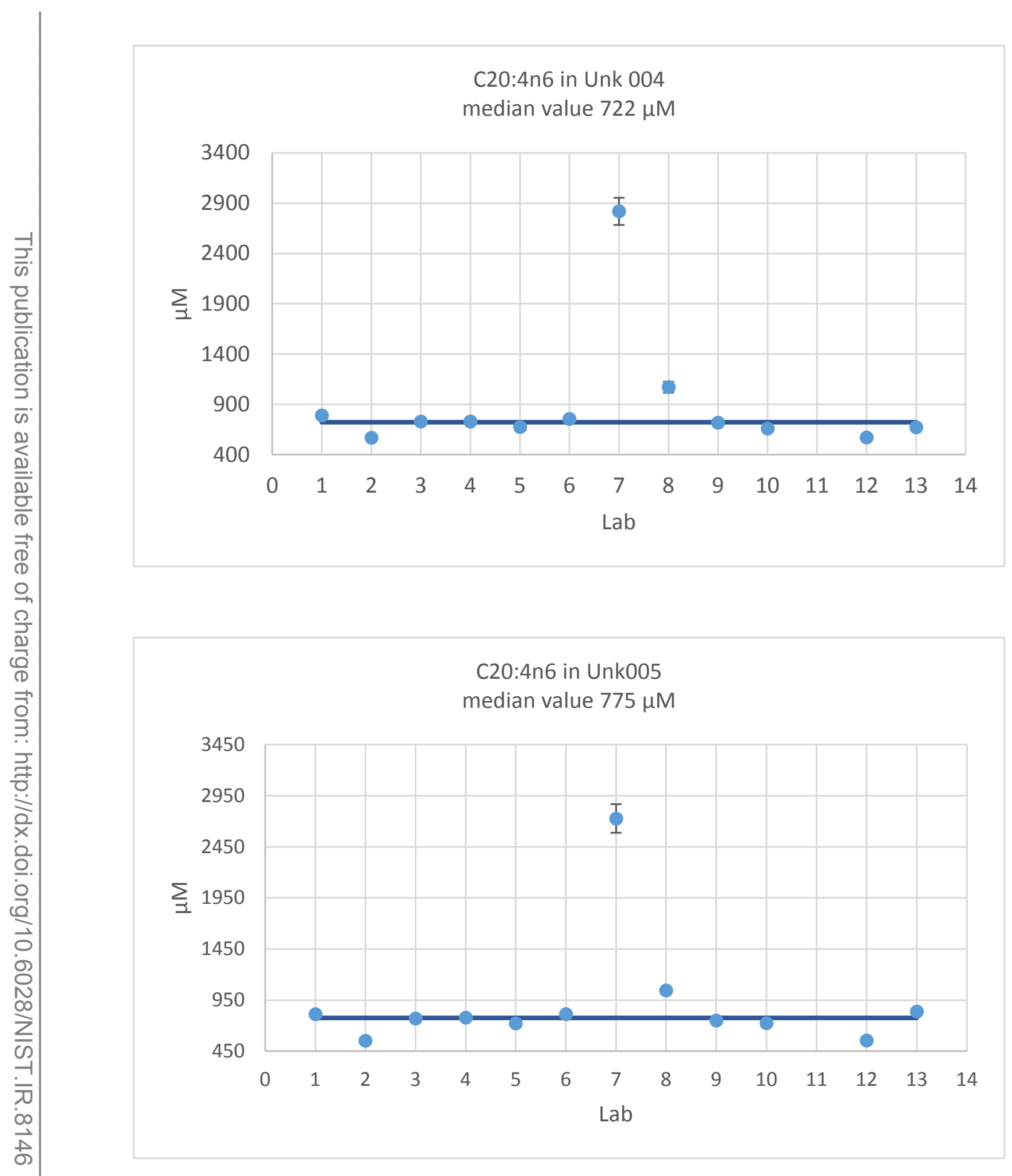



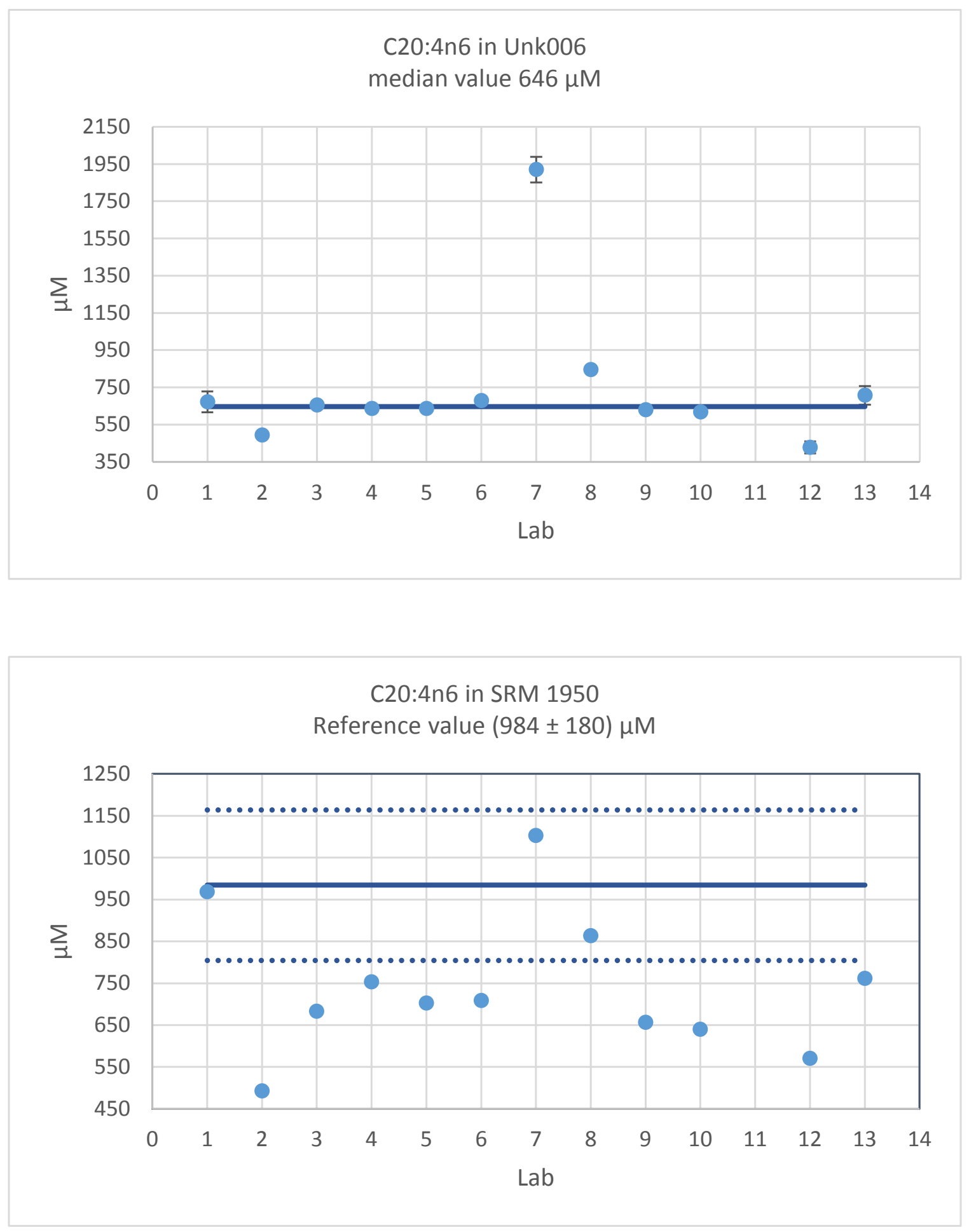

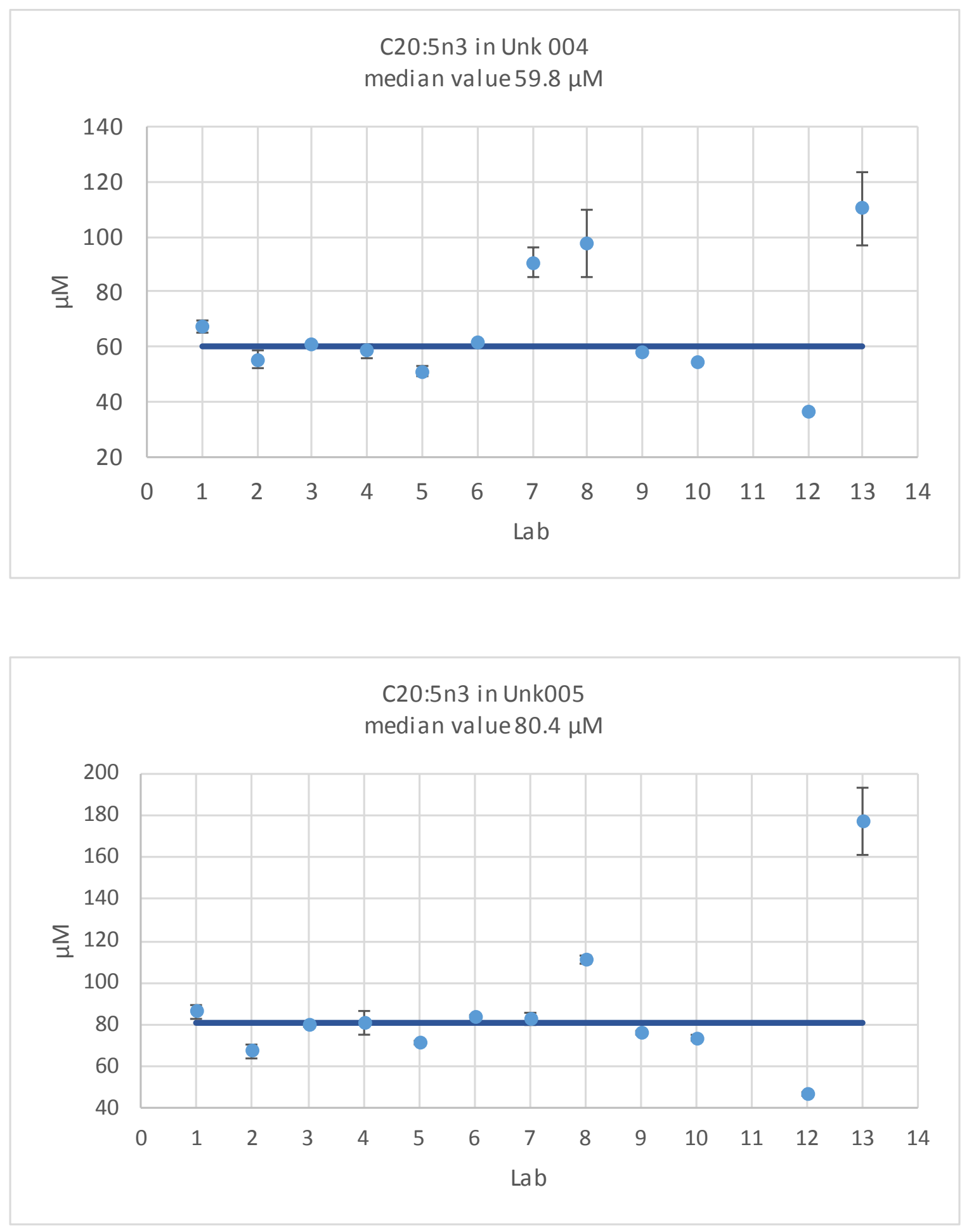

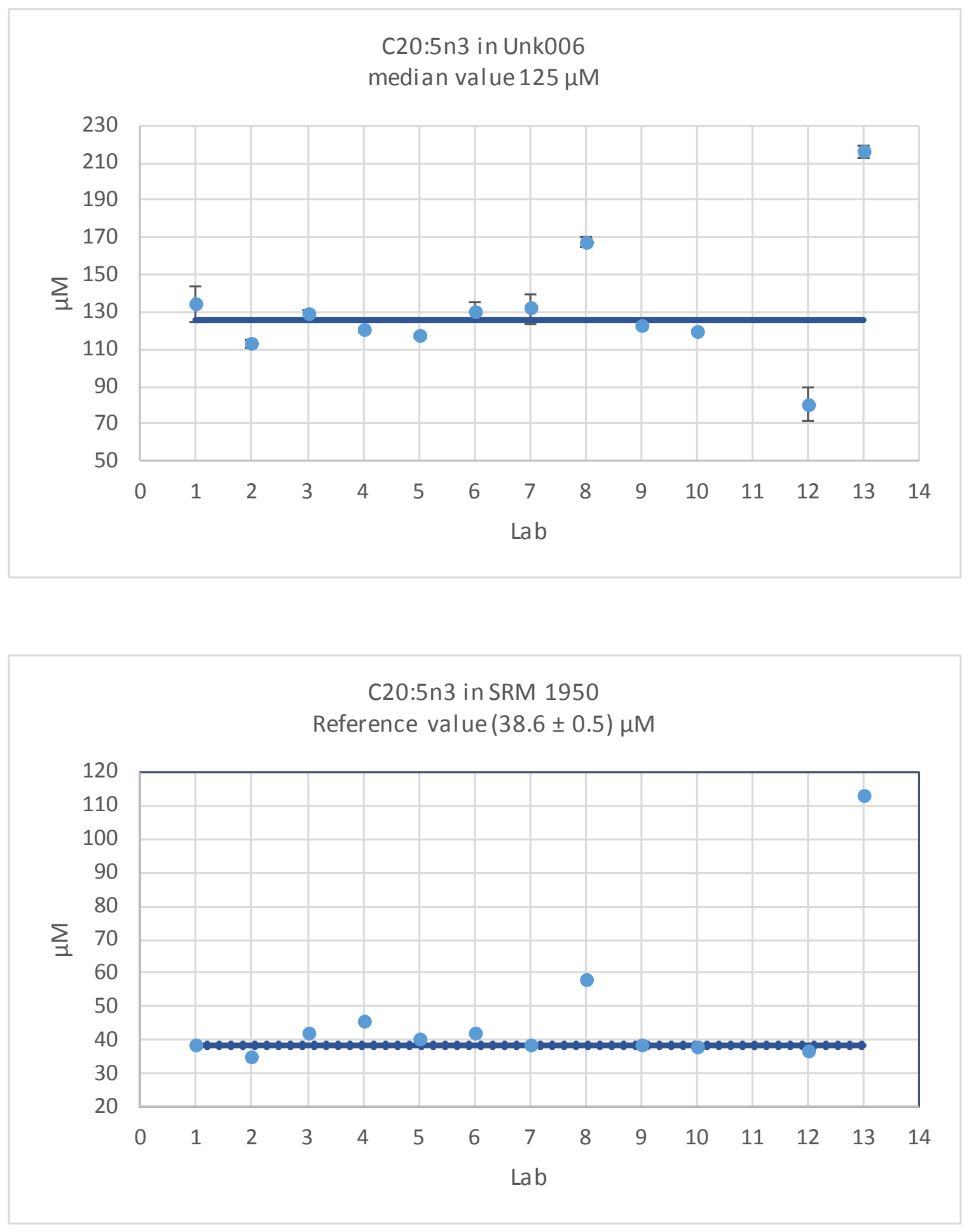

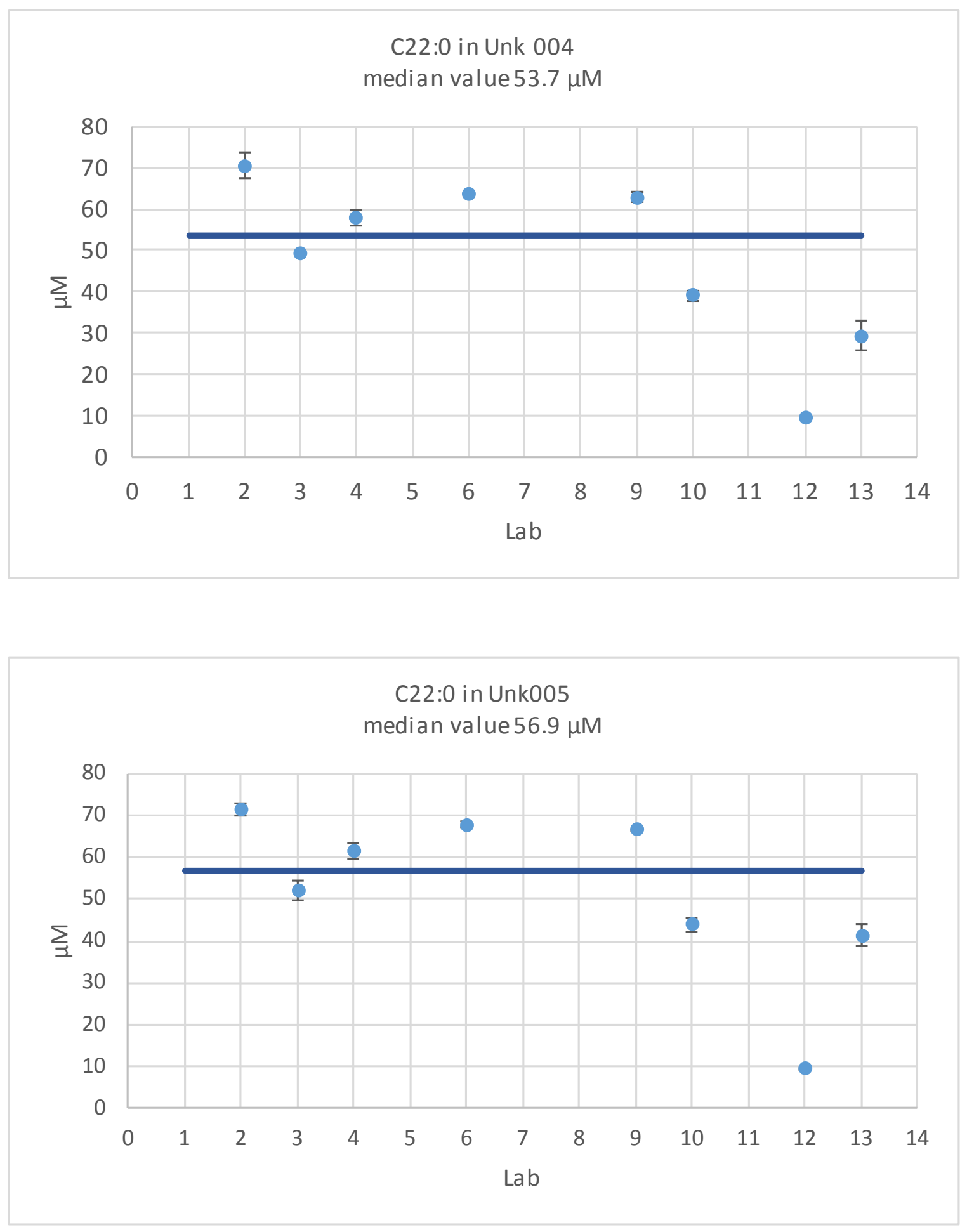


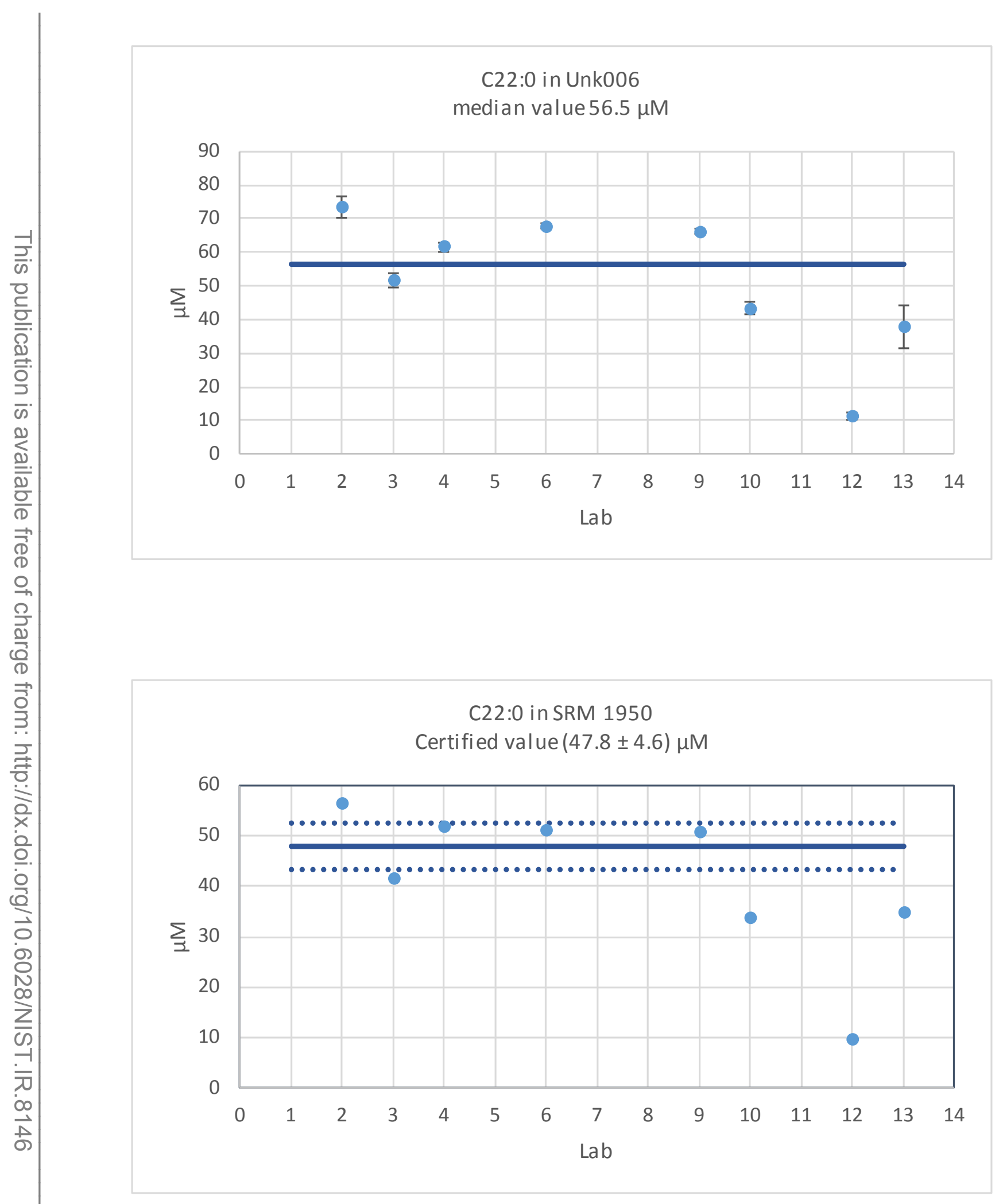



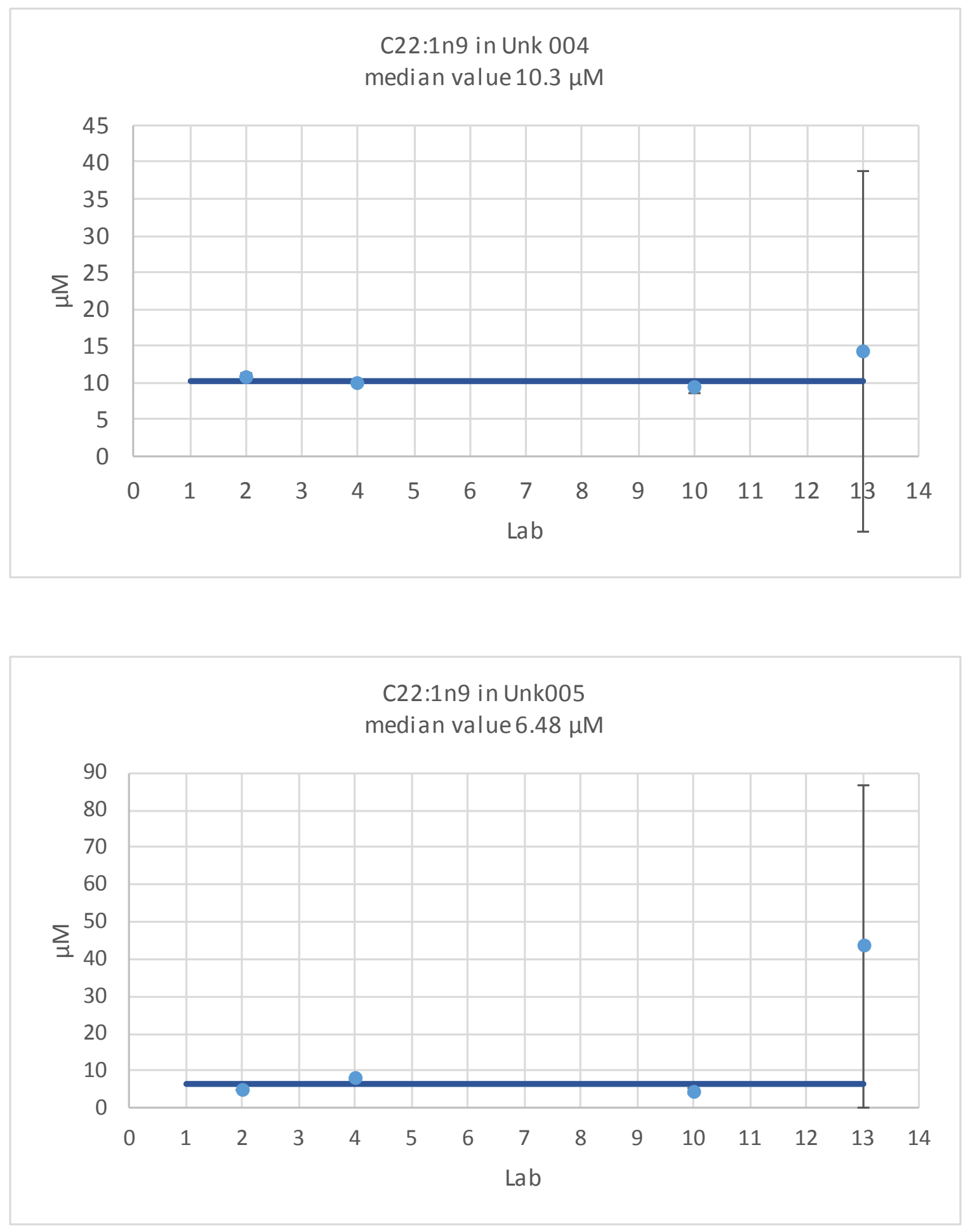

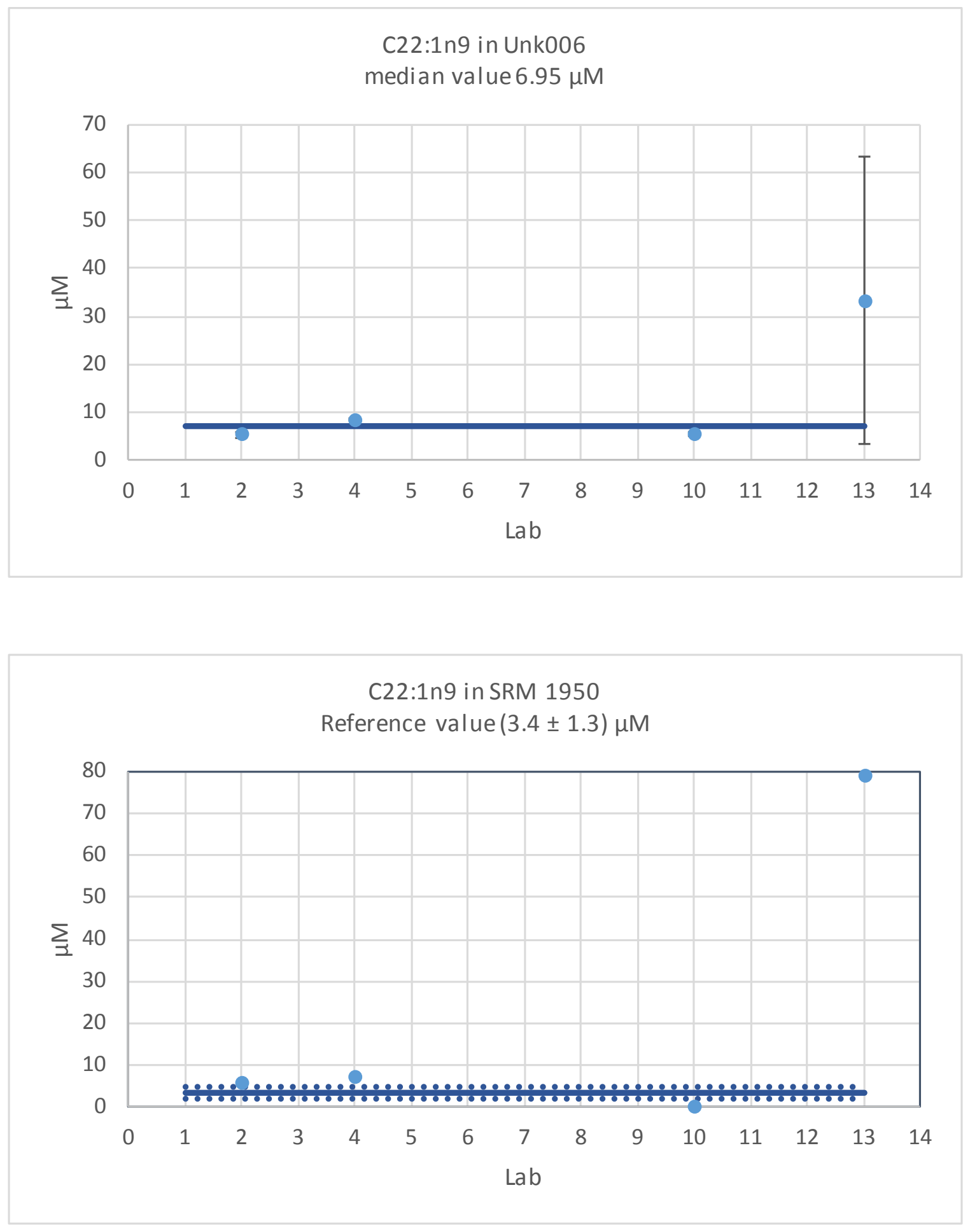


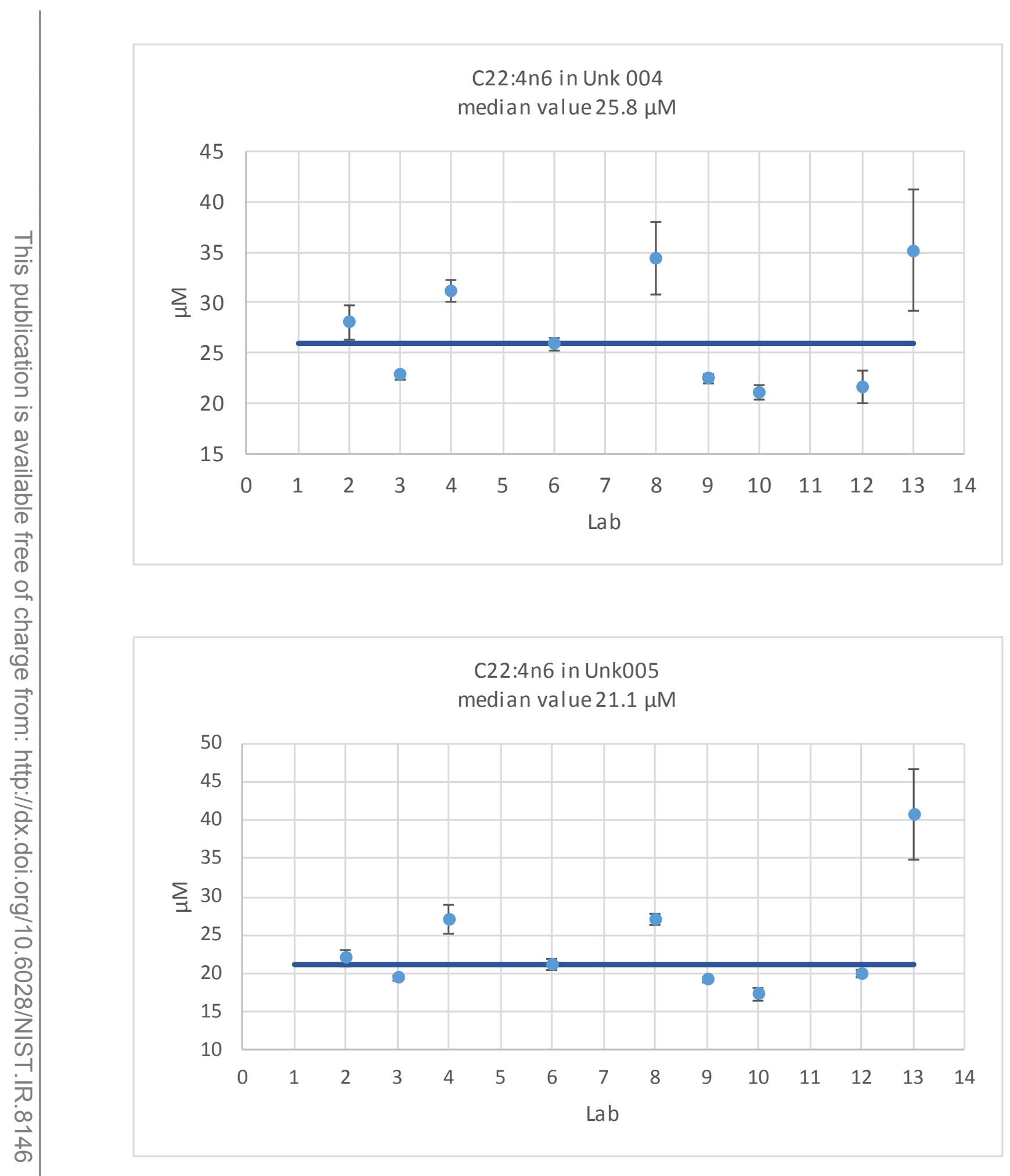




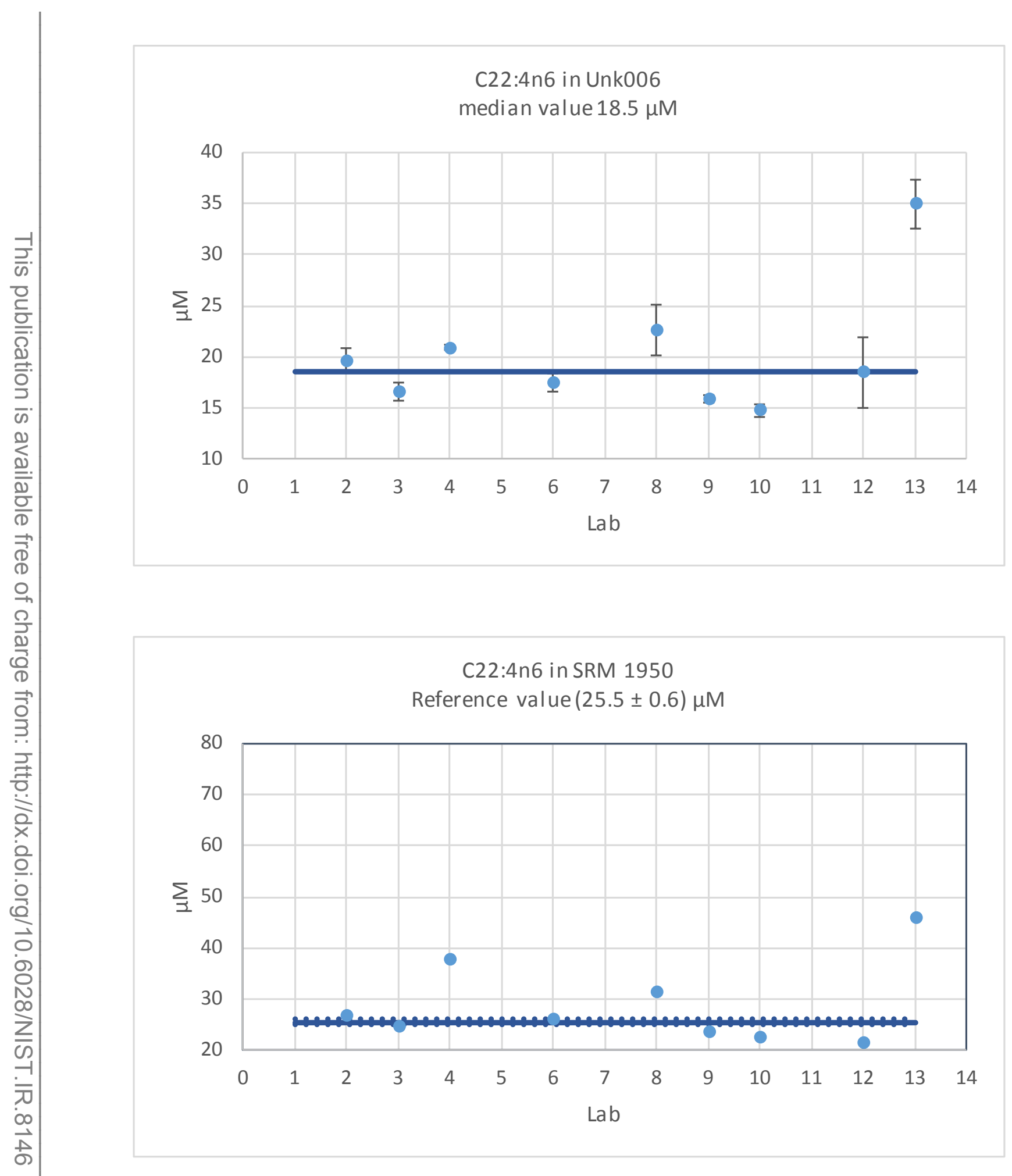



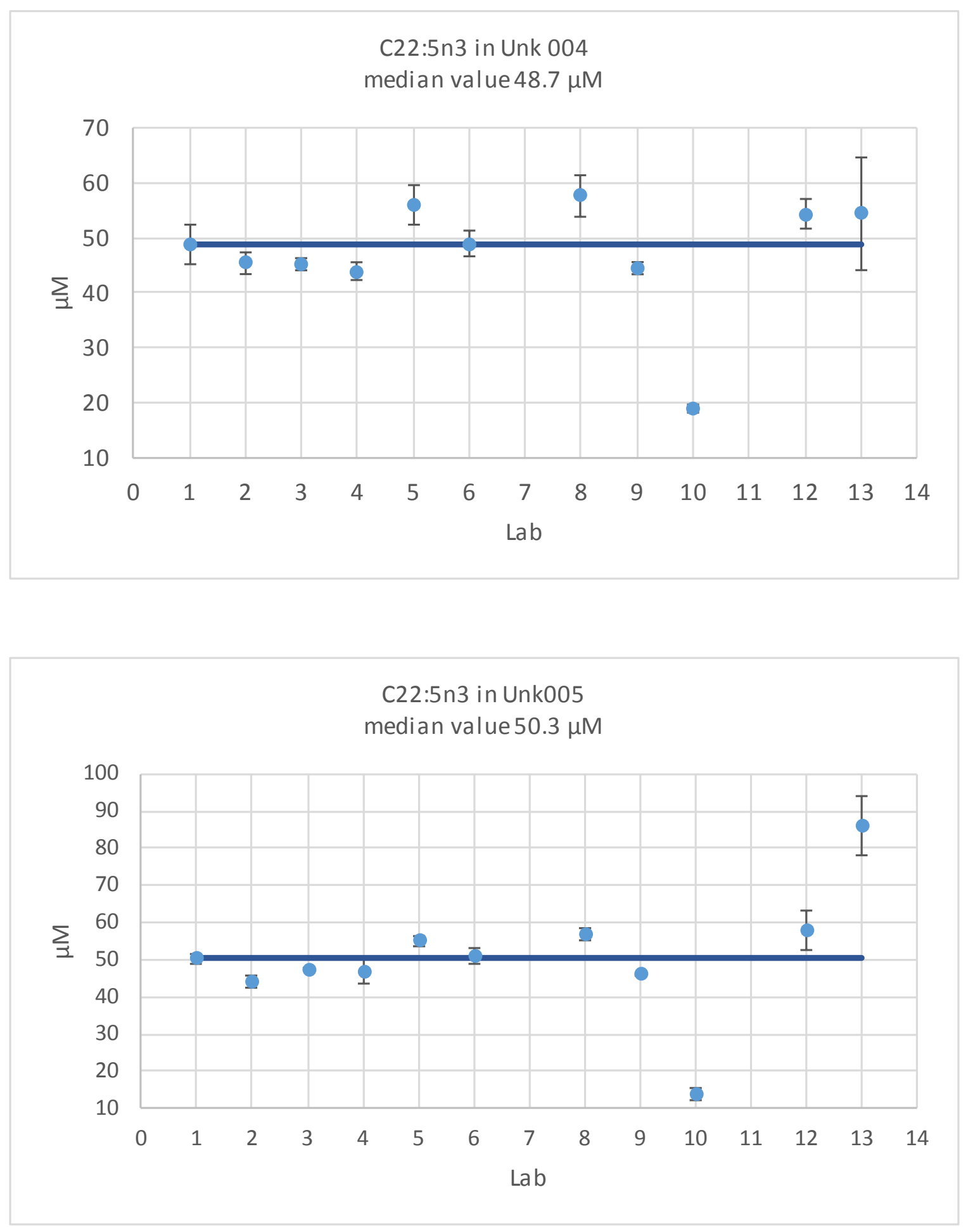

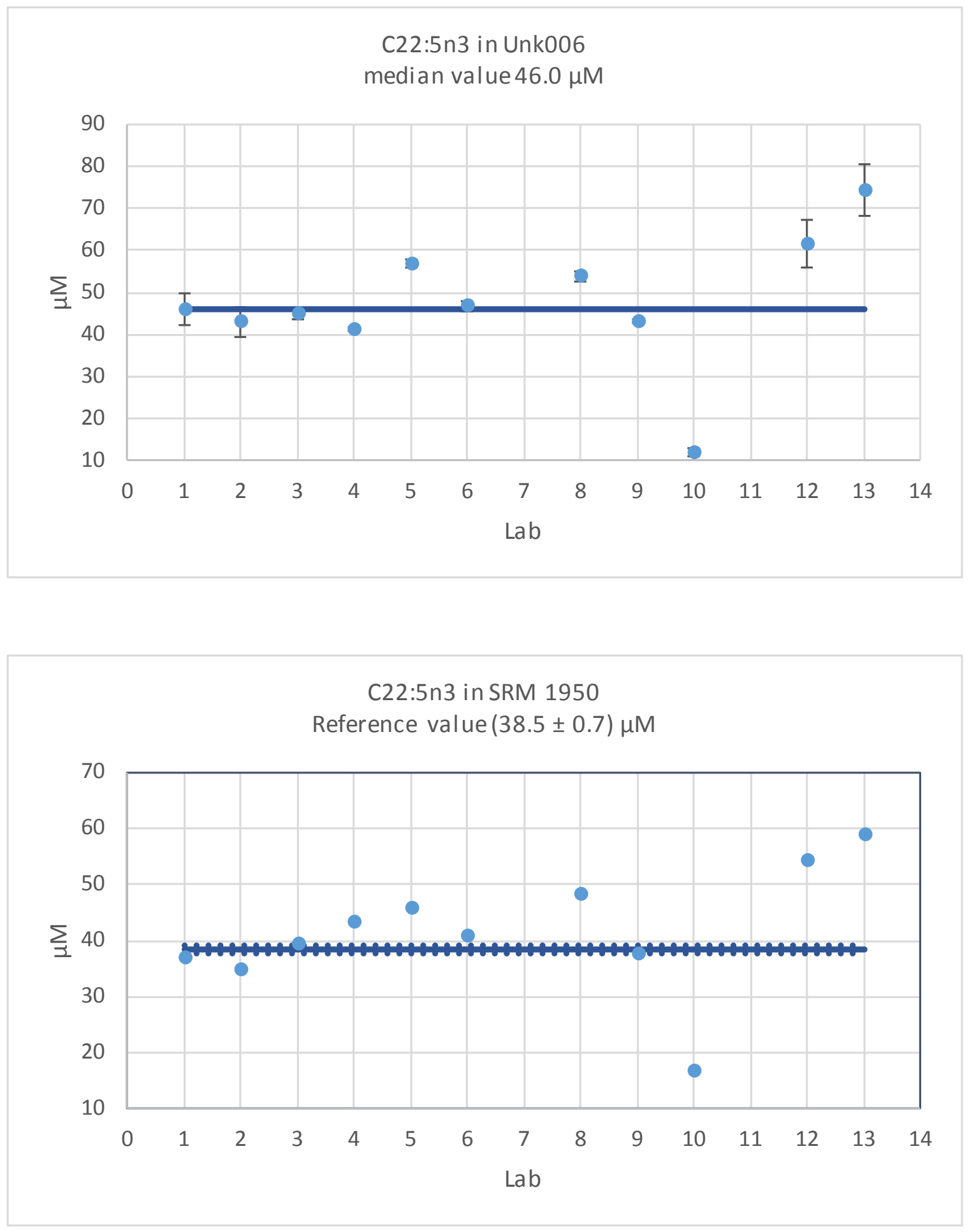

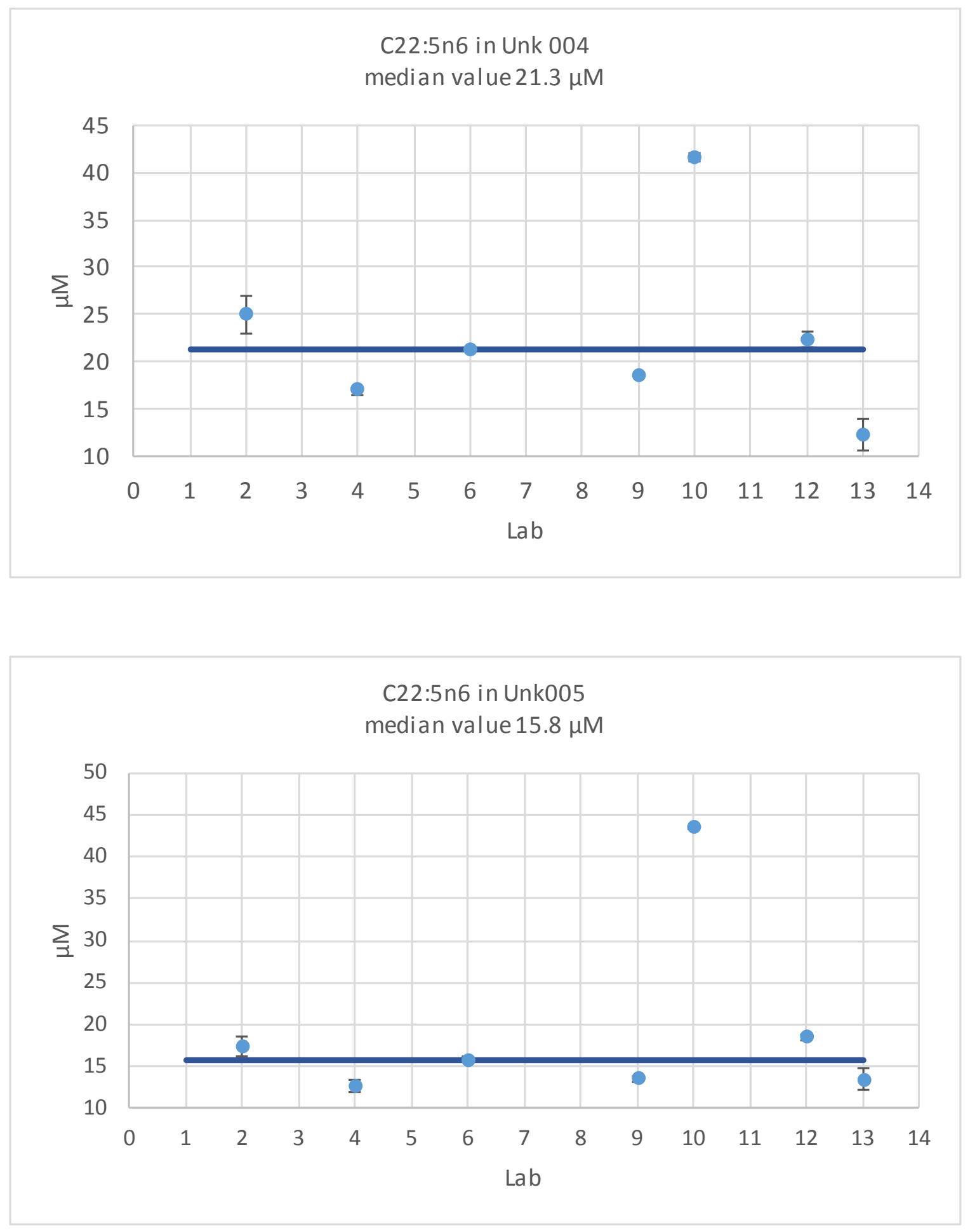

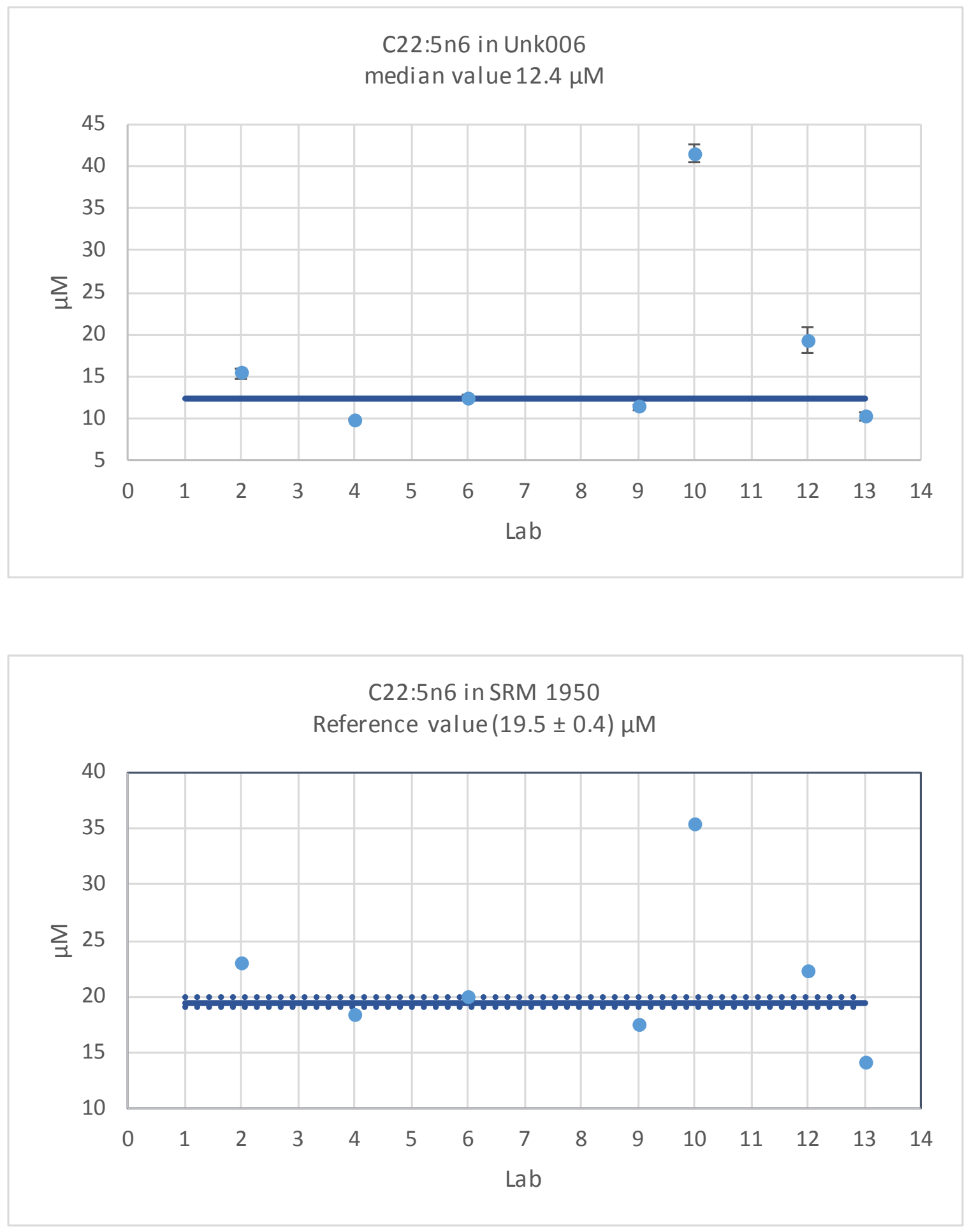

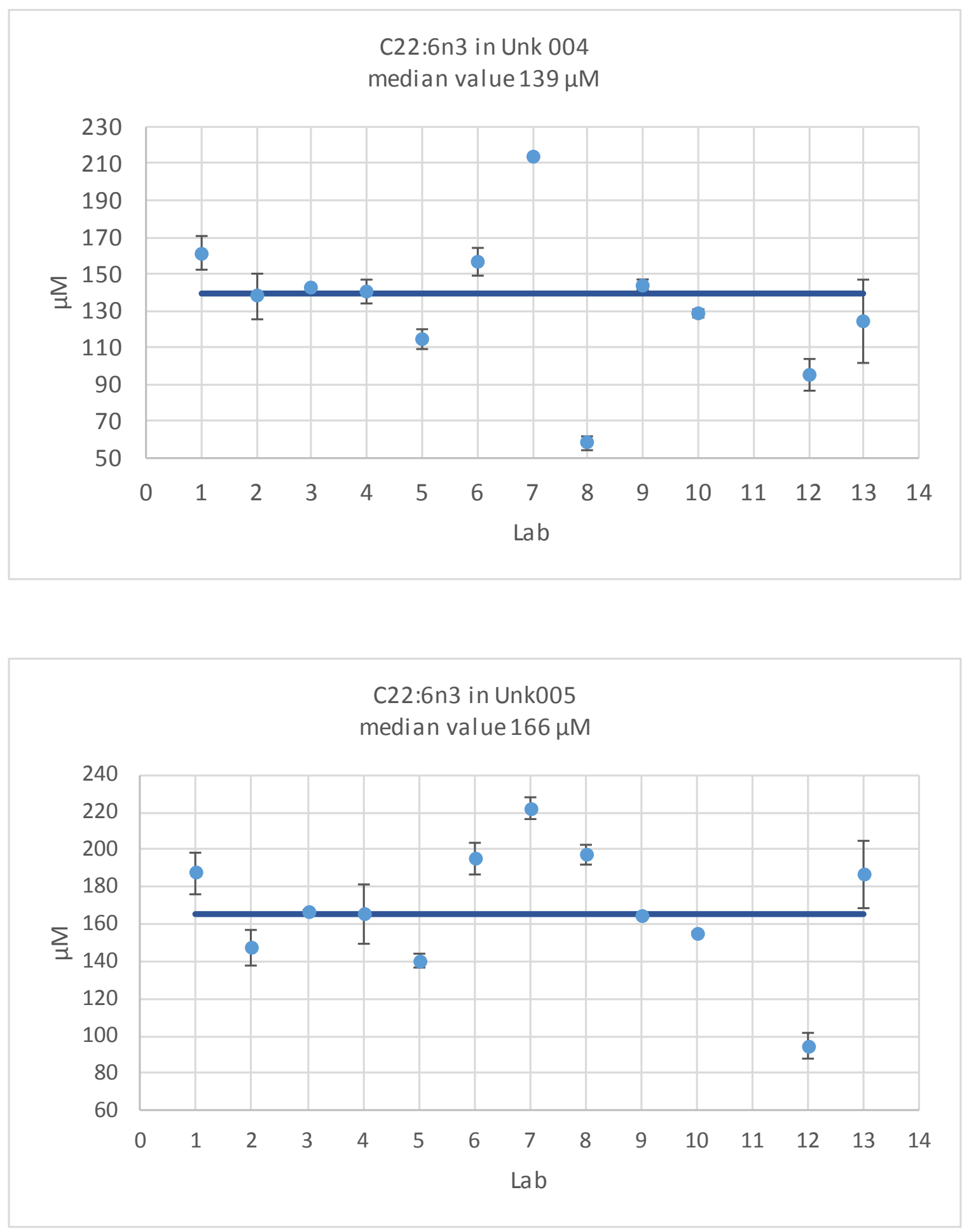

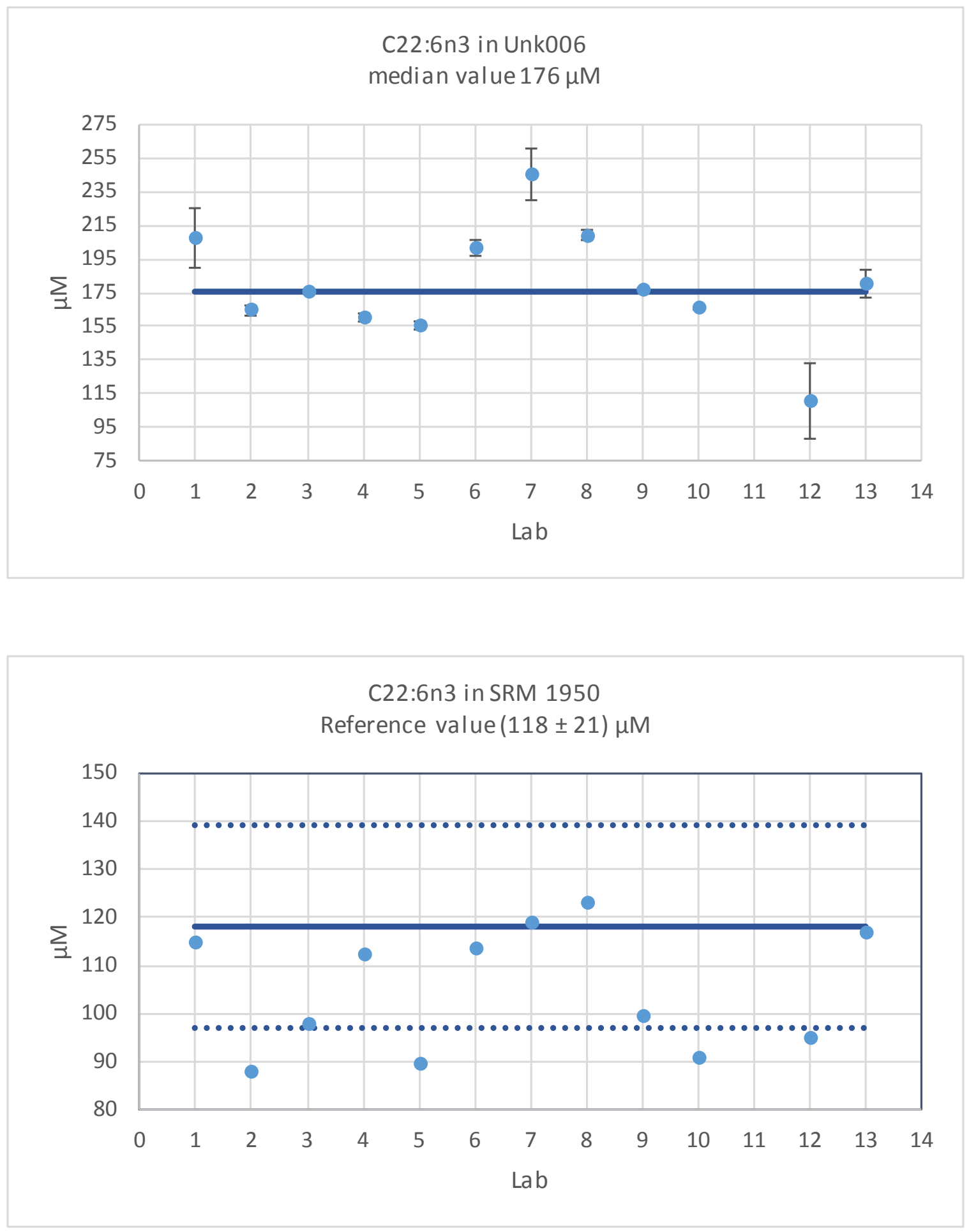

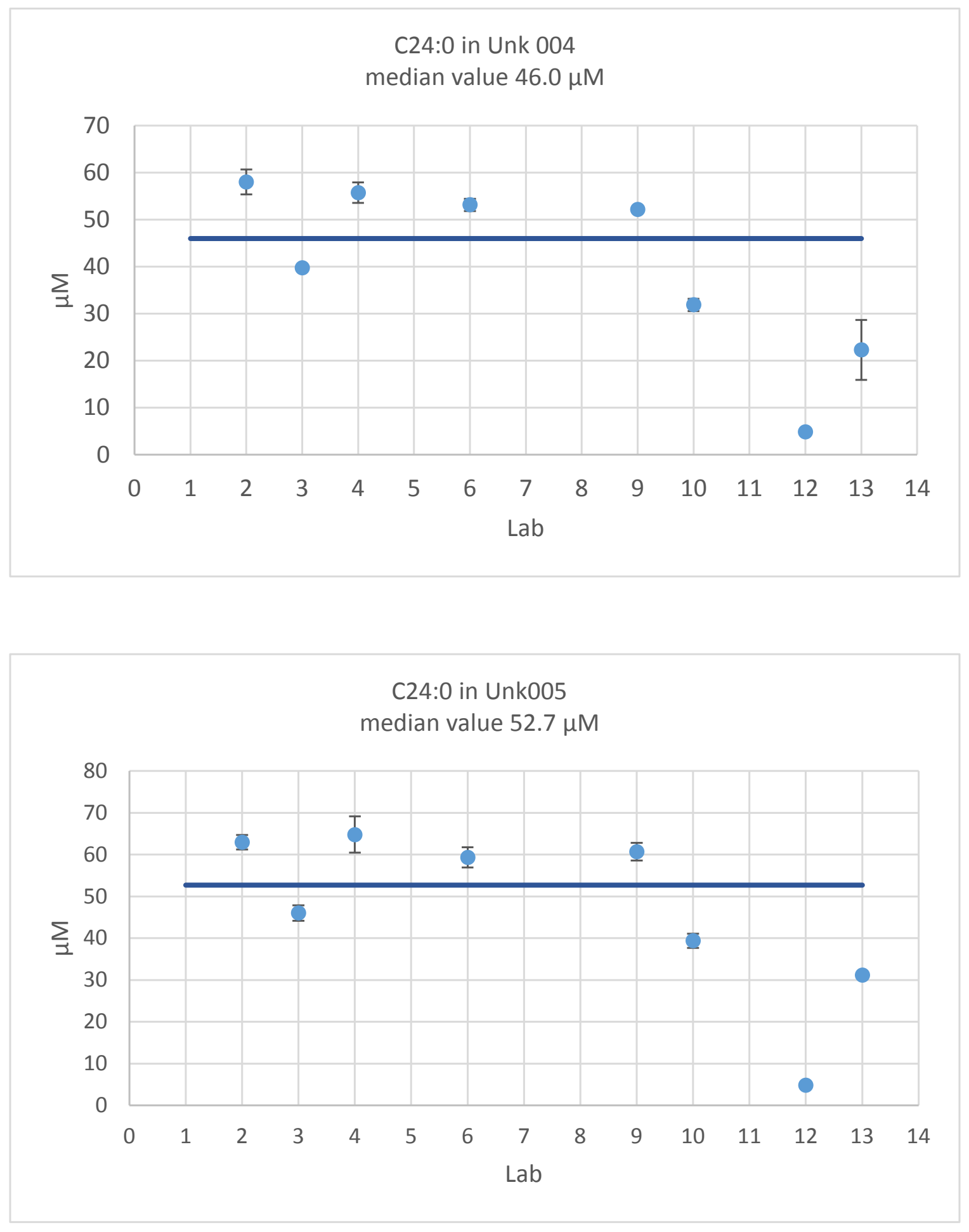


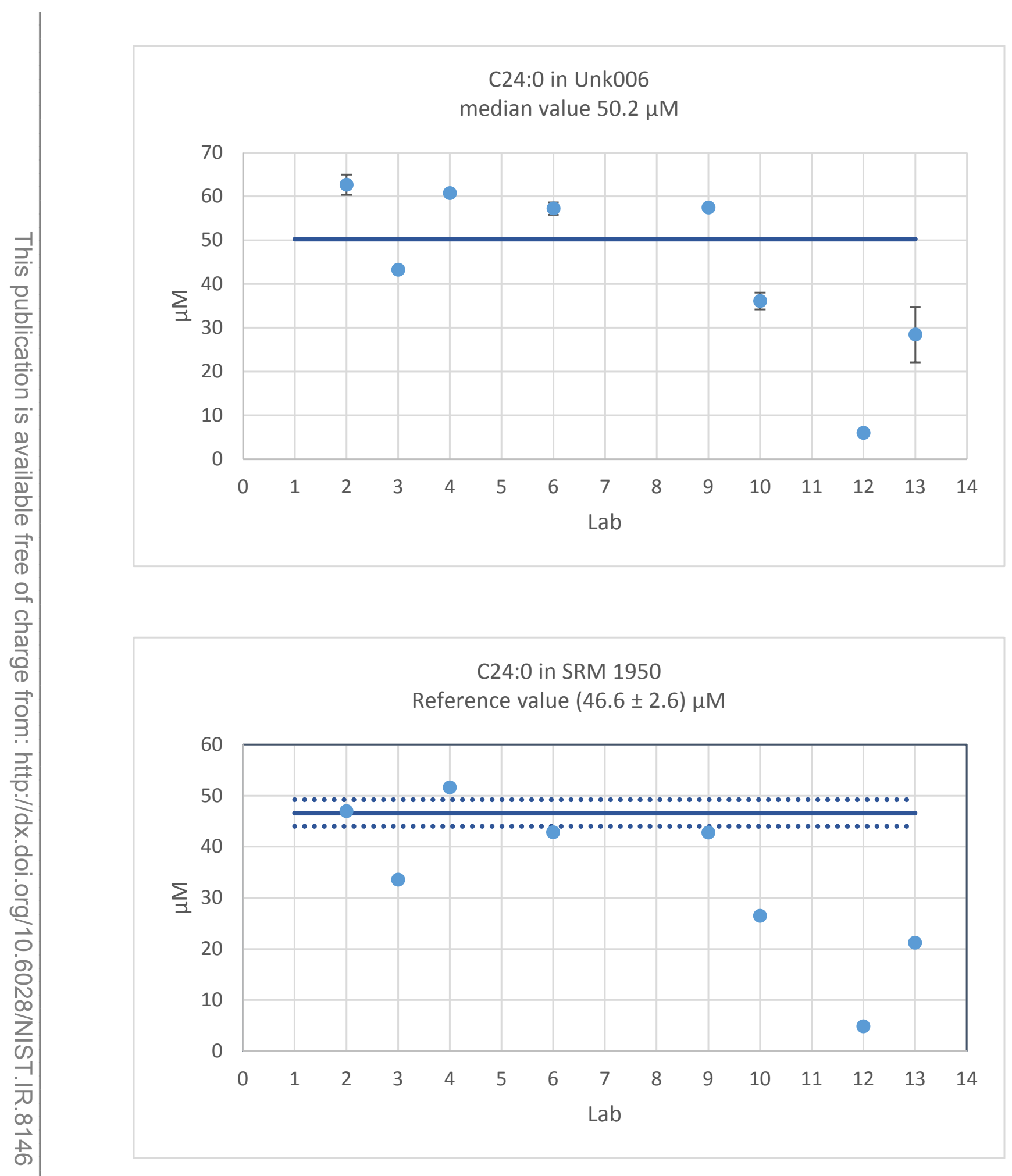



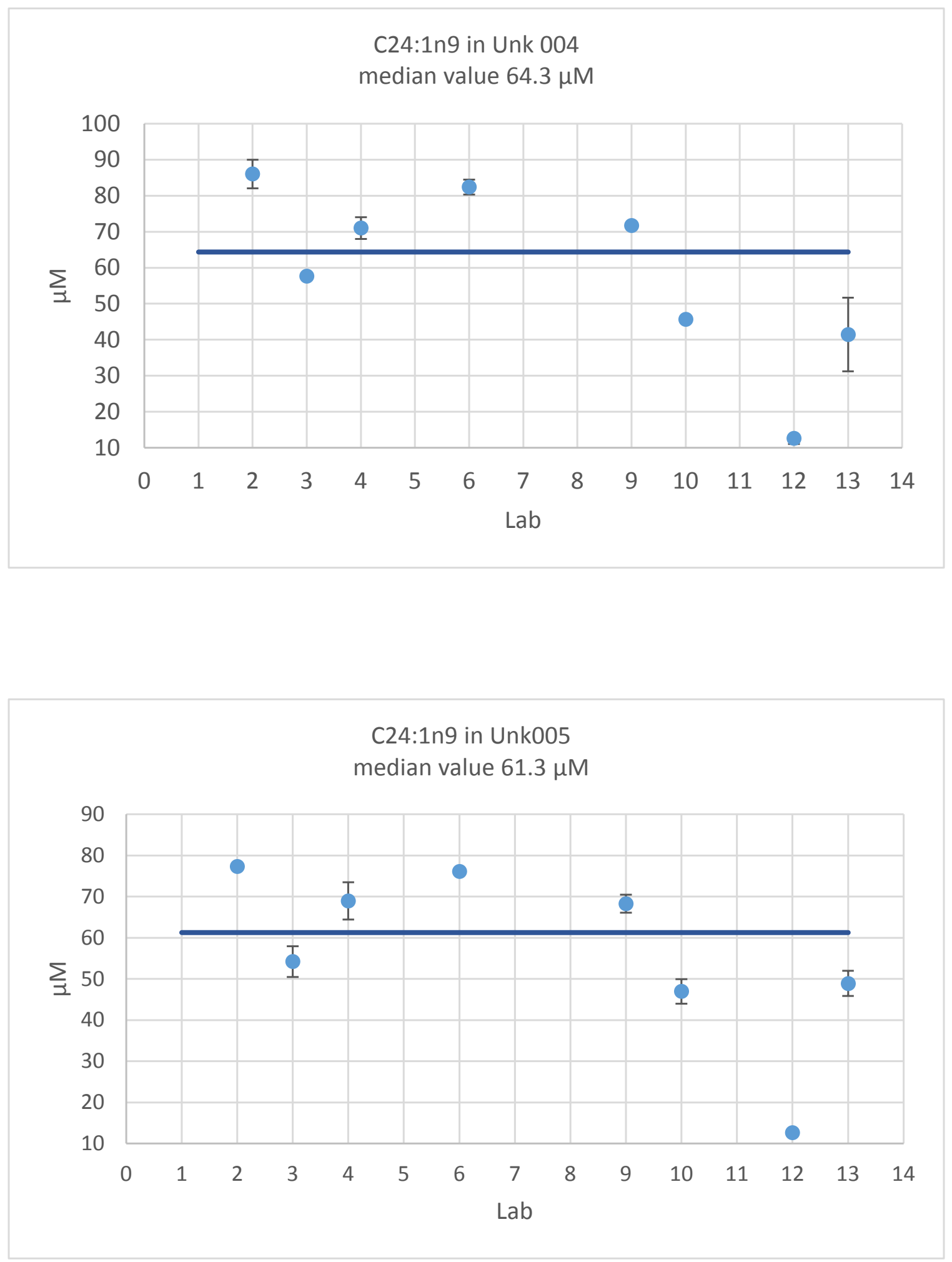

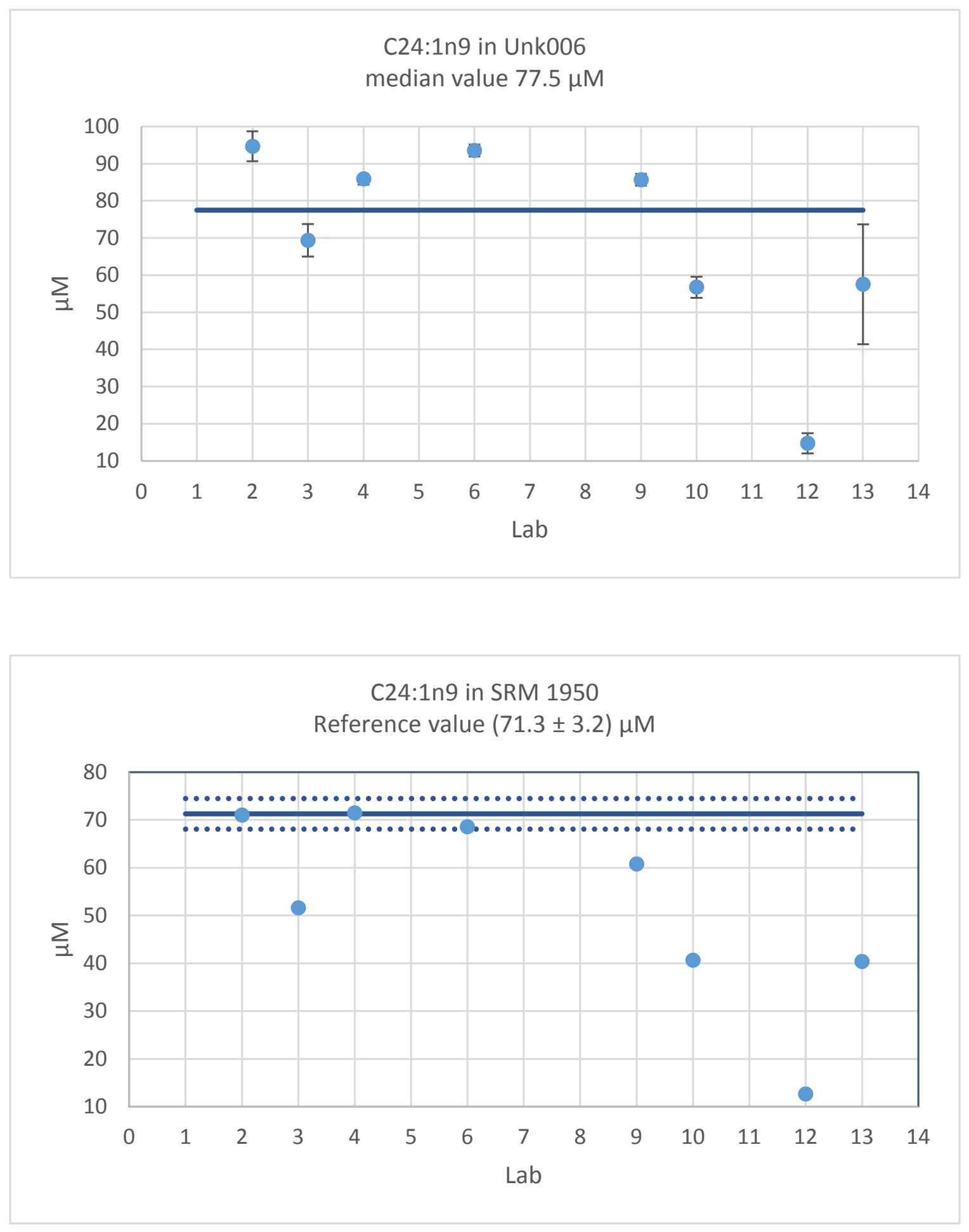


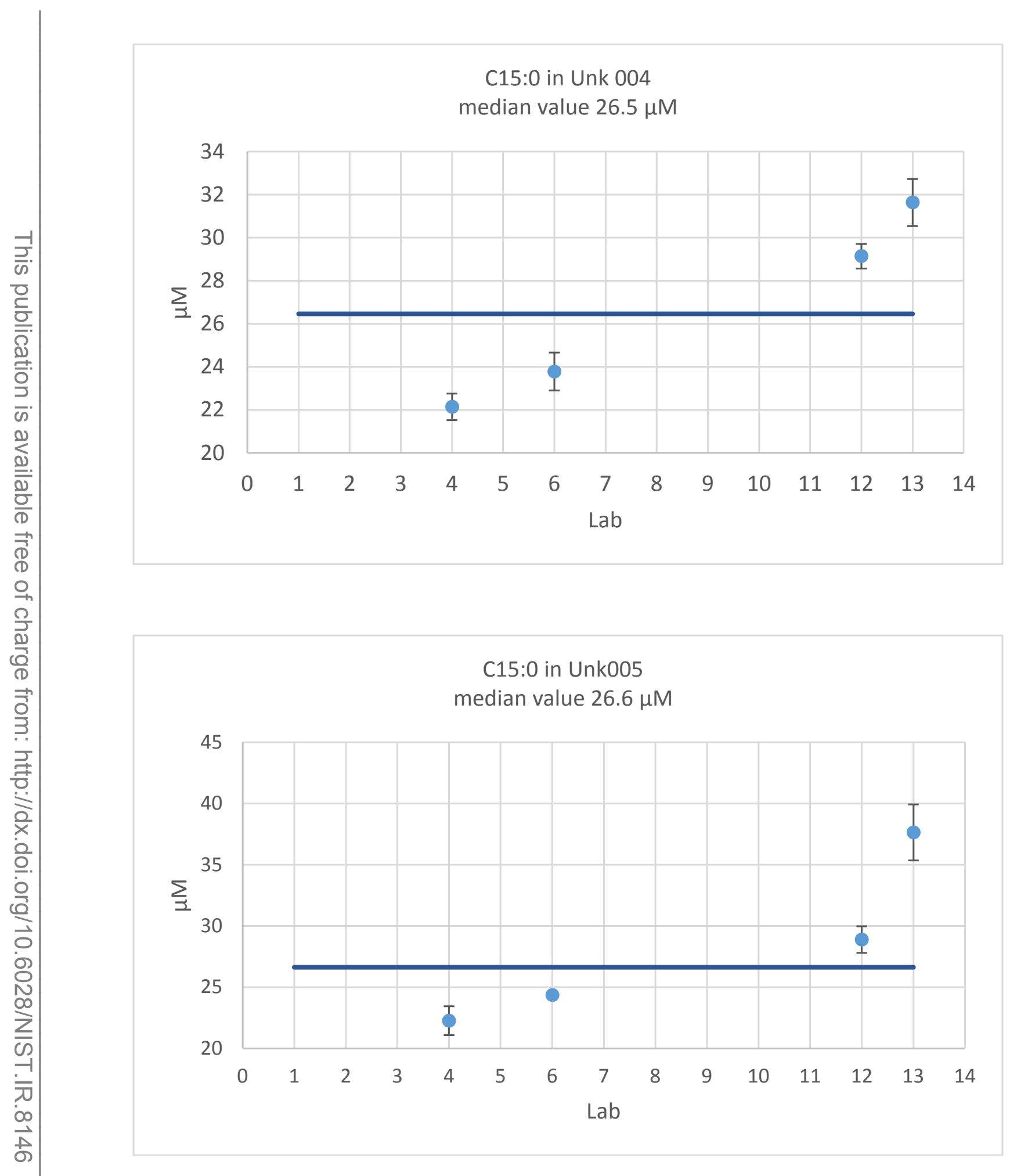




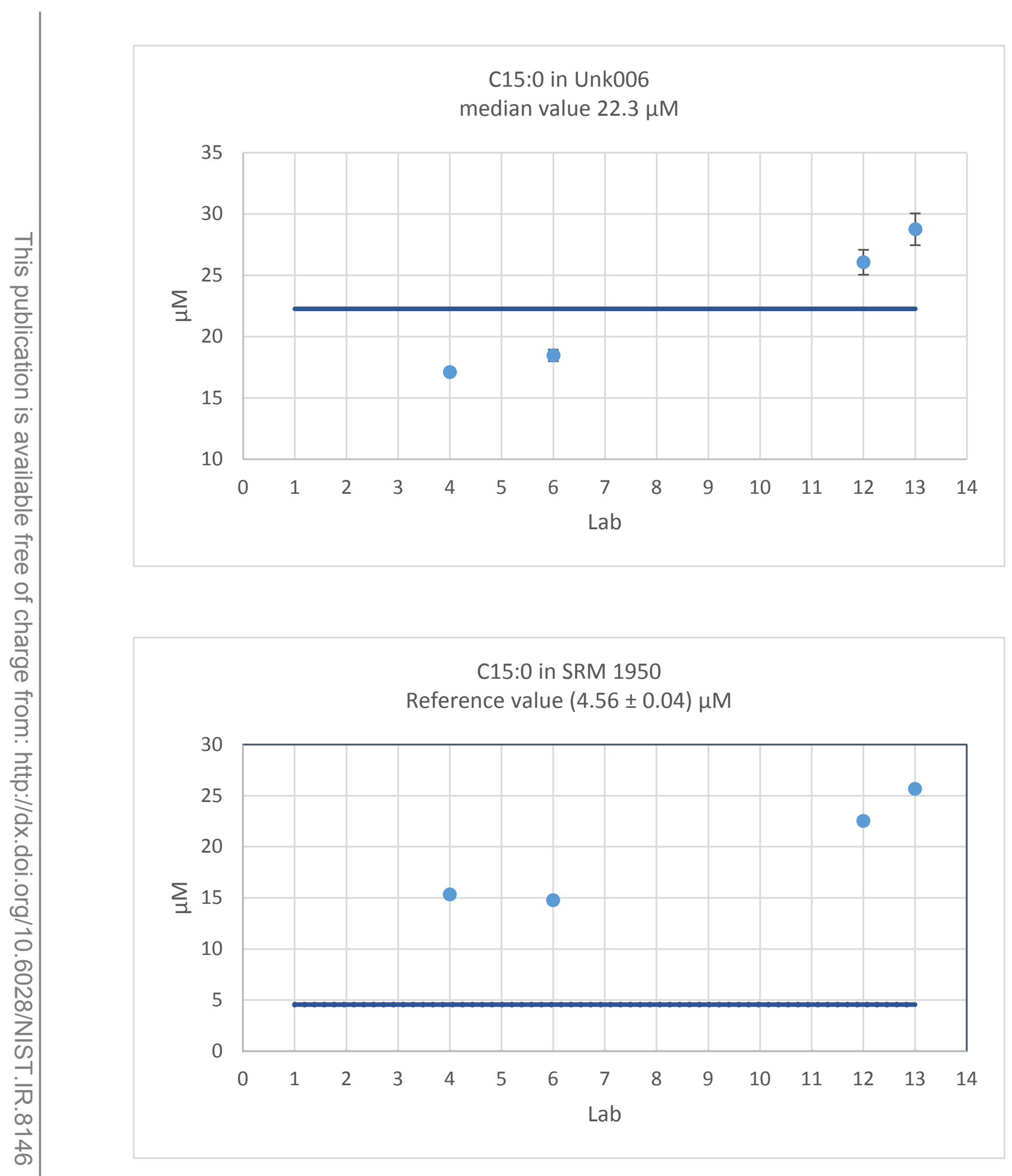




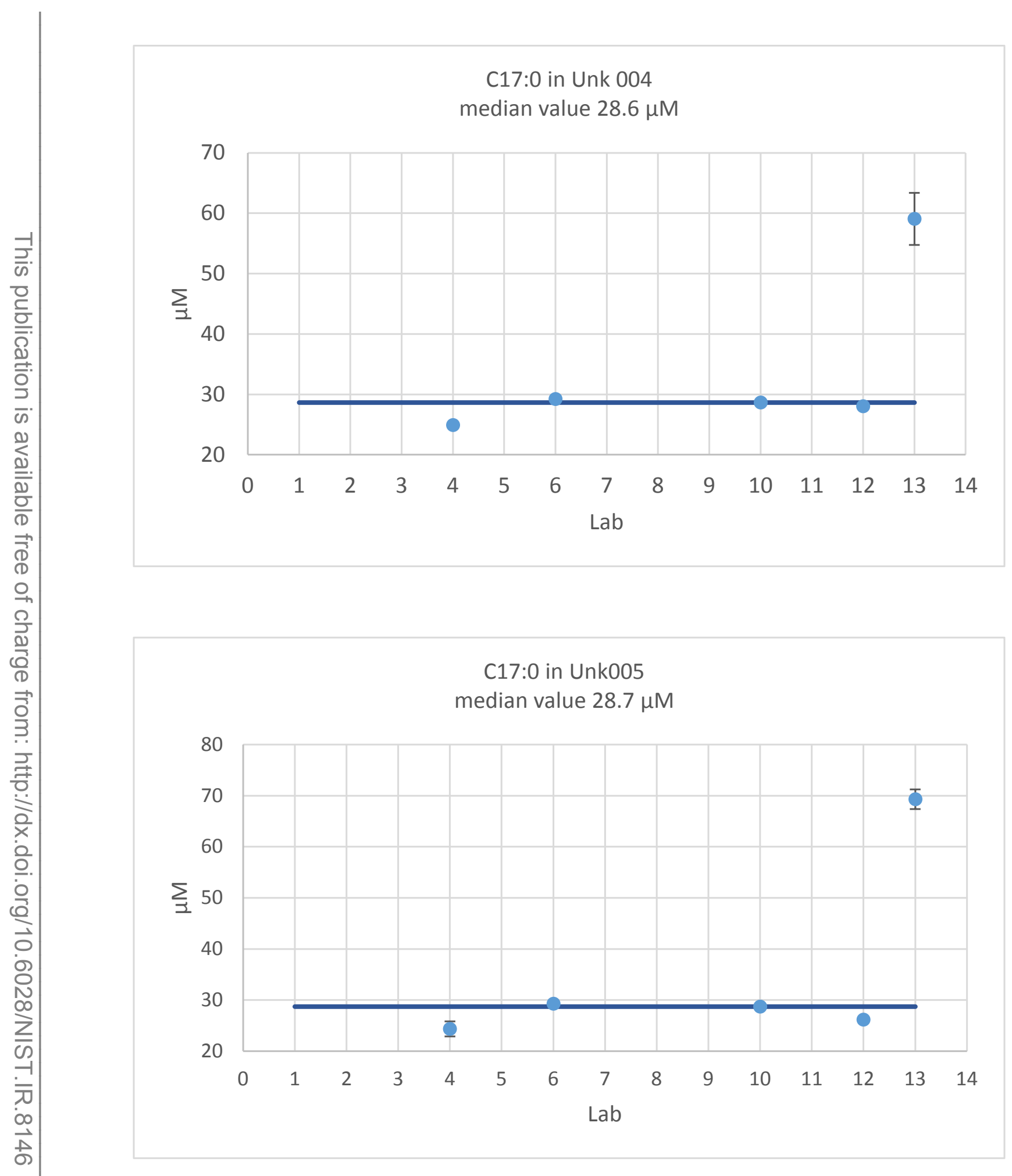




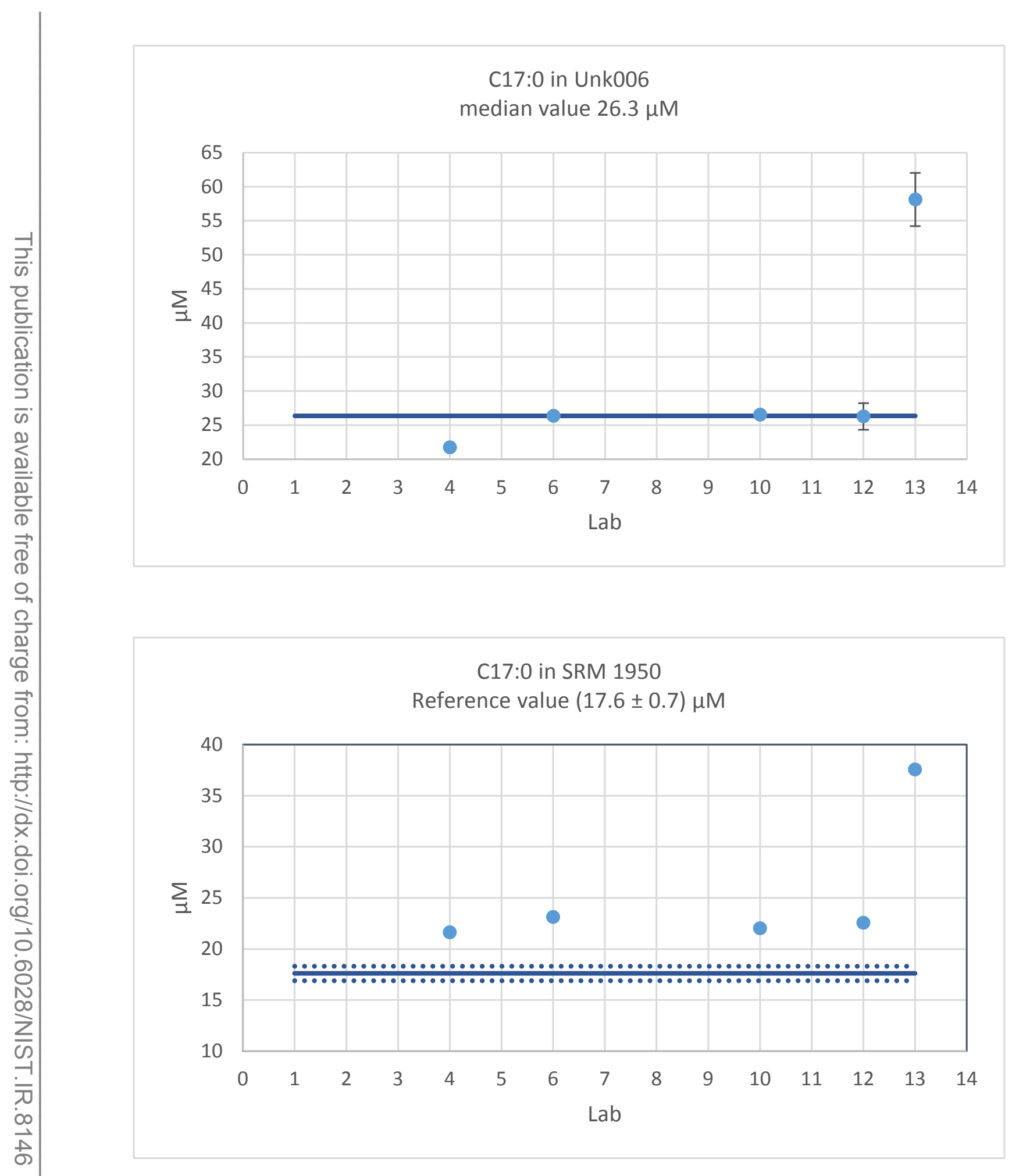




\section{Appendix C.}

Participants in the third interlaboratory analytical comparison study of total fatty acid concentrations in human serum (alphabetical order unrelated to the laboratory numbering in this report)

Bumrungrad Hospital Public Company Limited

Bangkok 10110

THAILAND

Sukon Chalachiva

Centers for Disease Control and Prevention

Clinical Chemistry Branch

Atlanta, GA 30341

Heather Kuiper

Centers for Disease Control and Prevention

Nutritional Biomarkers Branch

Atlanta, GA 30341

Carissa Powers and Rosemary Schleicher

Harvard Chan School of Public Health

Boston, MA

Jeremy Furtado and Matthew Lopes

Matar Pathology

Clinical Chemistry

South Brisbane, Queensland 4101

AUSTRALIA

Matthew Reimer

Mayo Clinic

Biochemical Genetics Laboratory

Rochester, MN 55905

Angela Tauscher

Minnesota Deptartment of Health

Public Health Laboratory

Saint Paul, MN 55155

Shane Olund 
National Institute of Standards and Technology

Chemical Sciences Division

Gaithersburg, MD 20899

Michele Schantz

UCLA

Center for Human Nutrition

Los Angeles, CA 90095

Susanne Henning

University of Michigan

Cancer Center

Ann Arbor, MI 48109

Jianwei Ren and Zora Djuric

University of Utah

Moran Eye Center

Salt Lake City, UT 84106

Aruna Gorusupudi and Paul S. Bernstein

University of Waterloo

Department of Kinesiology

Waterloo, Ontario N2L 5W7

CANADA

Ken Stark

USDA-ARS

Western Human Nutrition Center

Davis, CA 95616

William Keyes and John Newman 